\title{
OBSERVATIONS OF DOUBLE STARS.
}




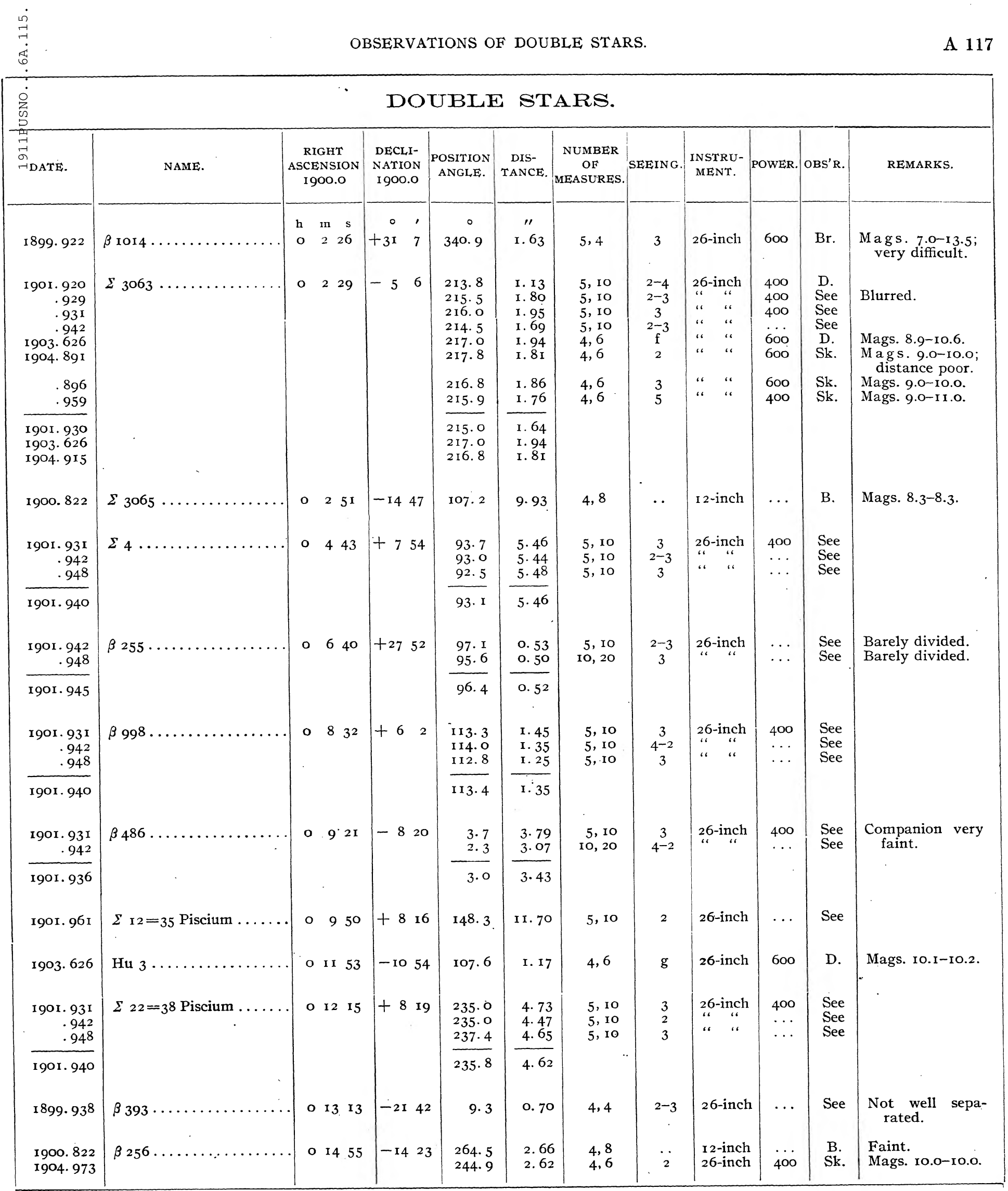




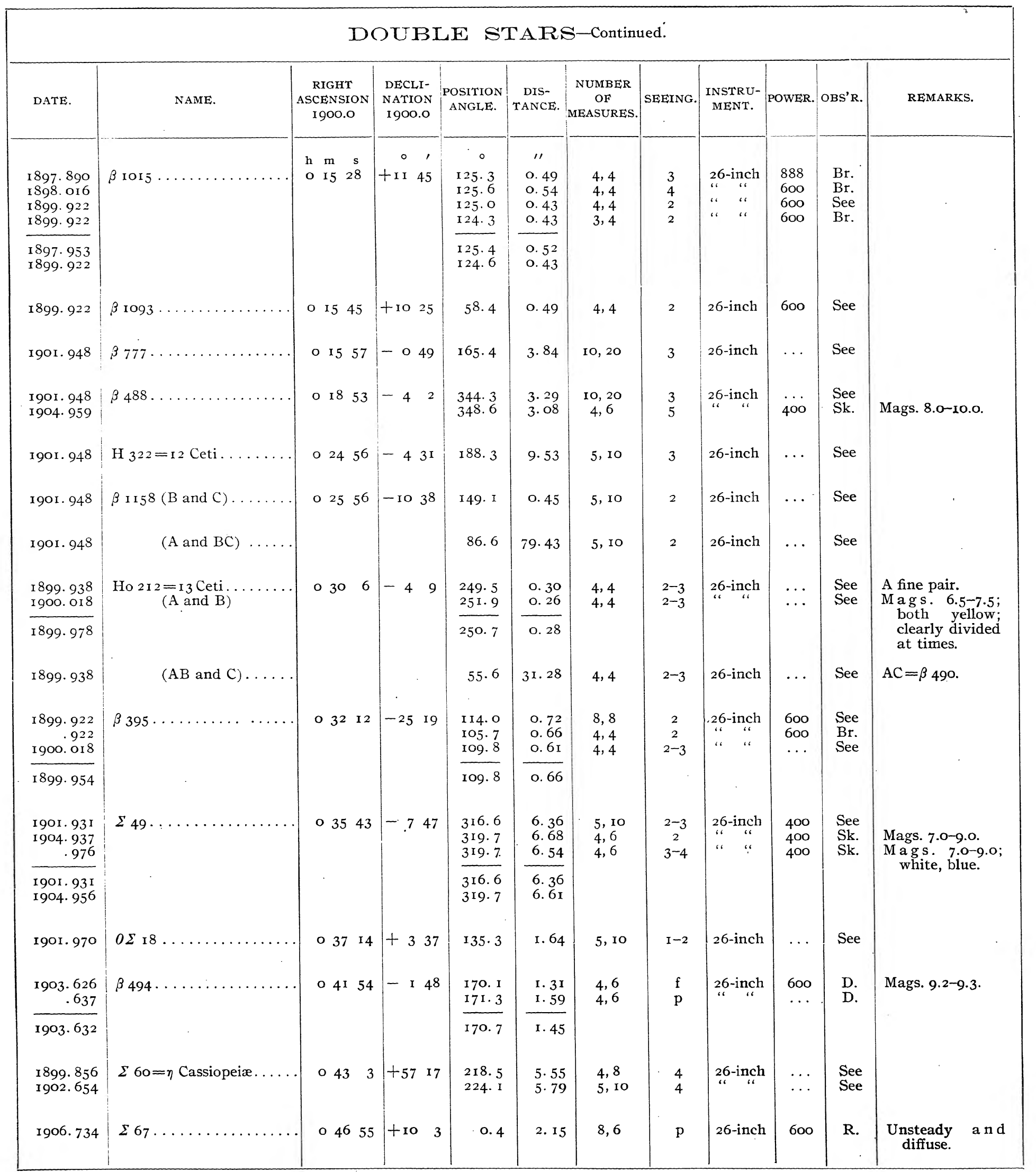




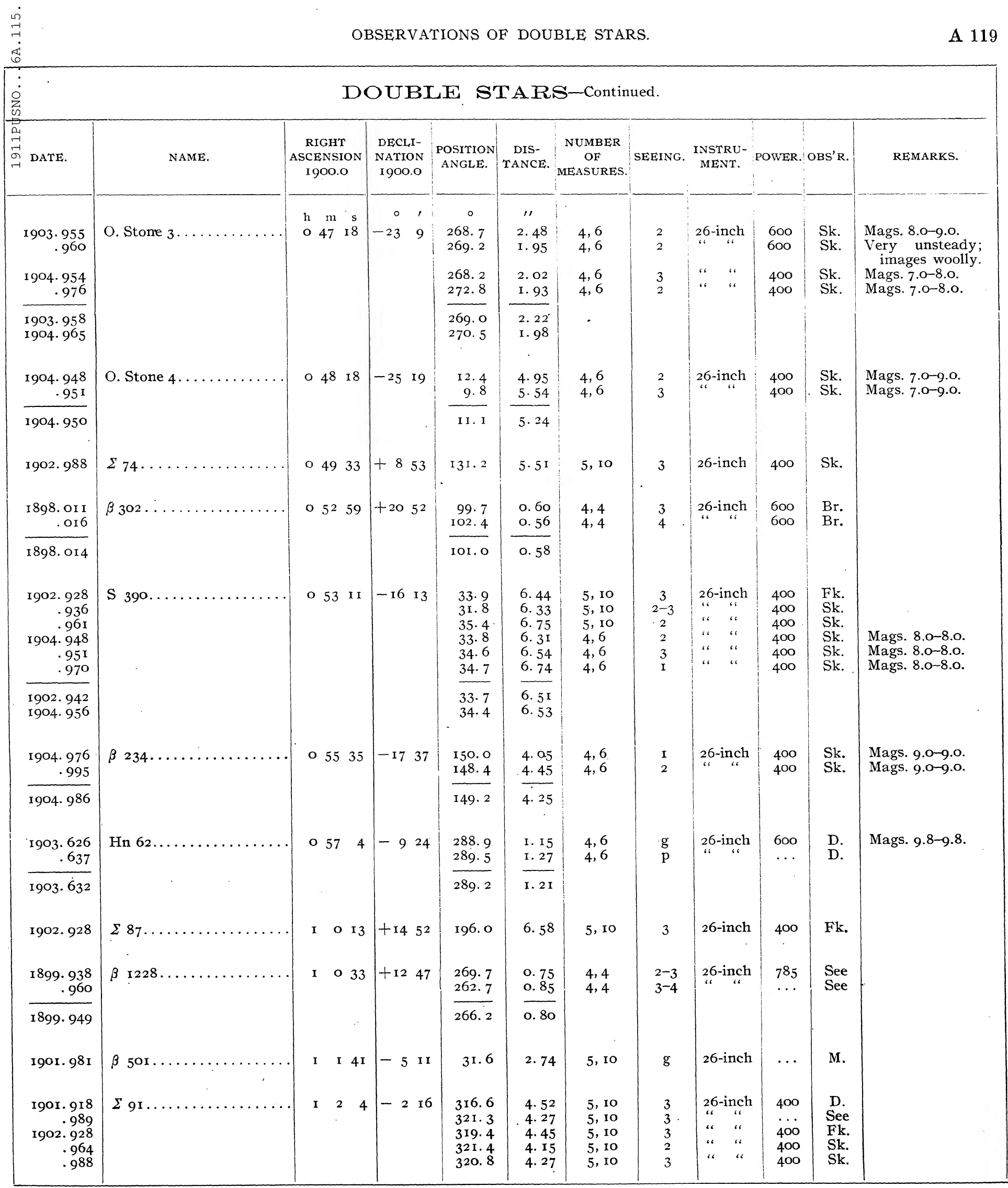


DOUBLE STARS-Continued.

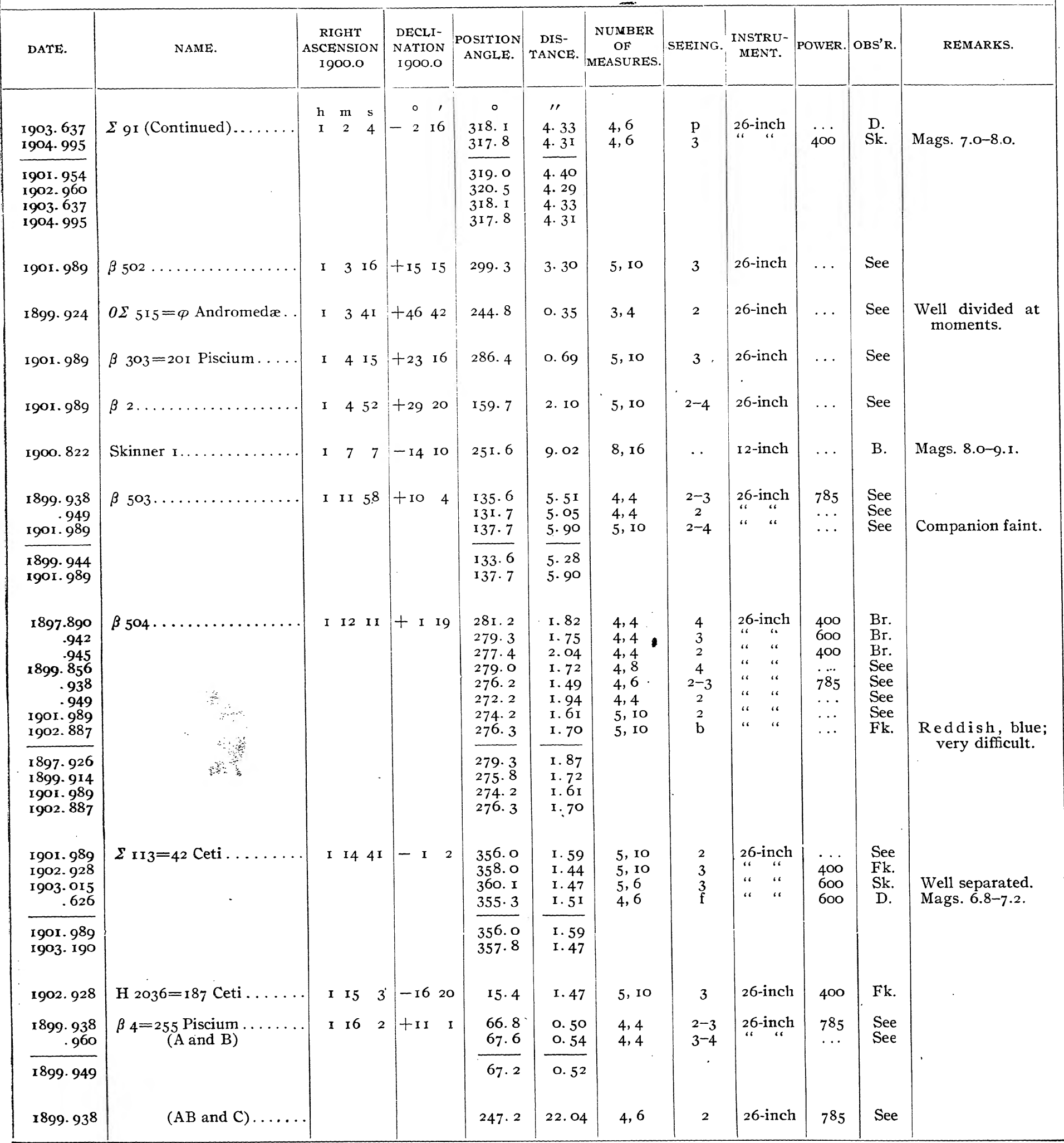




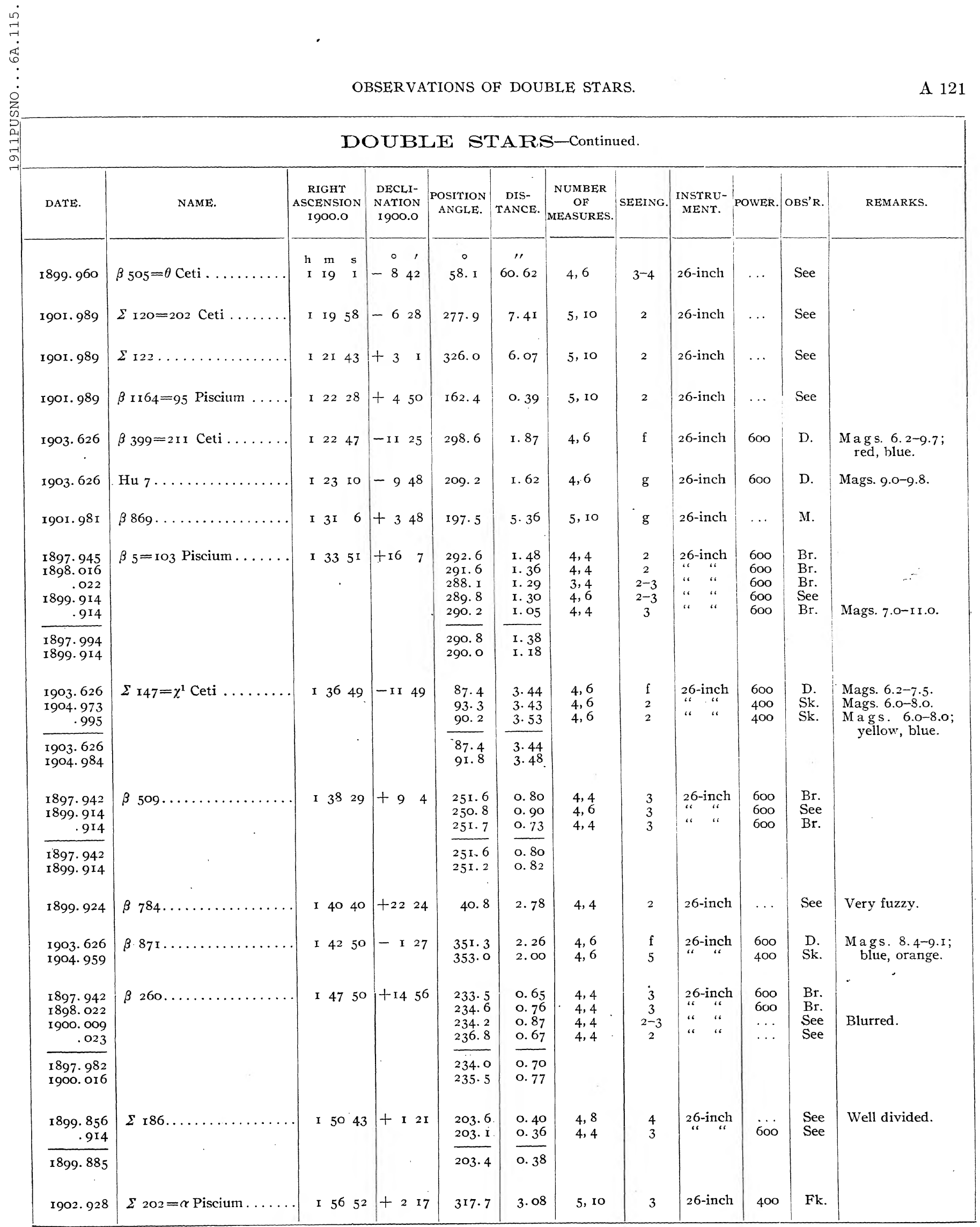




\begin{tabular}{|c|c|c|c|c|c|c|c|c|c|c|c|}
\hline \multicolumn{12}{|c|}{ DOUBLE STARS-Continued. } \\
\hline DATE. & NAME. & $\begin{array}{l}\text { RIGHT } \\
\text { ASCENSION } \\
\text { I9O0.0 }\end{array}$ & $\begin{array}{c}\text { DECLI- } \\
\text { NATION } \\
\text { I9O9O.0 }\end{array}$ & $\begin{array}{c}\text { POSITION } \\
\text { ANGLE. }\end{array}$ & $\begin{array}{c}\text { DIS- } \\
\text { TANCE. }\end{array}$ & $\begin{array}{c}\text { NUMBER } \\
\text { OF } \\
\text { MEASURES. }\end{array}$ & SEEING. & $\begin{array}{l}\text { INSTRU- } \\
\text { MENT. }\end{array}$ & POWER. & OBS'R. & REMARKS. \\
\hline 1899.924 & $\begin{array}{c}0 \Sigma 38=\gamma \text { Andromedx } \\
(\mathrm{B} \text { and } \mathrm{C})\end{array}$ & $\begin{array}{lll}\text { h } & \text { m } & \text { s } \\
\text { I } & 57 & 46\end{array}$ & $\begin{array}{r}0 \\
+4 \mathrm{I} \\
5 \mathrm{I}\end{array}$ & I 12.5 & o. 35 & 4,4 & 2 & 26-inch & $\ldots$ & See & . \\
\hline I 899.924 & $(\mathrm{A}$ and $\mathrm{BC}) \ldots$ & & & 63.6 & $9 \cdot 94$ & 4,6 & 2 & 26-inch & $\cdots$ & See & $\begin{array}{l}\mathrm{AB}=\sum_{2 \mathrm{O} 5}=\text { Hr } \\
\quad \text { III. } 5 .\end{array}$ \\
\hline I901. 989 & $\begin{array}{c}\beta 516 \ldots \ldots \ldots \ldots \ldots \\
\quad .\end{array}$ & 207 & -127 & 286.2 & $0.6 \mathrm{I}$ & 5,10 & 2 & 26-inch & $\ldots$ & See & \\
\hline $\begin{array}{r}1901.989 \\
1903.051 \\
.062 \\
1905.011 \\
\end{array}$ & $\Sigma 218 \ldots \ldots \ldots$ & 2336 & -055 & $\begin{array}{l}249.8 \\
253.0 \\
250.1 \\
249.0\end{array}$ & $\begin{array}{l}5.20 \\
5 \cdot 53 \\
4.99 \\
5.16\end{array}$ & $\begin{array}{l}5,10 \\
4,6 \\
4,6 \\
4,6\end{array}$ & $\begin{array}{c}2 \\
3 \\
3 \\
2-3\end{array}$ & $\begin{array}{cc}26-\text { inch } \\
\text { “" } \\
\text { “ } \\
\text { “ }\end{array}$ & $\begin{array}{l}\ldots \\
400 \\
400 \\
400\end{array}$ & $\begin{array}{l}\text { See } \\
\text { Sk. } \\
\text { Sk. } \\
\text { Sk. }\end{array}$ & Mags. 8.0-9.0. \\
\hline $\begin{array}{l}\text { I901. } 989 \\
1903.056 \\
1905.011\end{array}$ & & & & $\begin{array}{l}249.8 \\
251.6 \\
249.0\end{array}$ & $\begin{array}{l}5.20 \\
5.26 \\
5.16\end{array}$ & & & & & & \\
\hline 1903.626 & Hastings. . . . . . . . . & 2 II 4 & -1842 & $353 \cdot 9$ & 2.42 & 4,6 & f & 26-inch & 600 & D. & $\begin{array}{l}\text { Mags. } 8.1-8.4 \\
\text { green, yellow. }\end{array}$ \\
\hline I901. 989 & $\beta 518=389$ Ceti. . . . . . & $224 \quad 15$ & +97 & 149.9 & I. $8 \mathrm{I}$ & 5 , IO & 2 & 26-inch & $\ldots-$ & See & \\
\hline $\begin{array}{l}1907.91 \mathrm{II} \\
1908.007\end{array}$ & Hammond $\left[\mathrm{B} . \mathrm{D}+4^{\circ} 405\right]$ & 22428 & +426 & $\begin{array}{l}67 \cdot 7 \\
67 \cdot 5\end{array}$ & $\begin{array}{l}3 \cdot 70 \\
3 \cdot 47\end{array}$ & $\begin{array}{l}4,6 \\
4,6\end{array}$ & $\begin{array}{l}\mathrm{g} \\
\mathrm{g}\end{array}$ & $\underset{\text { 26-inch }}{\prime \prime}$ & $\begin{array}{l}600 \\
400\end{array}$ & $\begin{array}{l}\text { Hd. } \\
\text { Hd. }\end{array}$ & $\begin{array}{l}\text { M ags. 9.0-I } 2.8 \\
\text { probably new. }\end{array}$ \\
\hline I907.959 & & . & & $67 \cdot 6$ & $3 \cdot 5^{8}$ & & & . & & & \\
\hline $\begin{array}{l}1900.009 \\
1901.989\end{array}$ & $\beta 519 \ldots \ldots \ldots$ & 22439 & -.243 & $\begin{array}{l}53 \cdot 4 \\
59 \cdot 3\end{array}$ & $\begin{array}{l}0.78 \\
0.88\end{array}$ & $\begin{array}{l}4,4 \\
5,10\end{array}$ & $\begin{array}{c}2-3 \\
2\end{array}$ & 26-inch & $\begin{array}{l}\cdots \\
\cdots\end{array}$ & $\begin{array}{l}\text { See } \\
\text { See }\end{array}$ & \\
\hline 1903.637 & Howe $6 \ldots \ldots \ldots$ & 22645 & $\begin{array}{ll}-8 & 0\end{array}$ & 225.6 & 2.59 & 4,6 & $\mathrm{p}$ & 26-inch & $\ldots$ & D. & \\
\hline $\begin{array}{r}1903.024 \\
.026 \\
.032 \\
.626 \\
1905.011 \\
\end{array}$ & $\Sigma 280 \ldots \ldots \ldots \ldots \ldots$ & $\begin{array}{lll}2 & 29 & 8\end{array}$ & $\begin{array}{ll}-6 & 4\end{array}$ & $\begin{array}{l}166.4 \\
168.7 \\
167.2 \\
166.1 \\
168.2\end{array}$ & $\begin{array}{l}3.66 \\
3.67 \\
3.77 \\
3.86 \\
3.65\end{array}$ & $\begin{array}{l}4,6 \\
4,6 \\
4,6 \\
4,6 \\
4,6\end{array}$ & $\begin{array}{c}3 \\
2-3 \\
2 \\
f \\
3\end{array}$ & 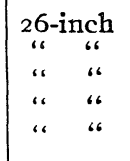 & $\begin{array}{l}600 \\
600 \\
400 \\
600 \\
400\end{array}$ & $\begin{array}{l}\text { Sk. } \\
\text { Sk. } \\
\text { Sk. } \\
\text { D. } \\
\text { Sk. }\end{array}$ & $\begin{array}{l}\text { Mags. 7.7-7.9. } \\
\text { Mags. } 7.0-8.0 .\end{array}$ \\
\hline $\begin{array}{l}\text { 1903. I } 77 \\
1905.011\end{array}$ & & & & $\begin{array}{l}\text { Í́7. I } \\
\text { I68. } 2\end{array}$ & $\begin{array}{l}3.74 \\
3.65\end{array}$ & & & & & & \\
\hline $\begin{array}{l}\text { 1900. } 018 \\
\text { I } 901.9^{89}\end{array}$ & $\beta 520 \ldots \ldots \ldots \ldots$ & 23149 & $\begin{array}{ll}-4 & 0\end{array}$ & $\begin{array}{l}194.0 \\
195.0\end{array}$ & $\begin{array}{l}0.63 \\
0.68\end{array}$ & $\begin{array}{l}4,4 \\
5, \text { Io }\end{array}$ & $\begin{array}{c}2-3 \\
2\end{array}$ & 26-inch & $\begin{array}{l}\cdots \\
\cdots\end{array}$ & $\begin{array}{l}\text { See } \\
\text { See }\end{array}$ & $\begin{array}{c}\text { Barely divided at } \\
\text { best moments. }\end{array}$ \\
\hline $\begin{array}{r}1903.037 \\
.037 \\
.626\end{array}$ & Ho $315 \ldots \ldots \ldots \ldots$ & 2.3353 & $\begin{array}{ll}-2 & I\end{array}$ & $\begin{array}{l}358.3 \\
359.1 \\
358.9\end{array}$ & $\begin{array}{l}\text { I. } 6 \text { I } \\
\text { I. } 39 \\
\text { I. } 67\end{array}$ & $\begin{array}{l}4,6 \\
4,6 \\
4,6\end{array}$ & $\begin{array}{l}3 \\
\mathrm{p} \\
\mathrm{f}\end{array}$ & \begin{tabular}{|c|} 
26-inch \\
“" \\
$“ " 1$
\end{tabular} & $\begin{array}{l}600 \\
600 \\
600\end{array}$ & $\begin{array}{l}\text { Sk. } \\
\text { Fk. } \\
\text { D. }\end{array}$ & $\begin{array}{l}\text { Mags. } 9.4-9.6 \\
\text { blue, yellow. }\end{array}$ \\
\hline 1903.233 & & & & 358.8 & I. 56 & & & & & & \\
\hline $\begin{array}{r}\mathrm{I} 902.96 \mathrm{I} \\
.977 \\
\mathrm{I} 903.05 \mathrm{I} \\
.062 \\
.637 \\
\end{array}$ & $\Sigma 295=84$ Ceti... & $\begin{array}{lll}2 & 36 & 6\end{array}$ & 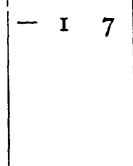 & $\begin{array}{l}322.6 \\
319.5 \\
318.7 \\
320.8 \\
323.6 \\
\end{array}$ & $\begin{array}{l}4.75 \\
4.67 \\
4.55 \\
4.51 \\
4.31 \\
\end{array}$ & $\begin{array}{l}5,10 \\
5,10 \\
4,6 \\
4,6 \\
4,6\end{array}$ & $\begin{array}{l}\mathrm{p} \\
3 \\
3 \\
3 \\
\mathrm{~b}\end{array}$ & $\begin{array}{cc}26-i n c h \\
، \\
، & 6 \\
، & ، \\
& 4\end{array}$ & $\begin{array}{l}400 \\
400 \\
400 \\
400 \\
\cdots\end{array}$ & $\begin{array}{l}\text { Fk. } \\
\text { Sk. } \\
\text { Sk. } \\
\text { Sk. } \\
\text { D. }\end{array}$ & \\
\hline $1903 \cdot 138$ & & & & 321.0 & $4 \cdot 5^{6}$ & & & & & & \\
\hline
\end{tabular}


DOUBIE STARS-Continued.

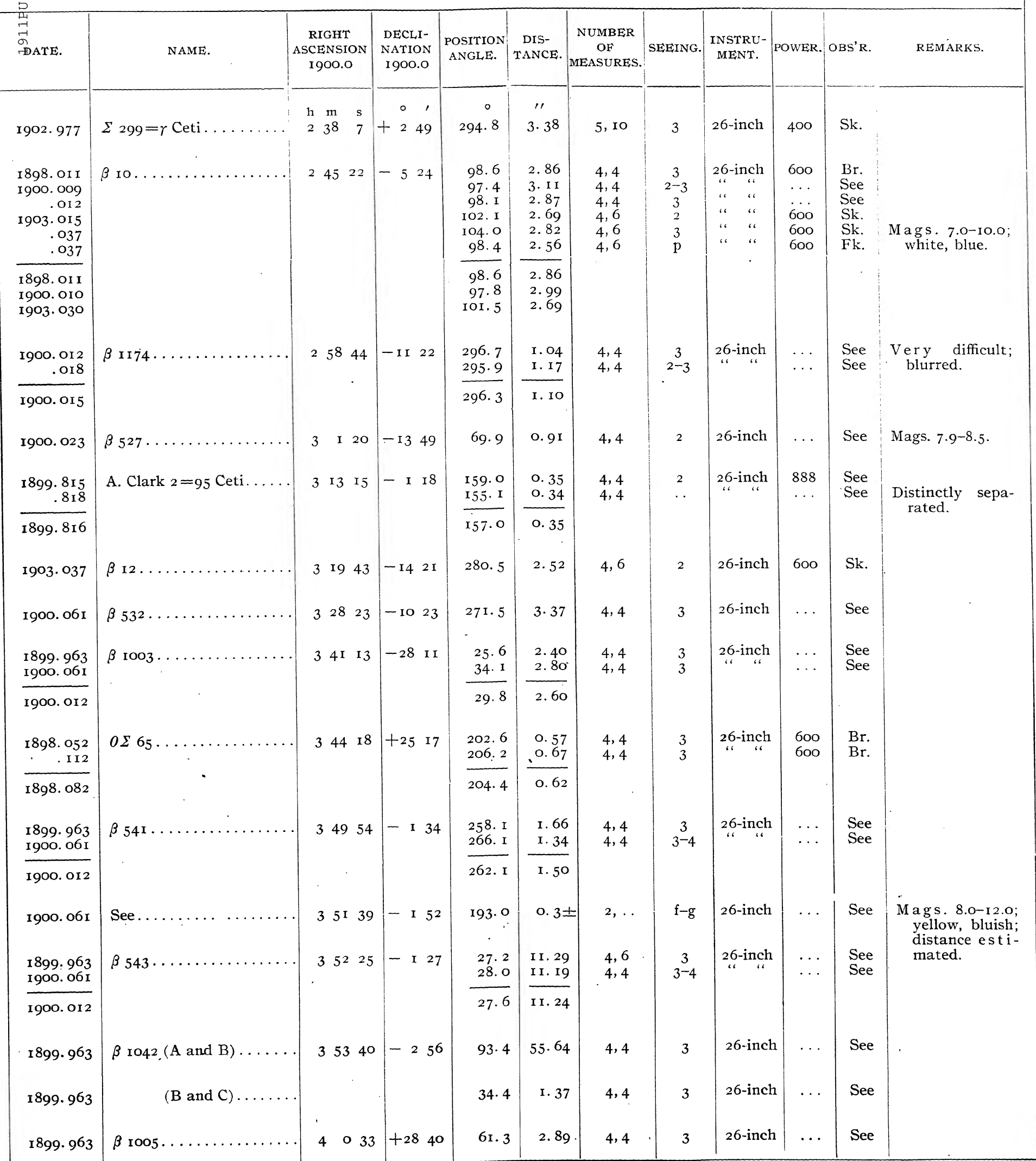




\begin{tabular}{|c|c|c|c|c|c|c|c|c|c|c|c|}
\hline \multicolumn{12}{|c|}{ DOUBLE STARS-Continued. } \\
\hline DATE. & NAME. & $\begin{array}{c}\text { RIGHT } \\
\text { ASCENSION } \\
\text { I } 900.0\end{array}$ & $\begin{array}{l}\text { DECLI- } \\
\text { NATION } \\
1900.0\end{array}$ & $\begin{array}{l}\text { POSITION } \\
\text { ANGLE. }\end{array}$ & $\begin{array}{c}\text { DIS- } \\
\text { TANCE. }\end{array}$ & $\begin{array}{c}\text { NUMBER } \\
\text { OF } \\
\text { MEASURES. }\end{array}$ & SEEING. & $\begin{array}{c}\text { INSTRU- } \\
\text { MENT. }\end{array}$ & POWER. & OBS'R. & REMARKS. \\
\hline 1898.126 & $0 \Sigma 531 \ldots \ldots \ldots \ldots$ & $\begin{array}{ccr}\mathrm{h} & \mathrm{m} & \mathrm{s} \\
4 & \mathrm{O} & 54\end{array}$ & $\begin{array}{r}0 \\
+3749\end{array}$ & $\stackrel{\circ}{\circ} \stackrel{-0}{128.0}$ & $\begin{array}{l}\prime \prime \\
\text { I. } 97\end{array}$ & 4,4 & 2 & 26-inch & 600 & $\mathrm{Br}$. & Unsteady. \\
\hline $\begin{array}{l}1907.930 \\
1908.007 \\
\end{array}$ & Hu 8 I $_{7} \ldots \ldots \ldots \ldots$ & 4 II 45 & +3223 & $\begin{array}{l}249.5 \\
248.1 \\
\end{array}$ & $\begin{array}{l}3 \cdot 32 \\
3 \cdot 34 \\
\end{array}$ & $\begin{array}{l}4,6 \\
5,6\end{array}$ & $\begin{array}{l}\mathrm{g} \\
\mathrm{g}\end{array}$ & "6-inch & $\begin{array}{l}600 \\
400\end{array}$ & $\begin{array}{l}\text { Hd. } \\
\text { Hd. }\end{array}$ & Mags. 9.0-I 2.5. \\
\hline $1907 \cdot 968$ & & & & 248.8 & $3 \cdot 33$ & & & & & & \\
\hline $\begin{array}{r}1903.051 \\
.062 \\
\end{array}$ & $\Sigma \Sigma_{536} \ldots \ldots$ & $\begin{array}{lll}4 & 17 & 13\end{array}$ & -455 & $\begin{array}{l}165.3 \\
166.4 \\
\end{array}$ & $\begin{array}{l}2.01 \\
2.00 \\
\end{array}$ & $\begin{array}{l}4,6 \\
4,6\end{array}$ & $\begin{array}{c}2 \\
2-3\end{array}$ & "26-inch & $\begin{array}{l}600 \\
600\end{array}$ & $\begin{array}{l}\text { Sk. } \\
\text { Sk. }\end{array}$ & \\
\hline 1903.056 & & & & 165.8 & 2.00 & & & & & & \\
\hline I900. 06I & $\beta$ 744=299 Eridani $\ldots$ & 41722 & $-255^{8}$ & 316.8 & o. 89 & 4,4 & $3-4$ & 26-inch & $\cdots$ & See & \\
\hline I902. 96I & $\Sigma 535=230$ Tauri..... & $\begin{array}{llll}4 & 17 & 45\end{array}$ & t 119 & $327 \cdot \mathrm{I}$ & I. 59 & 5, 10 & $\mathrm{p}$ & 26-inch & 400 & Fk. & \\
\hline 1899.974 & $\beta$ 1235 $\ldots \ldots \ldots \ldots$ & $\begin{array}{llll}4 & 18 & 31\end{array}$ & +2231 & 240.6 & $0.3^{8}$ & 4,4 & 4 & 26-inch & $\ldots$ & See & Barely divided. \\
\hline I900. $06 \mathrm{I}$ & $\beta$ II $86=248$ Tauri. . . . . & 42158 & +10 59 & 176.9 & o. 57 & 4,4 & $3-4$ & 26-inch & $\cdots$ & See & \\
\hline 1900.061 & $\Sigma 554=80$ Tauri $\ldots \ldots$ & $\begin{array}{lll}4 & 24 & 26\end{array}$ & +1525 & $44 \cdot 2$ & 0.60 & 4,4 & $3-4$ & 26-inch & $\cdots$ & See & Mags. 6-I0.5. \\
\hline 1903.081 & $\Sigma 590=55$ Eridani $\ldots \ldots$ & 43847 & -859 & 317.6 & $9 \cdot 38$ & 4,6 & 2 & 26-inch & 600 & Sk. & \\
\hline $\begin{array}{r}1899.755 \\
.963 \\
.976 \\
1901.080 \\
1902.222 \\
\end{array}$ & $\beta 883(\mathrm{~A}$ and $\mathrm{B}) \ldots$ & 44540 & tio 54 & $\begin{array}{l}58.7 \\
53.7 \\
53.6 \\
70.2 \\
68.8\end{array}$ & $\begin{array}{l}\text { o. } 38 \\
0.27 \\
0.28 \\
\text { o. } 23 \\
\text { o. } 32 \\
\end{array}$ & $\begin{array}{l}4,8 \\
4,4 \\
4,4 \\
4,8 \\
5, \text { ro }\end{array}$ & $\begin{array}{c}4 \\
3-4 \\
4 \\
2-3 \\
2-3\end{array}$ & 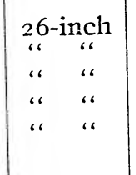 & $\begin{array}{l}\cdots \\
\cdots \\
888 \\
600\end{array}$ & $\begin{array}{l}\text { See } \\
\text { See } \\
\text { See } \\
\text { See } \\
\text { See }\end{array}$ & $\begin{array}{l}\text { Divided at best } \\
\text { moments. } \\
\text { Nicely divided. }\end{array}$ \\
\hline $\begin{array}{l}1899.898 \\
1901.080 \\
1902.222\end{array}$ & & . & & $\begin{array}{l}55 \cdot 3 \\
70.2 \\
68.8\end{array}$ & $\begin{array}{l}\text { o. } 31 \\
0.23 \\
0.32\end{array}$ & & & & & & \\
\hline $\begin{array}{r}1899.963 \\
.976 \\
1902.222\end{array}$ & $(A B$ and $C) \ldots \ldots$ & & & $\begin{array}{l}154.3 \\
154.9 \\
155.6 \\
\end{array}$ & $\begin{array}{l}17.71 \\
17.84 \\
17.78 \\
\end{array}$ & $\begin{array}{l}4,4 \\
4,4 \\
5, \text { ro }\end{array}$ & $\begin{array}{c}3-4 \\
4 \\
2-3\end{array}$ & 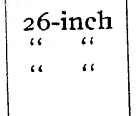 & $\begin{array}{l}\ldots \\
\cdots \\
6 \mathrm{oo}\end{array}$ & $\begin{array}{l}\text { See } \\
\text { See } \\
\text { See }\end{array}$ & \\
\hline $\begin{array}{l}1899.970 \\
1902.222\end{array}$ & . & & & $\begin{array}{l}\text { I54. } 6 \\
\text { I55. } 6\end{array}$ & $\begin{array}{l}\mathrm{I} 7 \cdot 78 \\
\mathrm{I} 7 \cdot 78\end{array}$ & & & & & & \\
\hline I $899 \cdot 755$ & $\beta 552=$ I I Orionis.... & 446 II & $+\mathrm{I} 329$ & $202 \cdot 3$ & 0.60 & 4,4 & $\dot{4}$ & 26-inch & $\cdots$ & See & $\begin{array}{l}\text { M a g s i 7.o-ro. } 5 \\
\text { obs. doubtful. }\end{array}$ \\
\hline .963 & & & & 200.4 & o. 43 & 4,4 & $3-4$ & “" “ & $\cdots$ & See & $\begin{array}{l}\text { Divided at best } \\
\text { moments. }\end{array}$ \\
\hline $\begin{array}{r}.976 \\
1902.222 \\
\end{array}$ & . & & & $\begin{array}{l}202.8 \\
210.3\end{array}$ & $\begin{array}{l}\text { o. } 36 \\
\text { o. } 34 \\
\end{array}$ & $\begin{array}{l}4,4 \\
4, \text { Io }\end{array}$ & $\begin{array}{c}4 \\
2-3\end{array}$ & “" $" ،$ & 600 & $\begin{array}{l}\text { See } \\
\text { See }\end{array}$ & $\begin{array}{l}\text { Divided at best } \\
\text { moments. }\end{array}$ \\
\hline $\begin{array}{l}1899.898 \\
1902.222\end{array}$ & $\cdot$ & & & $\begin{array}{l}201.8 \\
210.3\end{array}$ & $\begin{array}{l}\text { o. } 46 \\
\text { o. } 34\end{array}$ & & & & & & \\
\hline 1901.080 & $\beta 1237 \ldots \ldots \ldots \ldots$ & 44740 & +2323 & 60.0 & $4 \cdot 3^{2}$ & 4,8 & $2-3$ & 26-inch & 600 & See & \\
\hline 1898. II & $0 \Sigma 90 \ldots \ldots \ldots \ldots \ldots$ & 44930 & +826 & $34 \mathrm{I} \cdot 4$ & 2. OI & 3,4 & 2 & 26-inch & 600 & $\mathrm{Br}$. & \\
\hline $\begin{array}{l}\text { I898. I I5 } \\
1899.97 \text { I }\end{array}$ & $\beta 314=3$ Leporis. $\ldots$ & $\begin{array}{lll}4 & 54 & 33\end{array}$ & -1632 & $\begin{array}{l}326.2 \\
3 \text { 10. } 5\end{array}$ & $\begin{array}{l}0.86 \\
0.96\end{array}$ & $\begin{array}{l}4,4 \\
4,4\end{array}$ & $\begin{array}{l}4 \\
2\end{array}$ & ${ }_{4 "}^{26-i n c h}$ & 600 & $\begin{array}{l}\text { Br. } \\
\text { See }\end{array}$ & Mags. 7.0-10.0. \\
\hline
\end{tabular}




\begin{tabular}{|c|c|c|c|c|c|c|c|c|c|c|c|c|}
\hline \multicolumn{12}{|c|}{ OBSERVATIONS OF DOUBLE STARS. } & \multirow[t]{2}{*}{ A 125} \\
\hline \multicolumn{12}{|c|}{ DOUBLE STARS-Continued. } & \\
\hline DATE. & NAME. & $\begin{array}{r}\text { RIGHT } \\
\text { ASCENSI } \\
\text { I } 900.0\end{array}$ & $\begin{array}{l}\text { IT } \\
\text { SION } \\
.0\end{array}$ & \begin{tabular}{|c|} 
DECLI- \\
NATION \\
I9OO.O
\end{tabular} & $\begin{array}{c}\text { POSITION } \\
\text { ANGLE. }\end{array}$ & $\begin{array}{c}\text { DIS- } \\
\text { TANCE. }\end{array}$ & $\begin{array}{c}\text { NUMBER } \\
\text { OF } \\
\text { MEASURES. }\end{array}$ & SEEING. & $\begin{array}{l}\text { INSTRU- } \\
\text { MENT. }\end{array}$ & POWER. & OBS'R. & REMARKS. \\
\hline 1898.189 & $0 \Sigma 93 \ldots \ldots \ldots \ldots \ldots$ & $\begin{array}{ll}\mathrm{h} & \mathrm{m} \\
4 & 55\end{array}$ & $\begin{array}{r}s \\
\text { II }\end{array}$ & $\begin{array}{r}0 \\
+457\end{array}$ & $\begin{array}{c}\circ \\
51.8\end{array}$ & o. $5^{6}$ & 4,4 & 2 & 26-inch & 888 & Br. & \\
\hline $1903.08 \mathrm{I}$ & $\Sigma 636 \ldots \ldots \ldots \ldots \ldots$ & $45^{8}$ & 35 & $-84^{8}$ & IOO. 3 & $3 \cdot 73$ & 4,6 & $2-3$ & 26-inch & 600 & Sk. & \\
\hline I $899 \cdot 976$ & $\beta 1047 \underset{(A \text { and } B)}{=47}$ Aurigæ...... & 53 & 28 & +2754 & $27 \cdot 4$ & хx. 66 & 4,4 & 4 & 26-inch & $\ldots$ & See & \\
\hline $\begin{array}{r}1898 . \text { I1 } 5 \\
1899.963 \\
.971 \\
\end{array}$ & $\beta 885 \ldots \ldots$ & $5 \quad 5$ & 54 & - I 53 & $\begin{array}{l}194.8 \\
\text { I91. } 7 \\
\text { I } 88.4\end{array}$ & $\begin{array}{l}0.70 \\
0.77 \\
0.82 \\
\end{array}$ & $\begin{array}{l}4,4 \\
4,4 \\
4,4\end{array}$ & $\begin{array}{c}4 \\
3-4 \\
2\end{array}$ & 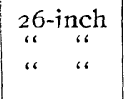 & $\begin{array}{l}600 \\
\cdots \\
\cdots\end{array}$ & $\begin{array}{l}\text { Br. } \\
\text { See } \\
\text { See }\end{array}$ & \\
\hline $\begin{array}{l}\text { I898. I15 } \\
\text { 1899. } 967\end{array}$ & . & & & & $\begin{array}{l}\text { 194. } 8 \\
\text { 190.0 }\end{array}$ & $\begin{array}{l}0.70 \\
0.80\end{array}$ & & & & & & \\
\hline .169 & $\Sigma 66 \mathrm{I}=\kappa$ Leporis....... & 58 & 837 & $\begin{array}{ll}-13 & 4\end{array}$ & 360.0 & 2. 84 & 4,6 & $2-3$ & $\begin{array}{c}26 \text {-inch } \\
\text { ، }\end{array}$ & 600 & Sk. & $\begin{array}{l}\text { Mags. } 5.0-8.0 ; \\
\text { whi te, blue; } \\
\text { measures diff- } \\
\text { cult. } \\
\text { Mags. 5.0 -7.0. }\end{array}$ \\
\hline 1903.125 & & & & & $35^{8.0}$ & 2.60 & & & & & & \\
\hline $\begin{array}{l}\text { I898. I15 } \\
\text { I899.976 }\end{array}$ & $\beta$ 3I $8 \ldots \ldots \ldots \ldots \ldots$ & $5 \mathrm{YI}$ & I 15 & -336 & $\begin{array}{l}238.3 \\
234.4\end{array}$ & $\begin{array}{l}0.57 \\
0.62\end{array}$ & $\begin{array}{r}4,4 \\
4,4\end{array}$ & $\begin{array}{c}4 \\
3-4\end{array}$ & 26-inch & $\begin{array}{l}888 \\
\cdots\end{array}$ & $\begin{array}{l}\text { Br. } \\
\text { See }\end{array}$ & \\
\hline $\begin{array}{l}\mathrm{I} 899.976 \\
\mathrm{I} 900.042 \\
\end{array}$ & $\Sigma 728=32$ Orionis........ & 525 & 526 & $+55^{2}$ & $\begin{array}{l}149.8 \\
151.5\end{array}$ & $\begin{array}{l}0.26 \\
0.30\end{array}$ & $\begin{array}{l}4,4 \\
4,4\end{array}$ & $\begin{array}{c}3-4 \\
4\end{array}$ & 26-inch & $\begin{array}{l}\cdots \\
\cdots\end{array}$ & $\begin{array}{l}\text { See } \\
\text { See }\end{array}$ & \\
\hline 1900.009 & & & & & I50.6 & 0.28 & & & & & & \\
\hline 1903. 109 & $\Sigma 729=33$ Orionis. . . . . . & 526 & 60 & +313 & 22.4 & I. 72 & 4,8 & $\mathrm{~g}$ & 12-inch & 335 & $\mathrm{~K}$. & \\
\hline 1903. 109 & $\Sigma 738=\lambda$ Orionis $\ldots \ldots \ldots$ & 529 & 938 & $+95^{I}$ & 40.5 & 4. 10 & 4,8 & f & r2-inch & 335 & $\mathrm{~K}$. & \\
\hline 1903.037 & $\Sigma 774=\underset{(\mathrm{A} \text { and } \mathrm{B})}{\zeta}$ Orionis $\cdots \cdots$ & 535 & 543 & $\begin{array}{ll}-2 & 0\end{array}$ & $\mathrm{I}_{5} 6.6$ & 2.48 & 4,6 & $\mathrm{p}$ & 26-inch & 600 & Fk. & \\
\hline 1898. I 12 & $o \Sigma \Sigma_{1} \ldots \ldots \ldots \ldots \ldots$ & 538 & 848 & $+\mathrm{I}_{5} \mathrm{I}$ & II 8.2 & o. 76 & 4,4 & 3 & 26-inch & 600 & $\mathrm{Br}$. & \\
\hline I898. I I 2 & $o \Sigma$ I $19 \ldots \ldots \ldots \ldots$ & 542 & 229 & +756 & 320.2 & 0.69 & 4,4 & 3 & 26-inch & 600 & $\mathrm{Br}$. & \\
\hline $1903 \cdot 172$ & $\beta 94=61$ Leporis . . & 545 & 54 & -1431 & 182.4 & 2.66 & 4,6 & 3 & 26-inch & 600 & Sk. & $\begin{array}{l}\text { M ags. } 6.0-8.5 \\
\text { white, blue. }\end{array}$ \\
\hline $1903 \cdot 172$ & $\beta \times 6=3$ Monocerotis . . . & 557 & 78 & -1036 & 356.3 & I. 75 & 4,6 & 3 & 26-inch & 600 & Sk. & White, blue. \\
\hline 1900.828 & Skinner $2 \ldots \ldots \ldots \ldots$ & 559 & 936 & $-154 I$ & r69. I & 4. 82 & 4,8 & . & I2-inch & $\cdots$ & B. & Mags. 8.0-9.5. \\
\hline I898. I 12 & $\Sigma 840(\mathrm{~B}$ and $\mathrm{C}) \ldots$ & 60 & ○ 55 & tro 46 & I7I. I & 0.80 & 4,4 & 3 & 26-inch & 600 & $\mathrm{Br}$. & Mags. 9.0-10.0. \\
\hline 1898. I 7 & $o \Sigma 132 \ldots \ldots \ldots \ldots$ & $6 \mathrm{I}$ & I 20 & $+38 \cdot 0$ & 319.9 & I. 76 & 4,4 & 2 & 26-inch & 600 & $\mathrm{Br}$ & Thick. \\
\hline 1903.117 & Hu ro6........ & 64 & 439 & - II 40 & 334.8 & 0.92 & 4,6 & p & 26-inch & $\ldots$ & D. & Mags. 9.2-9.6. \\
\hline
\end{tabular}




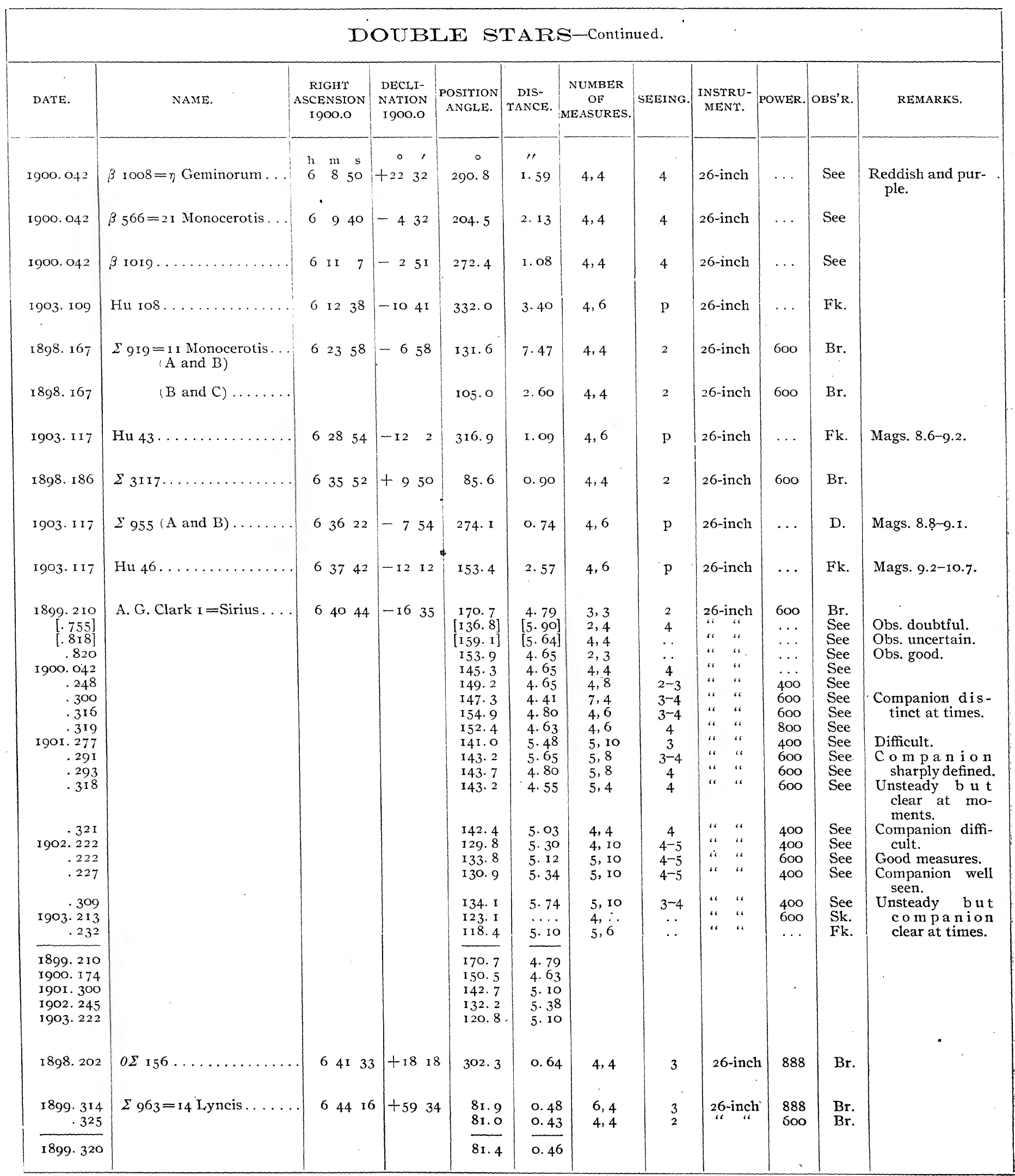


DOUBLE STARS-Continued.

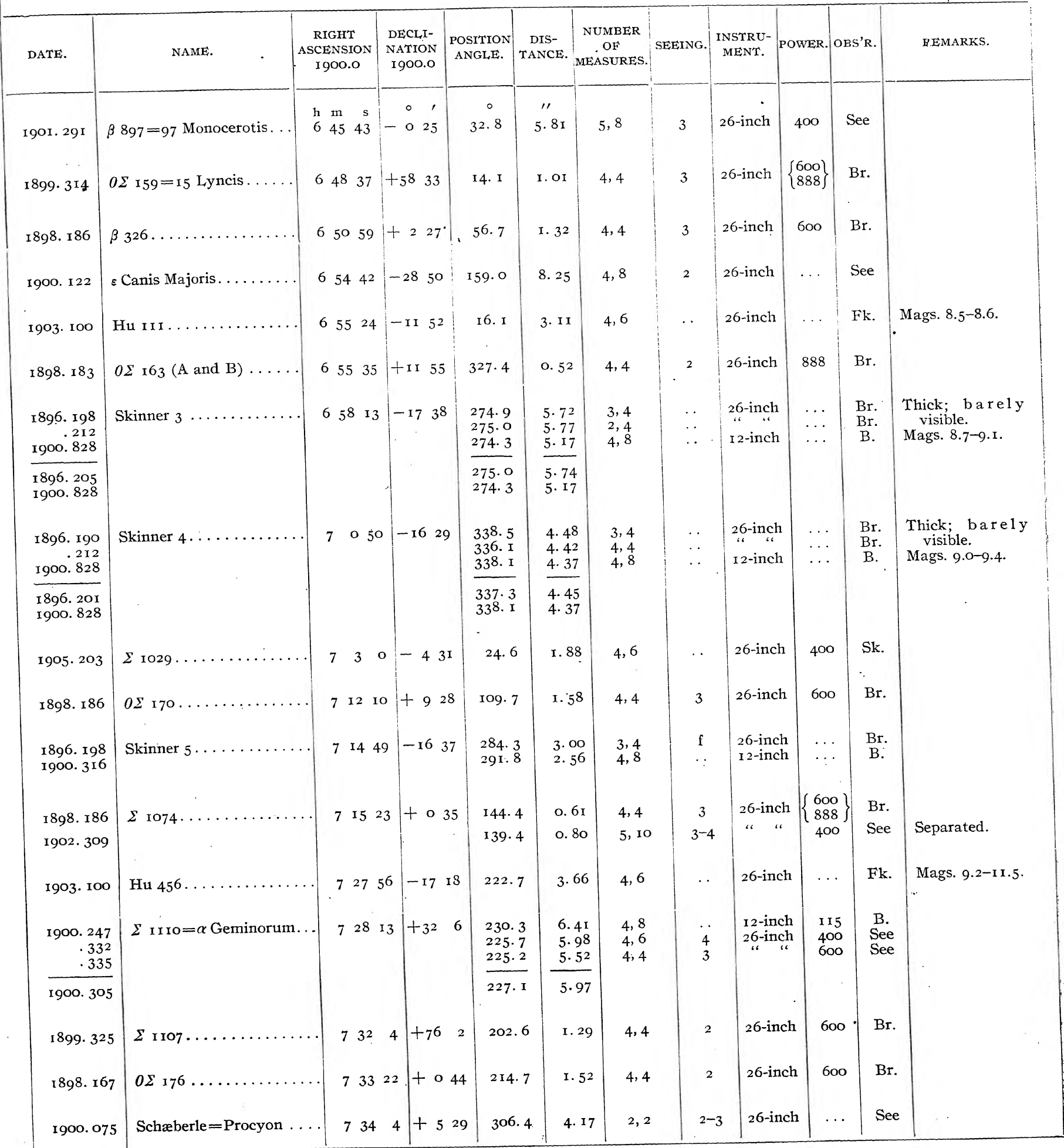




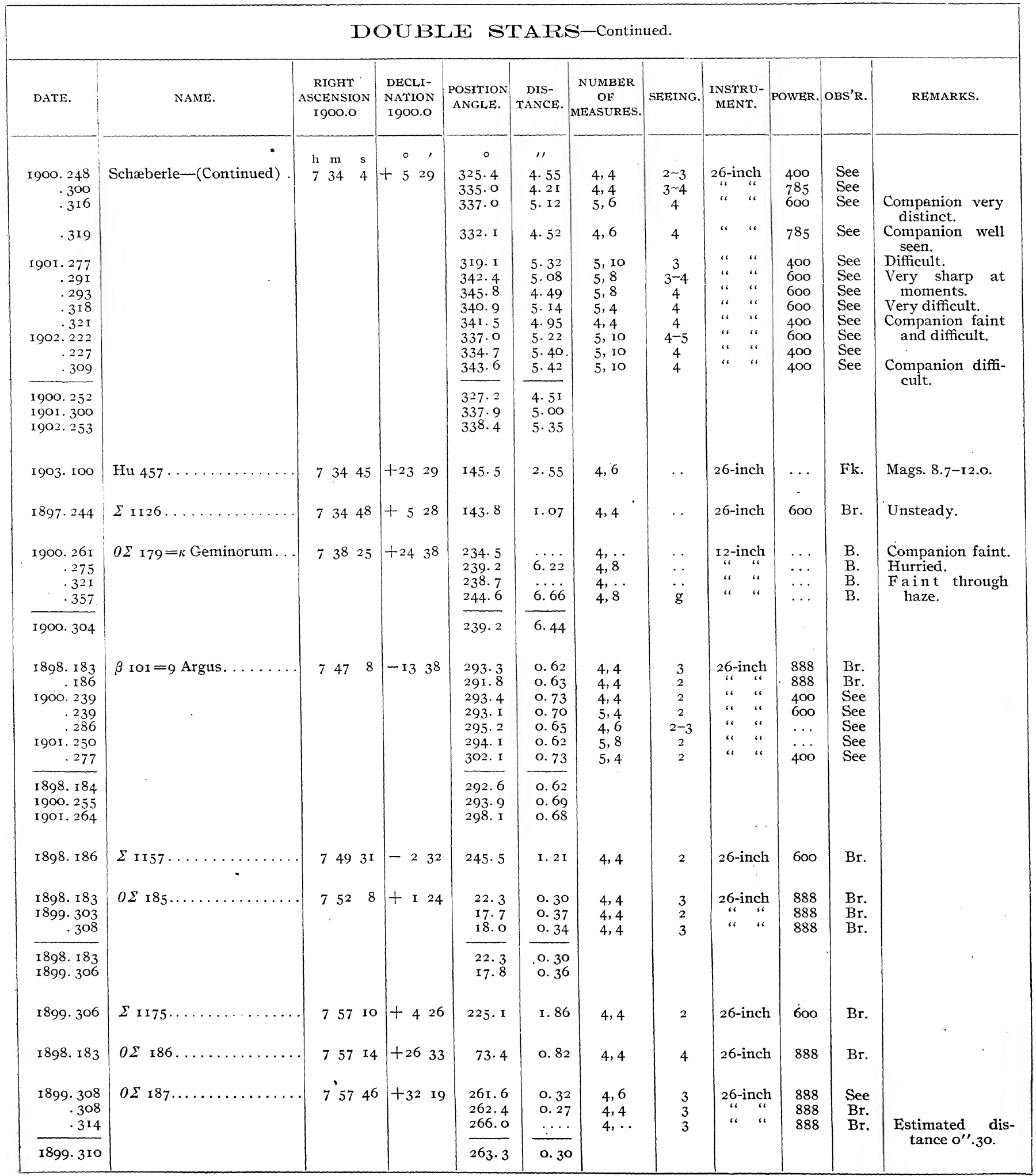




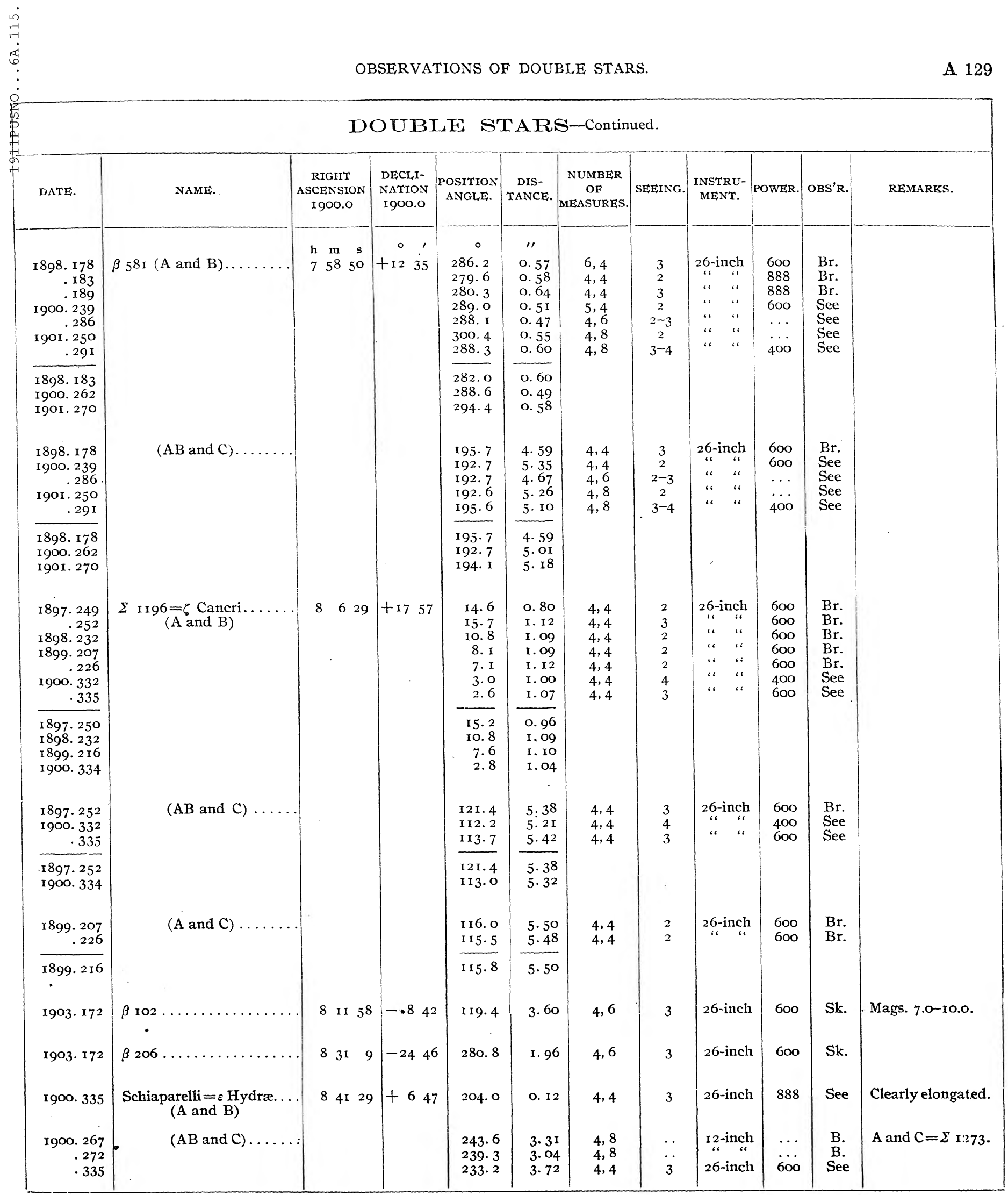

$28167^{\circ}-$ VOL $6-1 \mathrm{I} \longrightarrow 9$ 


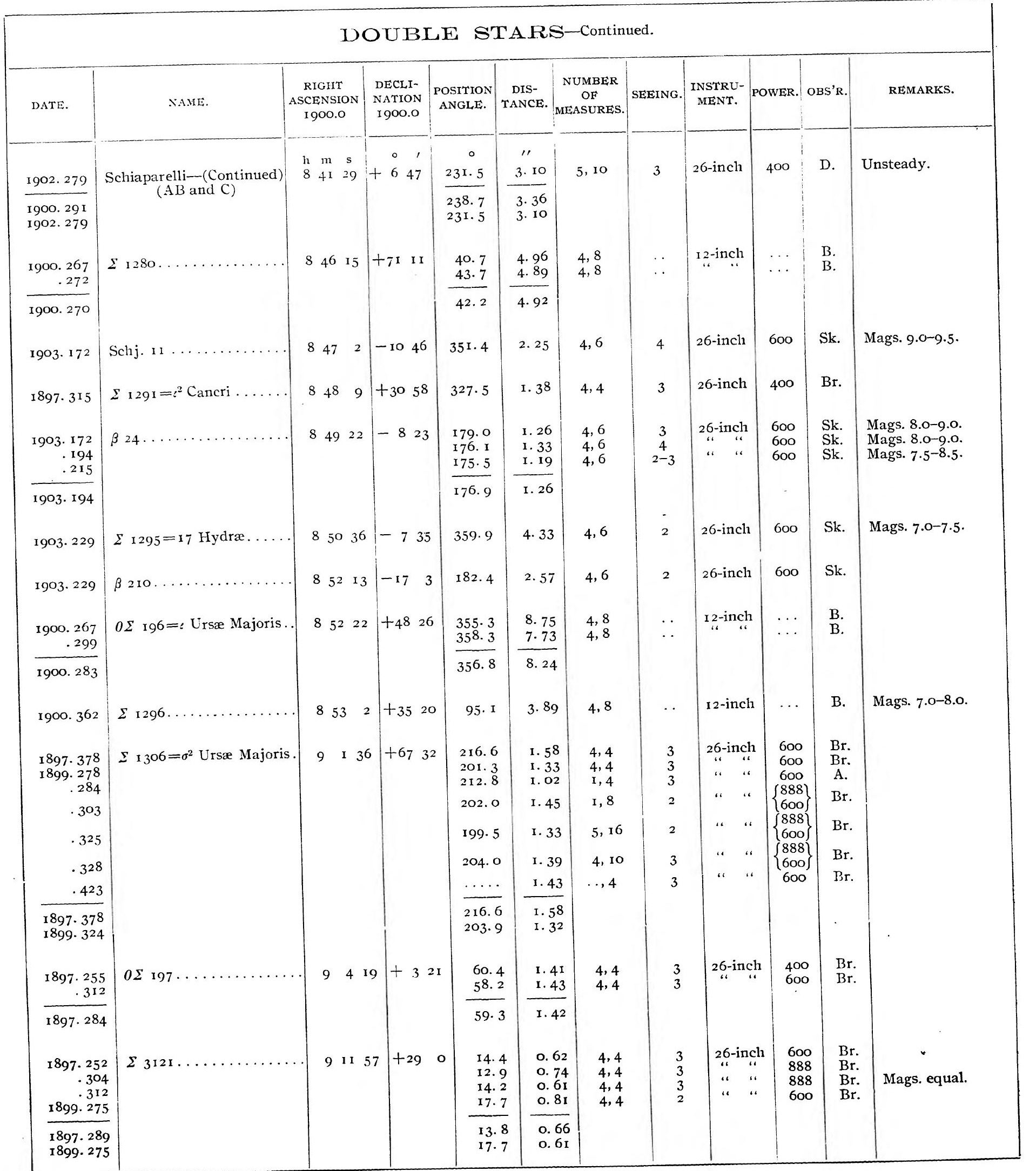


IOOUBIE STARS-Continued.

\begin{tabular}{|c|c|c|c|c|c|c|c|c|c|c|c|c|}
\hline नु & NAME. & $\begin{array}{r}\text { RIGHT } \\
\text { ASCENSIO } \\
\text { I9OO.O }\end{array}$ & & $\begin{array}{c}\text { DECLI- } \\
\text { NATION } \\
\text { I } 900.0\end{array}$ & $\begin{array}{l}\text { POSITION } \\
\text { ANGLE. }\end{array}$ & $\begin{array}{c}\text { DIS- } \\
\text { TANCE. }\end{array}$ & $\begin{array}{c}\text { NUMBER } \\
\text { OF } \\
\text { MEASURES. }\end{array}$ & SEEING. & $\begin{array}{c}\text { INSTRU- } \\
\text { MENT. }\end{array}$ & POWER. & OBS'R. & REMARKS. \\
\hline $\begin{array}{r}1897.244 \\
1900.261 \\
.267\end{array}$ & $\Sigma^{\prime} 1334=38$ Lyncis. . . . . & $\begin{array}{ll}\mathrm{h} & \mathrm{m} \\
9 & \mathrm{I} 2\end{array}$ & $\begin{array}{r}5 \\
37\end{array}$ & $\begin{array}{r}0 \\
+3714\end{array}$ & $\begin{array}{c}235.8 \\
240.5 \\
245.0\end{array}$ & $\begin{array}{l}3.00 \\
2.93 \\
3.25\end{array}$ & $\begin{array}{l}4,4 \\
4,8 \\
4,8\end{array}$ & $\begin{array}{l}\cdots \\
\cdots \\
\cdots\end{array}$ & $\begin{array}{l}\text { 26-inch } \\
12 \text {-inch } \\
\text {. } . .\end{array}$ & $\begin{array}{l}400 \\
\cdots \\
\cdots\end{array}$ & $\begin{array}{l}\text { Br. } \\
\text { B. } \\
\text { B. }\end{array}$ & \\
\hline $\begin{array}{l}\text { I } 897 \cdot 244 \\
\text { I } 900.264\end{array}$ & & & & & $\begin{array}{l}235.8 \\
242.8\end{array}$ & $\begin{array}{l}3.00 \\
3.09\end{array}$ & & & & & & . \\
\hline $\begin{array}{r}1897 \cdot 329 \\
\cdot 373 \\
\cdot 378\end{array}$ & $\Sigma$ I 33 I $(A$ and $B) \ldots \ldots$ & 9 I2 5 & $5^{8}$ & +6146 & $\begin{array}{l}152.7 \\
151.7 \\
153.7\end{array}$ & $\begin{array}{l}0.8 \mathrm{I} \\
0.80 \\
0.84\end{array}$ & $\begin{array}{l}4,4 \\
4,4 \\
4,4\end{array}$ & $\begin{array}{l}2 \\
2 \\
3\end{array}$ & 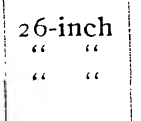 & $\begin{array}{l}400 \\
600 \\
600\end{array}$ & $\begin{array}{l}\mathrm{Br} . \\
\mathrm{Br} . \\
\mathrm{Br}\end{array}$ & \\
\hline $1897 \cdot 360$ & & & & & I 52.7 & 0.82 & & & & & & \\
\hline $\begin{array}{l}\text { I } 897 \cdot 3 \text { I } 5 \\
\text { 1900. } 362\end{array}$ & $\Sigma 1338=157$ Lyncis..... & 9 I4 4 & 44 & $+3^{8} 37$ & $\begin{array}{l}165 \cdot 5 \\
165.0\end{array}$ & $\begin{array}{l}\text { I. } 57 \\
2.31\end{array}$ & $\begin{array}{l}4,4 \\
4,8\end{array}$ & $\begin{array}{l}3 \\
\cdots\end{array}$ & $\begin{array}{l}\text { 26-inch } \\
\text { I 2-inch }\end{array}$ & $\begin{array}{l}400 \\
\cdots\end{array}$ & $\begin{array}{l}\text { Br. } \\
\text { B. }\end{array}$ & \\
\hline $1907 \cdot 311$ & $\beta 337 \ldots \ldots \ldots \ldots \ldots \ldots$ & .9175 & & - I7 28 & 330.3 & $7 \cdot 5^{2}$ & 4,4 & $\mathrm{f}-\mathrm{p}$ & I 2 -inch & 2.35 & $\mathrm{R}$. & Mags. 7.5-10.0. \\
\hline $\begin{array}{r}\text { I897. } 329 \\
.378 \\
\text { I900. } 267 \\
.275 \\
\end{array}$ & $0 \Sigma 200 \ldots \ldots \ldots \ldots$ & 918 & o & $+5^{2} \quad 0$ & $\begin{array}{l}333.9 \\
332.0 \\
325.3 \\
329.7 \\
\end{array}$ & $\begin{array}{l}\text { I. } 47 \\
\text { I. } 53 \\
2.43 \\
\text { I. } 74 \\
\end{array}$ & $\begin{array}{l}4,4 \\
4,4 \\
4,8 \\
4,8\end{array}$ & $\begin{array}{l}2 \\
3 \\
\cdots \\
\cdots\end{array}$ & \begin{tabular}{c|}
26 -inch \\
“" \\
12-inch \\
"
\end{tabular} & $\begin{array}{c}400 \\
600 \\
\cdots \\
\cdots\end{array}$ & $\begin{array}{l}\mathrm{Br} . \\
\mathrm{Br} . \\
\mathrm{B} . \\
\mathrm{B} .\end{array}$ & \\
\hline $\begin{array}{l}\text { I } 897 \cdot 354 \\
1900.271\end{array}$ & & & & & $\begin{array}{l}333 \cdot 0 \\
327 \cdot 5\end{array}$ & $\begin{array}{l}\text { I. } 50 \\
2.08\end{array}$ & & & & & & \\
\hline $1897 \cdot 315$ & $0 \Sigma 201 \ldots \ldots \ldots \ldots$ & 918 & 2 & +2820 & 222.0 & I. 35 & 4,4 & 2 & 26-inch & 600 & $\mathrm{Br}$. & \\
\hline $1907 \cdot 311$ & $\beta 338 \ldots \ldots \ldots \ldots$ & 918 & 12 & $\begin{array}{ll}-15 & 4\end{array}$ & 272.1 & 6.75 & 4,4 & $\mathrm{f}-\mathrm{p}$ & I2-inch & 235 & $\mathrm{R}$. & Mags. 8.5-9.8. \\
\hline $\mathbf{1} 897.255$ & $\Sigma$ I $348=$ I 6 Hydræ..... & 919 & I 3 & +647 & $324 \cdot 3$ & I. 82 & 4,4 & 2 & 26-inch & 400 & $\mathrm{Br}$. & \\
\hline $1897 \cdot 290$ & $\Sigma_{1355 \ldots \ldots \ldots \ldots \ldots}$ & 922 & $\mathbf{I}$ & +640 & 333.8 & 2.67 & 4,4 & 2 & 26-inch & 400 & $\mathrm{Br}$. & \\
\hline $\begin{array}{r}1900.316 \\
1903.292 \\
.319\end{array}$ & Skinner $6 \ldots \ldots \ldots \ldots$ & 922 & 26 & -1650 & $\begin{array}{l}357 \cdot 2 \\
356 \cdot 5 \\
354: 7 \\
\end{array}$ & $\begin{array}{l}6.04 \\
6.45 \\
6.27\end{array}$ & $\begin{array}{l}4,8 \\
4,6 \\
4,6\end{array}$ & $\begin{array}{c}\cdots \\
4-5\end{array}$ & $\begin{array}{l}\text { 12-inch } \\
26 \text {-inch }\end{array}$ & $\begin{array}{l}\cdots \\
400 \\
600\end{array}$ & $\begin{array}{l}\text { B. } \\
\text { Sk. } \\
\text { Sk. }\end{array}$ & $\begin{array}{l}\text { Mags. 9.0-10.o. } \\
\text { Mags. 8.4-9.5. }\end{array}$ \\
\hline $\begin{array}{l}1900.316 \\
1903 \cdot 306\end{array}$ & . & & & & $\begin{array}{l}357 \cdot 2 \\
355 \cdot 6\end{array}$ & $\begin{array}{l}6.04 \\
6.36\end{array}$ & & & & & & \\
\hline $\begin{array}{r}1897.304 \\
.307 \\
1899.207 \\
.226 \\
1900.335 \\
1902.317 \\
.328\end{array}$ & $\Sigma$ I $356=\omega$ Leonis $\ldots \ldots$ & 923 & 6 & +930 & $\begin{array}{l}\text { 106. } \\
\text { I } 12.3 \\
\text { I } 111.6 \\
\text { I } 10.7 \\
\text { I } 12.7 \\
\text { I } 13.4 \\
\text { I } 13.7\end{array}$ & $\begin{array}{l}0.87 \\
0.82 \\
0.85 \\
0.83 \\
0.67 \\
0.92 \\
0.84\end{array}$ & $\begin{array}{l}4,4 \\
4,4 \\
4,4 \\
4,4 \\
4,6 \\
5,10 \\
5,10\end{array}$ & $\begin{array}{c}3 \\
2 \\
2 \\
2 \\
2 \\
2 \\
3-4\end{array}$ & 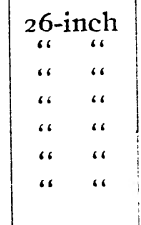 & $\begin{array}{l}600 \\
600 \\
600 \\
600 \\
600 \\
400 \\
600\end{array}$ & $\begin{array}{l}\text { Br. } \\
\text { Br. } \\
\text { Br. } \\
\text { Br. } \\
\text { See } \\
\text { See } \\
\text { See }\end{array}$ & $\begin{array}{l}\text { Clouds. } \\
\text { Unsteady. } \\
\text { Blurred. }\end{array}$ \\
\hline $\begin{array}{l}\text { I } 897 \cdot 306 \\
\text { I } 899 \cdot 2 \text { 1 } 6 \\
1900.335 \\
\text { I } 902.322\end{array}$ & & & & & $\begin{array}{l}109.6 \\
111.2 \\
112.1 \\
113.6\end{array}$ & $\begin{array}{l}\text { o. } 84 \\
\text { o. } 84 \\
\text { o. } 67 \\
\text { o. } 88\end{array}$ & & & 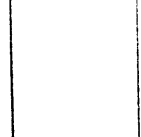 & & & \\
\hline I903. 235 & $\beta 591 \ldots \ldots \ldots \ldots \ldots$ & 924 & 33 & $-24 I$ & 38.9 & o. 99 & 4,6 & 3 & 26-inch & 600 & Sk. & Mags. $8.5-9.5$ \\
\hline $\begin{array}{r}\text { I900. } 267 \\
.275\end{array}$ & $\Sigma$ i374=30 Leo. Minoris. . & 935 & I I & +3924 & $\begin{array}{l}284 \cdot 7 \\
282.6\end{array}$ & $\begin{array}{l}3.25 \\
3.25 \\
\end{array}$ & $\begin{array}{l}4,8 \\
4,8\end{array}$ & $\begin{array}{l}\cdots \\
\cdots\end{array}$ & r 2 -inch & $\begin{array}{l}\cdots \\
\cdots\end{array}$ & $\begin{array}{l}\text { B. } \\
\text { B. }\end{array}$ & \\
\hline 1900. 27 I & & & & & 283.6 & 3.25 & & & & & & \\
\hline
\end{tabular}




\begin{tabular}{|c|c|c|c|c|c|c|c|c|c|c|c|}
\hline \multicolumn{12}{|c|}{ DOUBLE STARS-Continued. } \\
\hline DATE. & NAME. & \begin{tabular}{|} 
RIGHT \\
ASCENSION \\
I9OO.O
\end{tabular} & $\begin{array}{l}\text { DECLI- } \\
\text { NATION } \\
\text { I } 900.0\end{array}$ & $\begin{array}{l}\text { POSITION } \\
\text { ANGLE. }\end{array}$ & $\begin{array}{c}\text { DIS- } \\
\text { TANCE. }\end{array}$ & $\begin{array}{c}\text { NUMBER } \\
\text { OF } \\
\text { MEASURES. }\end{array}$ & SEEING. & $\begin{array}{c}\text { INSTRU- } \\
\text { MENT. }\end{array}$ & POWER. & OBS'R. & REMARKS. \\
\hline $\begin{array}{r}1902.279 \\
.328 \\
\end{array}$ & $\Sigma$ I $377 \ldots \ldots \ldots \ldots \ldots$ & $\begin{array}{ccc}\text { h } & \mathbf{m} & \mathrm{s} \\
9 & 38 & \mathrm{I} 6\end{array}$ & $\begin{array}{r}01 \\
+35\end{array}$ & $\begin{array}{r}\circ \\
138.7 \\
138.9 \\
\end{array}$ & $\begin{array}{l}\prime \prime \\
4.00 \\
3.95\end{array}$ & $\begin{array}{l}5,10 \\
5,10\end{array}$ & $\begin{array}{c}3 \\
2-3\end{array}$ & $\underset{" \text { "inch }}{ }$ & $\begin{array}{l}400 \\
600\end{array}$ & $\begin{array}{l}\text { D. } \\
\text { See }\end{array}$ & \\
\hline $1902 \cdot 304$ & & & & 138.8 & $3 \cdot 98$ & & & & & & \\
\hline $\begin{array}{l}1898.232 \\
1902.328\end{array}$ & $\Sigma x_{3} 85 \ldots \ldots$ & 94429 & +172 & $\begin{array}{l}35 I \cdot I \\
345 \cdot 9\end{array}$ & $\begin{array}{l}\text { I. } 08 \\
\text { I. } 18\end{array}$ & $\begin{array}{l}4,4 \\
5,10\end{array}$ & $\begin{array}{c}2 \\
2-3\end{array}$ & 26-inch & $\begin{array}{l}600 \\
600\end{array}$ & $\begin{array}{l}\text { Br. } \\
\text { See }\end{array}$ & $\begin{array}{l}\text { Seeing bad. } \\
\text { Unsteady. }\end{array}$ \\
\hline $\begin{array}{r}1899.300 \\
.314 \\
.325 \\
\end{array}$ & $O \Sigma 208=\phi$ Urs. Majoris. & $\begin{array}{lll}9 & 45 & 18\end{array}$ & +5432 & $\begin{array}{l}286.2 \\
280.9 \\
283.0 \\
\end{array}$ & $\begin{array}{l}0.40 \\
0.39 \\
0.39 \\
\end{array}$ & $\begin{array}{l}3,4 \\
4,4 \\
4,4\end{array}$ & $\begin{array}{l}3 \\
2 \\
2\end{array}$ & $\begin{array}{c}\text { 26-inch } \\
\text { “" } " ،\end{array}$ & $\begin{array}{l}888 \\
888 \\
888\end{array}$ & $\begin{array}{l}\mathrm{Br} . \\
\mathrm{Br} . \\
\mathrm{Br} .\end{array}$ & . \\
\hline $1899 \cdot 313$ & & & & $283 \cdot 4$ & 0.39 & & & & & & - \\
\hline $\begin{array}{r}1897.290 \\
.323 \\
\end{array}$ & $\Sigma$ I $389 \ldots \ldots \ldots \ldots \ldots$ & $946 \quad 4 \mathrm{I}$ & +2727 & $\begin{array}{l}3112.3 \\
311.2 \\
\end{array}$ & $\begin{array}{l}2.15 \\
2.36 \\
\end{array}$ & $\begin{array}{l}4,4 \\
4: 4\end{array}$ & $\begin{array}{l}2 \\
2\end{array}$ & 26-inch & $\begin{array}{l}400 \\
400\end{array}$ & $\begin{array}{l}\mathrm{Br} . \\
\mathrm{Br} .\end{array}$ & \\
\hline I 897.306 & & & & 311.8 & 2.26 & & & & & & \\
\hline $\begin{array}{r}1897.353 \\
.378 \\
1900.267 \\
.272 \\
\end{array}$ & $\Sigma{ }_{13} 86 \ldots \ldots \ldots \ldots \ldots$ & 94650 & +6922 & $\begin{array}{l}291.5 \\
293 \cdot 1 \\
290.7 \\
296.5 \\
\end{array}$ & $\begin{array}{l}1.94 \\
1.89 \\
2.20 \\
2.06 \\
\end{array}$ & $\begin{array}{l}4,3 \\
4,4 \\
4,8 \\
4,8\end{array}$ & $\begin{array}{l}2 \\
3 \\
\cdots \\
\cdots\end{array}$ & $\begin{array}{l}26 \text {-inch } \\
\text { " " } \\
\text { 1 2-inch } \\
\text { " " }\end{array}$ & $\begin{array}{l}600 \\
600 \\
\cdots \\
\cdots\end{array}$ & $\begin{array}{l}\mathrm{Br} . \\
\mathrm{Br} . \\
\mathrm{B} . \\
\mathrm{B} .\end{array}$ & \\
\hline $\begin{array}{l}\text { I } 897 \cdot 3^{66} \\
\text { 1900. } 270\end{array}$ & & & & $\begin{array}{l}292.3 \\
293.6\end{array}$ & $\begin{array}{l}1.92 \\
2.13\end{array}$ & & & & & & \\
\hline $\begin{array}{r}1897.312 \\
1900.286 \\
.300 \\
.316 \\
1901.250 \\
.277 \\
.291 \\
.318 \\
1902.312 \\
.317 \\
.328 \\
\end{array}$ & $\begin{array}{c}\text { A. Clark } 5=8 \text { Sextantis } \\
(A \text { and } B)\end{array}$ & 94733 & -738 & $\begin{array}{r}\text { IO2. } \\
89.7 \\
87.5 \\
85.4 \\
95.5 \\
95.2 \\
96.3 \\
87.1 \\
93.5 \\
86.8 \\
88.3 \\
\end{array}$ & $\begin{array}{l}0.33 \\
0.42 \\
0.45 \\
0.40 \\
0.50 \\
0.64 \\
0.64 \\
0.46 \\
0.48 \\
0.65 \\
0.55\end{array}$ & $\begin{array}{l}4,4 \\
4,6 \\
4,4 \\
4,4 \\
5,8 \\
5,4 \\
4,8 \\
5,8 \\
5,10 \\
5,10 \\
5,10\end{array}$ & $\begin{array}{c}3 \\
3-4 \\
3-4 \\
4 \\
2 \\
2 \\
3-4 \\
4 \\
2-4 \\
3 \\
3\end{array}$ & 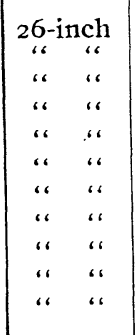 & $\begin{array}{l}888 \\
785 \\
785 \\
\ldots . \\
400 \\
400 \\
600 \\
600 \\
400 \\
600\end{array}$ & $\begin{array}{l}\text { Br. } \\
\text { See } \\
\text { See } \\
\text { See } \\
\text { See } \\
\text { See } \\
\text { See } \\
\text { See } \\
\text { D. } \\
\text { See } \\
\text { See }\end{array}$ & $\begin{array}{l}\text { Clearly separated. } \\
\text { M a g s. 6.5 - 7.0; } \\
\text { both yellow. } \\
\text { Well divided at } \\
\text { moments. } \\
\text { Separated. } \\
\text { Tremulous. }\end{array}$ \\
\hline $\begin{array}{l}\text { I897.31 } 2 \\
1900.301 \\
\text { I901.284 } \\
\text { I9O2.319 }\end{array}$ & & & & $\begin{array}{r}102.2 \\
87.5 \\
93.5 \\
89.5\end{array}$ & $\begin{array}{l}\text { o. } 33 \\
0.42 \\
0.56 \\
0.5^{6}\end{array}$ & & & & & & \\
\hline $\begin{array}{r}1901.277 \\
.291 \\
.318 \\
\end{array}$ & $(\mathrm{AB}$ and $\mathrm{C})$ & & & $\begin{array}{r}325.8 \\
324.4 \\
325.8 \\
\end{array}$ & $\begin{array}{r}35.64 \\
36.25 \\
35.40 \\
\end{array}$ & $\begin{array}{l}5,8 \\
4,8 \\
5,8\end{array}$ & $\begin{array}{c}2 \\
3-4 \\
4\end{array}$ & $\mid$\begin{tabular}{cc|}
$26-$ inch \\
“" & " \\
&
\end{tabular} & $\begin{array}{l}400 \\
400 \\
600\end{array}$ & $\begin{array}{l}\text { See } \\
\text { See } \\
\text { See }\end{array}$ & \\
\hline I901. 295 & & & & $325 \cdot 3$ & $35 \cdot 76$ & & & & & & \\
\hline $1907 \cdot 311$ & $\mathrm{H} 825 \ldots \ldots \ldots$ & 95444 & -1429 & $313 \cdot 4$ & $9 \cdot 96$ & 4,4 & $f-p$ & r2-inch & .160 & $\mathrm{R}$. & Mags. 9.0-9.8. \\
\hline 1899.278 & $\Sigma \times 406 \ldots \ldots \ldots$ & 95952 & +3134 & 229.2 & I. 14 & 4,4 & 3 & 26-inch & 600 & $\mathrm{Br}$. & \\
\hline $\begin{array}{r}1903.194 \\
.235 \\
1905.282 \\
\end{array}$ & $\mathrm{Hd} 125 \ldots \ldots \ldots \ldots$ & Io $\quad$ I 49 & $\mid \begin{array}{ll}-1 & 14\end{array}$ & $\begin{array}{l}173.6 \\
174.4 \\
176.8 \\
\end{array}$ & $\begin{array}{l}2.20 \\
2.27 \\
2.43 \\
\end{array}$ & $\begin{array}{l}4,6 \\
4,6 \\
4,6\end{array}$ & $\underset{3}{4-5}$ & $\begin{array}{l}\text { 26-inch } \\
\text { "، } " ،\end{array}$ & $\begin{array}{l}600 \\
600 \\
400\end{array}$ & $\begin{array}{l}\text { D. } \\
\text { Sk. } \\
\text { Sk. }\end{array}$ & $\begin{array}{l}\text { Mags. } 8.5-8.7 \\
\text { Mags. } 9.0-9.5 \\
\text { Mags. } 9.0-9.1\end{array}$ \\
\hline $\begin{array}{l}1903.214 \\
1905.282\end{array}$ & & & & $\begin{array}{l}174.0 \\
176.8\end{array}$ & $\begin{array}{l}2.24 \\
2.43\end{array}$ & & & & & & \\
\hline
\end{tabular}


DOUBLE STARS-Continued.

\begin{tabular}{|c|c|c|c|c|c|c|c|c|c|c|c|c|}
\hline DATE. & NAME. & $\begin{array}{c}\text { RIGHT } \\
\text { ASCENSIO } \\
\text { I9OO.O }\end{array}$ & & $\begin{array}{c}\text { DECLI- } \\
\text { NATION } \\
\text { I9OO.O }\end{array}$ & $\begin{array}{l}\text { POSITION } \\
\text { ANGLE. }\end{array}$ & $\begin{array}{c}\text { DIS- } \\
\text { TANCE. }\end{array}$ & $\begin{array}{c}\text { NUMBER } \\
\text { OF } \\
\text { MEASURES. }\end{array}$ & SEEING. & $\begin{array}{c}\text { INSTRU- } \\
\text { MENT. }\end{array}$ & POWER. & OBS'R. & REMARKS. \\
\hline $1903 \cdot 344$ & $\sum 1416 \ldots \ldots$ & $\begin{array}{rrr}\text { h } & \text { m } \\
\text { IO } & 7 & 2\end{array}$ & & $\begin{array}{r}01 \\
-1536\end{array}$ & $\begin{array}{c}\circ \\
277 \cdot 3\end{array}$ & $\begin{array}{c}\prime \prime \\
\text { II. } 92\end{array}$ & 4,6 & 3 & 26-inch & 600 & Sk. & Mags. 8.0-10.0. \\
\hline I 899.284 & $0 \Sigma 213 \ldots \ldots \ldots$ & I0 73 & & +2755 & $105 \cdot 5$ & 0.80 & 4,4 & 3 & 26-inch & 600 & $\mathrm{Br}$. & \\
\hline $\begin{array}{r}1897.312 \\
.315 \\
.326 \\
1899.275 \\
1902.312 \\
\end{array}$ & $O \Sigma 215 \ldots \ldots$ & 10104 & 49 & $+18 \quad 14$ & $\begin{array}{l}211.4 \\
211.4 \\
209.4 \\
209.1 \\
207.7 \\
\end{array}$ & $\begin{array}{l}0.89 \\
0.84 \\
0.72 \\
0.87 \\
0.70 \\
\end{array}$ & $\begin{array}{l}4,4 \\
4,4 \\
4,4 \\
4,4 \\
5, \text { Io }\end{array}$ & $\begin{array}{l}3 \\
2 \\
2 \\
2 \\
4\end{array}$ & 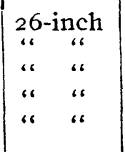 & $\begin{array}{l}600 \\
600 \\
600 \\
600 \\
600\end{array}$ & $\begin{array}{l}\mathrm{Br} . \\
\mathrm{Br} . \\
\mathrm{Br} . \\
\mathrm{Br} . \\
\mathrm{D} .\end{array}$ & $\begin{array}{l}\text { Images very poor. } \\
\text { Woolly. }\end{array}$ \\
\hline $\begin{array}{l}1897 \cdot 318 \\
1899 \cdot 275 \\
1902 \cdot 312\end{array}$ & & & & & $\begin{array}{l}210.7 \\
209.1 \\
207 \cdot 7\end{array}$ & $\begin{array}{l}\text { o. } 82 \\
0.87 \\
0.70\end{array}$ & & & & & & \\
\hline $\mathrm{I} 899.28 \mathrm{I}$ & $0 \Sigma 5_{523}=39$ Leonis. . . & 10 II 4 & & +2336 & 298.5 & $7 \cdot 39$ & 4,4 & 2 & 26-inch & 600 & Br. & \\
\hline $\mathrm{I} 899.28 \mathrm{r}$ & $\Sigma 1423 \ldots \ldots \ldots \ldots \ldots$ & IO I3 4 & 42 & $\begin{array}{ll}-21 & 4\end{array}$ & 58.1 & I. 42 & 4,4 & 2 & 26-inch & 600 & Br. & \\
\hline $\begin{array}{r}\mathrm{I} 897.244 \\
1900.247 \\
.261 \\
.335 \\
\end{array}$ & $\Sigma$ 1 $424=r$ Leonis. . . . . . & 10142 & 28 & $+202 \mathrm{x}$ & $\begin{array}{l}115.2 \\
118.0 \\
116.5 \\
114.0 \\
\end{array}$ & $\begin{array}{r}3.97 \\
4.62 \\
\cdots .7 \\
3 \cdot 78 \\
\end{array}$ & $\begin{array}{l}4,4 \\
4,8 \\
4, \ldots \\
4,4\end{array}$ & $\begin{array}{l}\cdots \\
\ddot{3}\end{array}$ & $\begin{array}{l}\text { 26-inch } \\
\text { 12-inch } \\
\text { 26-inch }\end{array}$ & $\begin{array}{l}400 \\
115 \\
600\end{array}$ & $\begin{array}{l}\text { Br. } \\
\text { B. } \\
\text { B. } \\
\text { See }\end{array}$ & Unsteady. \\
\hline $\begin{array}{l}\text { I } 897.244 \\
\text { I } 900.28 \text { I }\end{array}$ & , & & & & $\begin{array}{l}\text { II } 5.2 \\
\text { I } 6.2\end{array}$ & $\begin{array}{l}3 \cdot 97 \\
4 \cdot 20\end{array}$ & & & & & & \\
\hline $\begin{array}{r}1897 \cdot 304 \\
\cdot 307 \\
\cdot 312 \\
\end{array}$ & $\begin{array}{c}\sum \mathrm{I} 426=\mathrm{I} 45 \mathrm{~L} \text {.eonis. } \ldots \\
(\mathrm{A} \text { and } \mathrm{B})\end{array}$ & IO I5 I & I 8 & +656 & $\begin{array}{l}280.2 \\
280.6 \\
281.6 \\
\end{array}$ & $\begin{array}{l}\text { o. } 87 \\
\text { o. } 92 \\
\text { o. } 80 \\
\end{array}$ & $\begin{array}{l}4,4 \\
4,4 \\
4,4\end{array}$ & $\begin{array}{l}2 \\
2 \\
3\end{array}$ & $\begin{array}{l}26-i n c h \\
" 6 \\
\text { " } 6\end{array}$ & $\begin{array}{l}600 \\
600 \\
600\end{array}$ & $\begin{array}{l}\mathrm{Br} . \\
\mathrm{Br} \\
\mathrm{Br}\end{array}$ & $\begin{array}{l}\text { Windy; telescope } \\
\text { shakes. }\end{array}$ \\
\hline $1897 \cdot 308$ & & & & & 280.8 & o. 86 & & & & & & . \\
\hline $1897 \cdot 312$ & $(A B$ and $C) \ldots$ & & & & 8.4 & $7 \cdot 79$ & 4,4 & 3 & 26-inch & 600 & $\mathrm{Br}$. & \\
\hline $\begin{array}{r}1903 \cdot 344 \\
.350\end{array}$ & Hn $103 \ldots \ldots$ & IO 154 & 45 & -1551 & $\begin{array}{l}338.7 \\
335 \cdot .7 \\
\end{array}$ & $\begin{array}{l}\mathrm{I} .6 \mathrm{I} \\
\mathrm{I} .6 \mathrm{I}\end{array}$ & $\begin{array}{l}4,6 \\
4,6\end{array}$ & $\underset{2-3}{3}$ & 26-inch & $\begin{array}{l}600 \\
600\end{array}$ & $\begin{array}{l}\text { Sk. } \\
\text { Sk. }\end{array}$ & $\begin{array}{l}\text { Mags. } 9 \cdot 0-9 \cdot 5 . \\
\text { Mags. } 9 \cdot 5-10.0 .\end{array}$ \\
\hline $1903 \cdot 347$ & & & & & $337 \cdot 2$ & I. $6 \mathrm{I}$ & & & & & & \\
\hline $1907 \cdot 3$ II & $\mathrm{Hn} 104 \ldots \ldots \ldots \ldots \ldots$ & 1015 & 47 & -1613 & I4. 8 & $3 \cdot 43$ & 4,4 & $f-p$ & 12-inch & 235 & $\mathrm{R}$. & Mags. $9 \cdot 5-9 \cdot 5$ \\
\hline $\begin{array}{r}1903.194 \\
.333 \\
.344 \\
1905.282 \\
\end{array}$ & $\beta_{25}=$ Schj. $16 \ldots \ldots \ldots$ & 10 16 & 46 & $-9 \times 6$ & $\begin{array}{l}179.6 \\
175.5 \\
174.8 \\
175.8 \\
\end{array}$ & $\begin{array}{l}2.44 \\
1.87 \\
2.00 \\
1.91\end{array}$ & $\begin{array}{l}4,6 \\
4,6 \\
4,6 \\
4,6\end{array}$ & $\begin{array}{c}4 \\
3 \\
3 \\
2-3\end{array}$ & 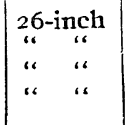 & $\begin{array}{l}600 \\
600 \\
600 \\
400\end{array}$ & $\begin{array}{l}\text { Sk. } \\
\text { Sk. } \\
\text { Sk. } \\
\text { Sk. }\end{array}$ & $\begin{array}{l}\text { Mags. 8.5-r r.o. } \\
\text { Mags. 8.0-8.5. } \\
\text { Mags. 8.5-9.0. } \\
\text { Mags. 8.0-8.5. }\end{array}$ \\
\hline $\begin{array}{l}1903.290 \\
1905.282\end{array}$ & & & & & $\begin{array}{l}176.6 \\
175.8\end{array}$ & $\begin{array}{l}2.10 \\
1.91\end{array}$ & & & & & & \\
\hline $\begin{array}{r}1903.229 \\
.344 \\
.350 \\
\end{array}$ & $\beta 219 \ldots \ldots \ldots \ldots \ldots$ & Io 16 & 52 & $-22 \quad 2$ & $\begin{array}{l}\text { I } 88.5 \\
\text { I } 91.4 \\
193.3 \\
\end{array}$ & $\begin{array}{l}2.43 \\
1.99 \\
2.25 \\
\end{array}$ & $\begin{array}{l}4,6 \\
4,6 \\
4,6\end{array}$ & $\begin{array}{c}2 \\
3 \\
2-3\end{array}$ & $\begin{array}{cc}26-i n c h \\
، & 4 \\
، & ،\end{array}$ & $\begin{array}{l}600 \\
600 \\
600\end{array}$ & $\begin{array}{l}\text { Sk. } \\
\text { Sk. } \\
\text { Sk. }\end{array}$ & $\begin{array}{l}\text { Mags. } 7.0-9.5 \\
\text { Mags. } 8.0-10.0 \\
\text { Mags. } 6.0-9.5\end{array}$ \\
\hline $1903 \cdot 308$ & & & & & I91. I & 2.22 & & & & & & \\
\hline
\end{tabular}









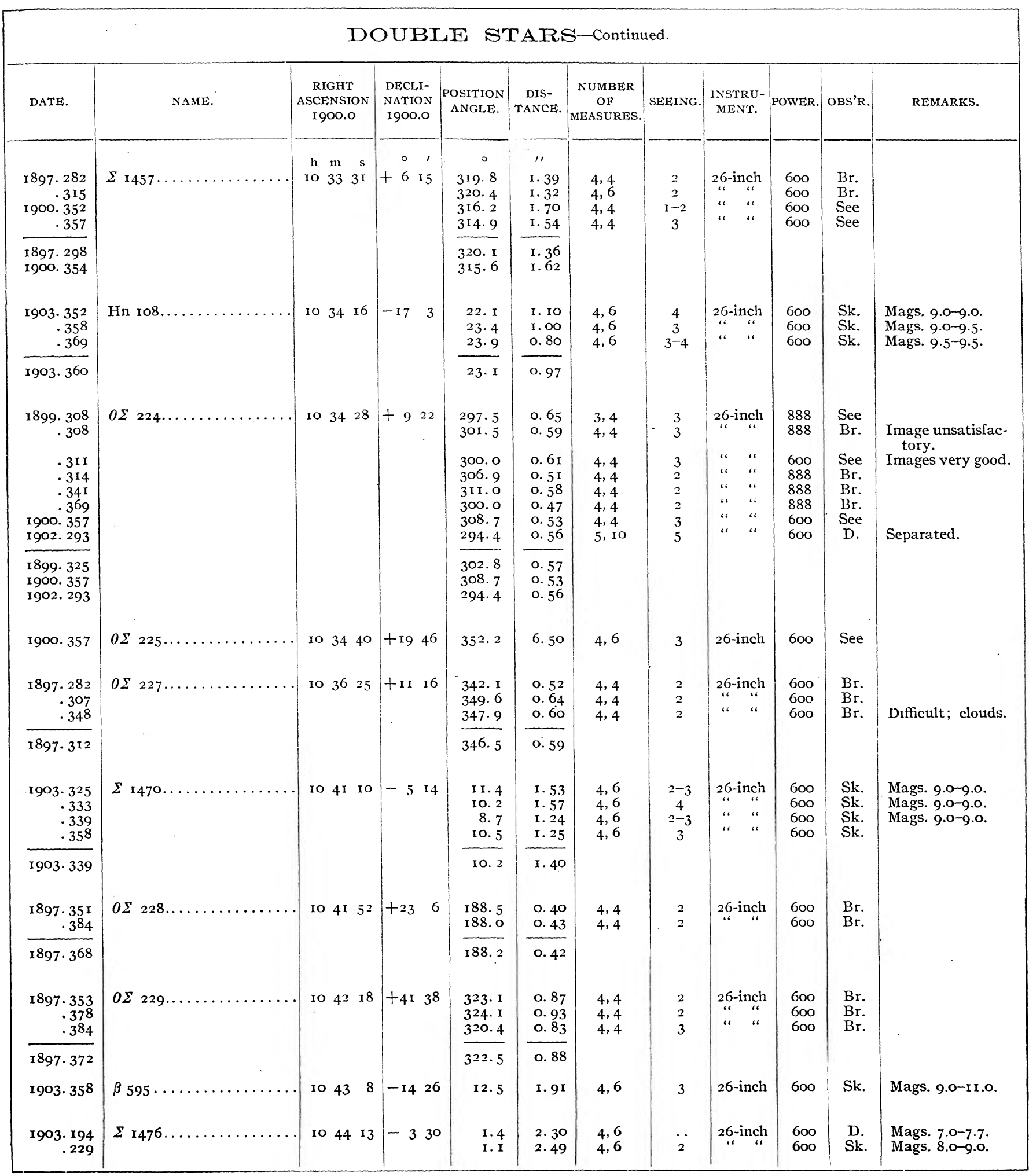




\begin{tabular}{|c|c|c|c|c|c|c|c|c|c|c|c|}
\hline \multicolumn{12}{|c|}{ DOUBLE STARS-Continued. } \\
\hline DATE. & NAME. & $\begin{array}{c}\text { RIGHT } \\
\text { ASCENSION } \\
1900.0\end{array}$ & \begin{tabular}{|} 
DECLI- \\
NATION \\
I9O0.0
\end{tabular} & $\begin{array}{l}\text { POSITION } \\
\text { ANGLE. }\end{array}$ & \begin{tabular}{|c|} 
DIS- \\
TANCE.
\end{tabular} & $\begin{array}{c}\text { NUMBER } \\
\text { OF } \\
\text { MEASURES. }\end{array}$ & SEEING. & $\begin{array}{c}\text { INSTRU- } \\
\text { MENT. }\end{array}$ & POWER. & OBS'R. & REMARKS. \\
\hline $\begin{array}{r}1903.325 \\
.330 \\
.341 \\
\end{array}$ & $\Sigma 1476$ (Continued) $\ldots$ & $\begin{array}{rrr}\mathrm{h} & \mathrm{m} & \mathrm{s} \\
\text { 10 } & 44 & \mathrm{1} 3\end{array}$ & $\left|\begin{array}{rr}0 & 1 \\
-3 & 30\end{array}\right|$ & $\begin{array}{l}\circ \\
\text { 3. } 4 \\
\text { I. } 6 \\
4.2 \\
\end{array}$ & $\begin{array}{l}1 \prime \\
1.93 \\
1.89 \\
1.97 \\
\end{array}$ & $\begin{array}{l}4,6 \\
4,6 \\
4,6\end{array}$ & $\begin{array}{l}2-3 \\
2-3 \\
2-3\end{array}$ & $\begin{array}{c}26-\text { inch } \\
\text { “" } \\
\text { “" }\end{array}$ & $\begin{array}{l}600 \\
600 \\
600\end{array}$ & $\begin{array}{l}\text { Sk. } \\
\text { Sk. } \\
\text { Sk. }\end{array}$ & $\begin{array}{l}\text { Mags. 7.5-7.5. } \\
\text { Mags. 8 0-9.0. } \\
\text { Mags. 7.0-8.o. }\end{array}$ \\
\hline 1903.284 & & & & 2.3 & 2. 12 & & & & & & \\
\hline $\begin{array}{r}1903.333 \\
.339 \\
.358 \\
\end{array}$ & $\beta$ III $\ldots$ & 10 46 10 & -834 & $\begin{array}{l}6.9 \\
6.5 \\
5.4 \\
\end{array}$ & $\begin{array}{l}3.29 \\
3.62 \\
3.49 \\
\end{array}$ & $\begin{array}{l}4,6 \\
4,6 \\
4,6\end{array}$ & $\begin{array}{c}4 \\
2-3 \\
3\end{array}$ & $\begin{array}{c}26-\text {-inch } \\
\text { ". } \\
\text { ". }\end{array}$ & $\begin{array}{l}600 \\
600 \\
600\end{array}$ & $\begin{array}{l}\text { Sk. } \\
\text { Sk. } \\
\text { Sk. }\end{array}$ & $\begin{array}{l}\text { Mags. 10.0-10.o. } \\
\text { Mags. 10.0-10.0. } \\
\text { Mags. 9.5-9.5. }\end{array}$ \\
\hline $1903 \cdot 343$ & & & & 6.3 & $3 \cdot 47$ & & & & & & \\
\hline 1900.357 & $O \Sigma 230 \ldots \ldots \ldots$ & 104910 & $+21 \quad 18$ & $15 \cdot 9$ & 8.54 & 4,4 & 3 & 26-inch & 600 & See & \\
\hline I900. 357 & $\Sigma_{1487}=54$ Leonis $\ldots \ldots$ & 105012 & $+25 \quad 17$ & 104.8 & $6.6 \mathrm{I}$ & 4,4 & 3 & 26-inch & 600 & See & \\
\hline $\begin{array}{r}1897 \cdot 323 \\
.326 \\
1900.357 \\
.371\end{array}$ & $\Sigma$ 1500.. & Io 5456 & $-25^{6}$ & $\begin{array}{l}315.6 \\
312.8 \\
309.6 \\
306.9\end{array}$ & $\begin{array}{l}\text { I. } 22 \\
\text { I. } 57 \\
\text { I. } 75 \\
\text { I. } 80\end{array}$ & $\begin{array}{l}4,4 \\
4,4 \\
4,4 \\
4,4\end{array}$ & $\begin{array}{l}2 \\
2 \\
2 \\
2\end{array}$ & $\begin{array}{cc}26-\text {-inch } \\
\text { “. } \\
\ldots & \text { ". } \\
\text { “. } & \end{array}$ & $\begin{array}{l}600 \\
600 \\
600 \\
600\end{array}$ & $\begin{array}{l}\text { Br. } \\
\text { Br. } \\
\text { See } \\
\text { See }\end{array}$ & $\begin{array}{l}\text { Hazy and very } \\
\text { blurred. }\end{array}$ \\
\hline $\begin{array}{r}\text { 1903. I94 } \\
.273 \\
.325 \\
.330 \\
.347 \\
807\end{array}$ & & & & $\begin{array}{l}310.5 \\
309.8 \\
312.1 \\
310.0 \\
309.5 \\
\end{array}$ & $\begin{array}{l}\text { I. } 64 \\
\text { I. } 69 \\
\text { I. } 95 \\
\text { I. } 67 \\
\text { I. } 69 \\
\end{array}$ & $\begin{array}{l}4,6 \\
4,6 \\
4,6 \\
4,6 \\
4,6\end{array}$ & $\begin{array}{c}4 \\
3 \\
2-3 \\
3 \\
2-3\end{array}$ & 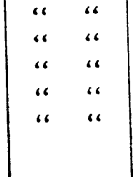 & $\begin{array}{l}600 \\
600 \\
600 \\
600 \\
600\end{array}$ & $\begin{array}{l}\text { Sk. } \\
\text { Sk. } \\
\text { Sk. } \\
\text { Sk. } \\
\text { Sk. }\end{array}$ & $\begin{array}{l}\text { Mags. 7.5-8.5. } \\
\text { Mags. 8 5-9.0. } \\
\text { Mags. 7.0-7.o. } \\
\text { Mags. 7.5-8.o. } \\
\text { Mags. 8.0-8.o. }\end{array}$ \\
\hline $\begin{array}{l}1897.324 \\
1900.364 \\
1903.294\end{array}$ & & & & $\begin{array}{l}314.2 \\
308.2 \\
310.4\end{array}$ & $\begin{array}{l}1.40 \\
\text { I. } 78 \\
\text { I. } 73\end{array}$ & & & & & & \\
\hline $\begin{array}{r}\text { I903. } 339 \\
.341 \\
.344 \\
.35^{2}\end{array}$ & 병. $77 \ldots \ldots \ldots$ & $\begin{array}{lll}10 & 57 & 8\end{array}$ & $\begin{array}{ll}-15 & 9\end{array}$ & $\begin{array}{l}16.1 \\
17.8 \\
16.7 \\
18.3 \\
\end{array}$ & $\begin{array}{l}2.77 \\
2.88 \\
2.85 \\
3.03 \\
\end{array}$ & $\begin{array}{l}4,6 \\
4,6 \\
4,6 \\
4,6\end{array}$ & $\begin{array}{c}2-3 \\
2-3 \\
3 \\
4\end{array}$ & $\begin{array}{c}26-\text { inch } \\
\text { " } \\
.\end{array}$ & $\begin{array}{l}600 \\
600 \\
600 \\
600\end{array}$ & $\begin{array}{l}\text { Sk. } \\
\text { Sk. } \\
\text { Sk. } \\
\text { Sk. }\end{array}$ & $\begin{array}{l}\text { Mags. } 8.5-8.5 . \\
\text { Mags. 9.0-9.0. } \\
\text { Mags. 9.0-9.5. } \\
\text { Mags. 8.5-8.5. }\end{array}$ \\
\hline I903. 344 & & & & 17.2 & 2.88 & & & & & & \\
\hline $\begin{array}{r}1903.352 \\
.358 \\
.363 \\
.369 \\
\end{array}$ & Howe $25 \ldots \ldots \ldots \ldots$ & IO $58 \quad 27$ & -2659 & $\begin{array}{l}333.3 \\
328.3 \\
332.3 \\
331.4 \\
\end{array}$ & $\begin{array}{l}2.34 \\
2.67 \\
2.50 \\
2.26 \\
\end{array}$ & $\begin{array}{l}4,6 \\
4,6 \\
4,6 \\
4,6\end{array}$ & $\begin{array}{c}4 \\
3 \\
3 \\
3-4\end{array}$ & $\begin{array}{c}26-i n c h \\
\text { “" } \\
\text { “" } \\
\text { “ }\end{array}$ & $\begin{array}{l}600 \\
600 \\
600 \\
600\end{array}$ & $\begin{array}{l}\text { Sk. } \\
\text { Sk. } \\
\text { Sk. } \\
\text { Sk. }\end{array}$ & $\begin{array}{l}\text { Mags. 8.5-9.5. } \\
\text { Mags. 8.0-10.0. } \\
\text { Mags. 8.0-10.5. } \\
\text { Mags. 8.0-10.0. }\end{array}$ \\
\hline 1903.360 & & & & 33 I. 3 & 2. 44 & & & & & & \\
\hline $\begin{array}{r}1897.323 \\
.326 \\
1900.357 \\
.376 \\
\end{array}$ & $\Sigma \Sigma_{504} \ldots \ldots$ & 10 $595^{6}$ & $+4 \mathrm{II}$ & $\begin{array}{l}109.7 \\
108.2 \\
104.6 \\
108.9 \\
\end{array}$ & $\begin{array}{l}\text { I. O1 } \\
\text { 1. } 10 \\
1.52 \\
1.07 \\
\end{array}$ & $\begin{array}{l}4,4 \\
4,4 \\
4,4 \\
4,4\end{array}$ & $\begin{array}{c}2 \\
2 \\
1-2 \\
4\end{array}$ & $\begin{array}{cc}26-i n c h \\
، & 4 \\
“ & 4 \\
“ & ،\end{array}$ & $\begin{array}{l}600 \\
600 \\
600 \\
600\end{array}$ & $\begin{array}{l}\mathrm{Br} \\
\mathrm{Br} \\
\text { See } \\
\text { See }\end{array}$ & $\begin{array}{l}\text { Blurred; too bad } \\
\text { to continue. }\end{array}$ \\
\hline $\begin{array}{l}1897.324 \\
1900.366\end{array}$ & & & & $\begin{array}{l}\text { I09. } \\
\text { I06. } 8\end{array}$ & $\begin{array}{l}\text { I. } 06 \\
\text { I. } 30\end{array}$ & & & & & & \\
\hline $\begin{array}{r}1900.286 \\
.300 \\
1901.250\end{array}$ & $\beta 599=65$ Leonis..... & II $\quad$ I 48 & +230 & $\begin{array}{l}82.8 \\
82.7 \\
96.9\end{array}$ & $\begin{array}{l}1.92 \\
1.09 \\
\text { I. } 47\end{array}$ & $\begin{array}{r}4,6 \\
4,4 \\
4,8\end{array}$ & $\begin{array}{c}2 \\
3-4 \\
2\end{array}$ & $\begin{array}{cc}26-\text { inch } \\
\because & 4 \\
& \end{array}$ & $\begin{array}{l}600 \\
\cdots\end{array}$ & $\begin{array}{l}\text { See } \\
\text { See } \\
\text { See }\end{array}$ & $\begin{array}{l}\text { Hazy; very diff- } \\
\text { cult. }\end{array}$ \\
\hline $.32 \mathrm{I}$ & & & & 88.5 & 2.07 & 4,4 & 4 & “ “ & 600 & See & Quite plain \\
\hline $\begin{array}{l}1900.293 \\
1901.286\end{array}$ & & & & $\begin{array}{l}82.8 \\
92.7\end{array}$ & $\begin{array}{l}1.50 \\
1.77\end{array}$ & & & & & & \\
\hline
\end{tabular}




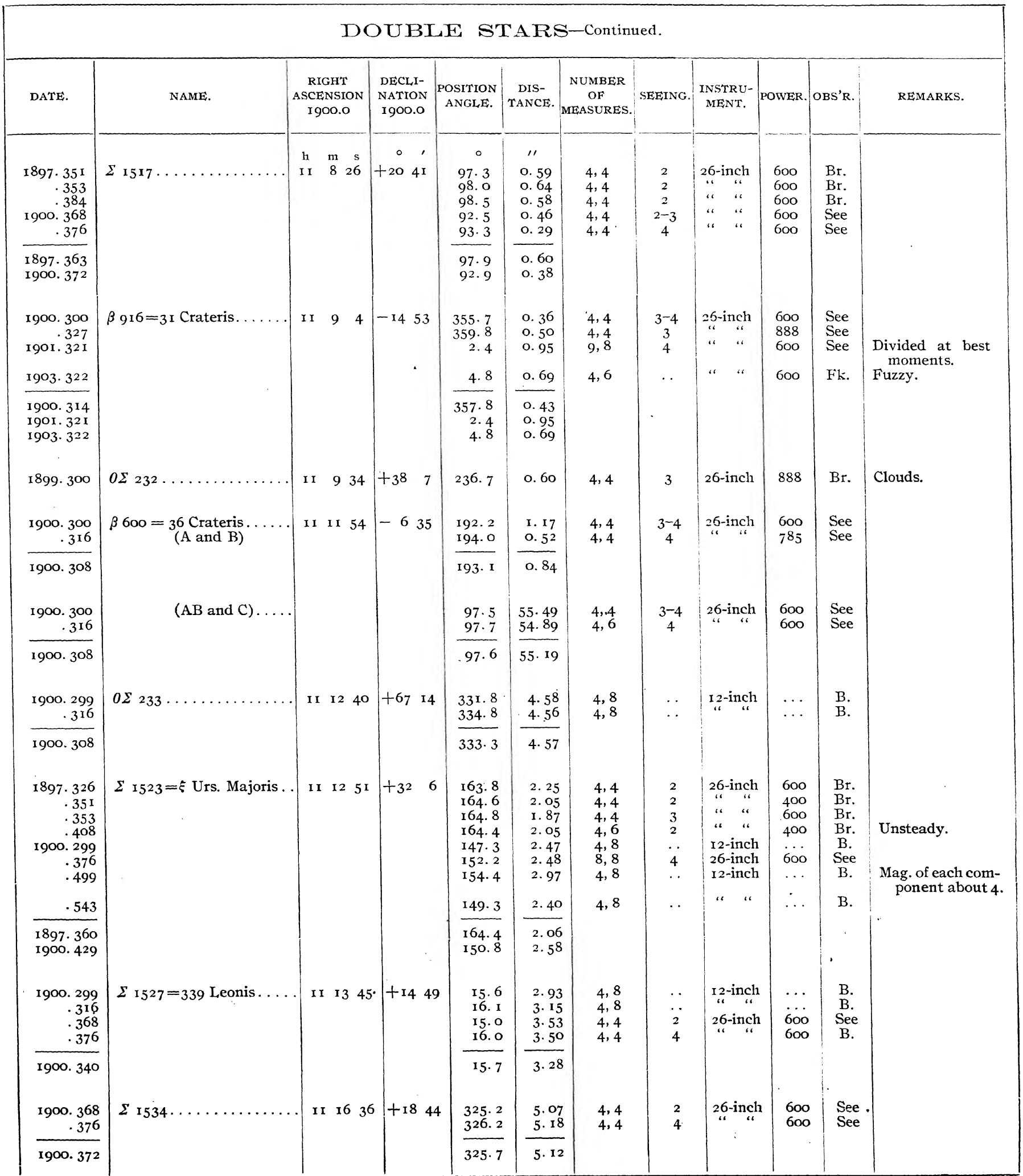




\begin{tabular}{|c|c|c|c|c|c|c|c|c|c|c|c|}
\hline \multicolumn{12}{|c|}{ DOUBLE STARS-Continued. } \\
\hline DATE. & NAME. & $\begin{array}{c}\text { RIGHT } \\
\text { ASCENSION } \\
1900.0\end{array}$ & \begin{tabular}{|c|} 
DECLI- \\
NATION \\
1900.0
\end{tabular} & $\begin{array}{l}\text { POSITION } \\
\text { ANGLE. }\end{array}$ & $\begin{array}{c}\text { DIS- } \\
\text { TANCE. }\end{array}$ & $\begin{array}{c}\text { NUMBER } \\
\text { OF } \\
\text { MEASURES. }\end{array}$ & SEEING. & $\begin{array}{c}\text { INSTRU- } \\
\text { MENT. }\end{array}$ & POWER. & OBS'R. & REMARKS. \\
\hline $\begin{array}{r}\text { I900. } 299 \\
.316 \\
.368 \\
.376 \\
\end{array}$ & $\Sigma$ 1536=: Leonis & $\begin{array}{rrr}\mathrm{h} & \mathrm{m} & \mathrm{s} \\
\mathrm{II} & \mathrm{I} 8 & 43\end{array}$ & $\begin{array}{r}0 \\
+\mathrm{II} \quad 5\end{array}$ & $\begin{array}{c}\circ \\
50.5 \\
51.2 \\
57.3 \\
52.6\end{array}$ & $\begin{array}{c}\prime \prime \\
2.70 \\
2.40 \\
2.78 \\
2.73\end{array}$ & $\begin{array}{l}4,8 \\
4,8 \\
4,4 \\
4,4\end{array}$ & $\begin{array}{l}\cdots \\
2 \\
4\end{array}$ & $\begin{array}{l}\text { 12-inch } \\
\text { 26-inch } \\
\text { “" }\end{array}$ & $\begin{array}{l}\cdots \\
600 \\
600\end{array}$ & $\begin{array}{l}\text { B. } \\
\text { B. } \\
\text { See } \\
\text { See }\end{array}$ & \\
\hline 1900.340 & & & & $52 \cdot 9$ & 2.65 & & & & & & \\
\hline $\begin{array}{r}1900.316 \\
.324 \\
1903.229\end{array}$ & $\beta 26 \ldots \ldots \ldots \ldots$ & II 1843 & -952 & $\begin{array}{l}65.2 \\
66.8 \\
73.4\end{array}$ & $\begin{array}{l}2.82 \\
2.91 \\
3.22\end{array}$ & $\begin{array}{l}4,4 \\
4,4 \\
4,6\end{array}$ & $\begin{array}{c}4 \\
2-3 \\
2\end{array}$ & $\begin{array}{c}26-\text { inch } \\
\text { " } \\
\text { " }\end{array}$ & $\begin{array}{l}600 \\
400 \\
600\end{array}$ & $\begin{array}{l}\text { See } \\
\text { See } \\
\text { Sk. }\end{array}$ & $\begin{array}{l}\text { Mags. } 7.5-9.5 \\
\text { white, blue. }\end{array}$ \\
\hline .292 & & & & $74 \cdot 2$ & 3. ro & 4,6 & $3-4$ & “ " & 600 & Sk. & $\begin{array}{l}\text { Mags. 8.o-r I.o; } \\
\text { white, blue. }\end{array}$ \\
\hline .295 & & & & 67.6 & $2 \cdot 96$ & 4,6 & 4 & “" " & 600 & Sk. & $\begin{array}{l}\text { Mags. 8.o-ro.o; } \\
\text { white, blue. }\end{array}$ \\
\hline .325 & & & & 67.6 & 2.98 & 4,6 & 3 & “ “ & 600 & Sk. & Mags. 8.o-10.0. \\
\hline $\begin{array}{l}1900.320 \\
1903 \cdot 285\end{array}$ & & & & $\begin{array}{l}66.0 \\
70.7\end{array}$ & $\begin{array}{l}2.86 \\
3.06\end{array}$ & & & & & & \\
\hline $\begin{array}{r}1903.344 \\
.347 \\
.350 \\
.3^{8} 5 \\
\end{array}$ & $\mathrm{H} 840=r$ Crateris...$\cdots$ & II 1953 & -178 & $\begin{array}{l}98.4 \\
96.5 \\
98.0 \\
95.6 \\
\end{array}$ & \begin{tabular}{l|}
5.21 \\
5.03 \\
4.99 \\
$5 \cdot 30$ \\
\end{tabular} & $\begin{array}{l}4,6 \\
4,6 \\
4,6 \\
4,6\end{array}$ & $\begin{array}{l}3 \\
2-3 \\
2-3 \\
2-3\end{array}$ & 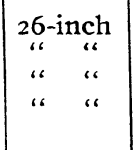 & $\begin{array}{l}600 \\
600 \\
600 \\
600\end{array}$ & $\begin{array}{l}\text { Sk. } \\
\text { Sk. } \\
\text { Sk. } \\
\text { Sk. }\end{array}$ & Mags. 4.0-10.0. \\
\hline $1903 \cdot 35^{6}$ & & & & $97 \cdot \mathrm{I}$ & $5 \cdot 13$ & & & & & & \\
\hline $\begin{array}{r}1900.299 \\
.316 \\
.543 \\
\end{array}$ & $\Sigma_{1543}=57$ Lrs. Majoris. & II $234 \mathrm{I}$ & +3953 & $\begin{array}{l}359 \cdot 5 \\
356.1 \\
358.1 \\
\end{array}$ & $\begin{array}{l}5 \cdot 05 \\
5 \cdot 70 \\
5 \cdot 95 \\
\end{array}$ & $\begin{array}{l}4,8 \\
4,8 \\
4,8\end{array}$ & $\begin{array}{l}\cdots \\
\cdots\end{array}$ & \begin{tabular}{|c|} 
12-inch \\
“" \\
“"
\end{tabular} & $\begin{array}{l}\cdots \\
\cdots \\
\cdots\end{array}$ & $\begin{array}{l}\text { B. } \\
\text { B. } \\
\text { B. }\end{array}$ & \\
\hline 1900.386 & & & 1 & $357 \cdot 9$ & $5 \cdot 57$ & & & & & & \\
\hline \begin{tabular}{r|}
1903.350 \\
.352 \\
.360 \\
.385 \\
\end{tabular} & Jacob $6 \ldots$ & I I 2440 & -2355 & $\begin{array}{l}81.6 \\
80.5 \\
81.9 \\
80.3 \\
\end{array}$ & $\begin{array}{l}8.35 \\
8.22 \\
8.44 \\
8.34\end{array}$ & $\begin{array}{l}4,6 \\
4,6 \\
4,6 \\
4,6\end{array}$ & $\begin{array}{l}2-3 \\
4 \\
2-3 \\
2-3\end{array}$ & $\begin{array}{cc}26-\text { inch } \\
\text { " } \\
، \\
، & 4\end{array}$ & $\begin{array}{l}600 \\
600 \\
600 \\
600\end{array}$ & $\begin{array}{l}\text { Sk. } \\
\text { Sk. } \\
\text { Sk. } \\
\text { Sk. }\end{array}$ & $\begin{array}{l}\text { Mags. 6.0-10.0. } \\
\text { Mags. 6.5-9.5. } \\
\text { Mags. 5.0-9.0. }\end{array}$ \\
\hline $1903 \cdot 362$ & & & & 8 r. 1 & 8. 34 & & & & & & \\
\hline $\begin{array}{r}1900.316 \\
.324 \\
\end{array}$ & $\beta 340 \ldots \ldots \ldots \ldots \ldots$ & II $245^{1}$ & +346 & $\begin{array}{r}6.9 \\
5.2 \\
\end{array}$ & $\begin{array}{l}4.59 \\
3.86 \\
\end{array}$ & $\begin{array}{l}4,4 \\
4,4\end{array}$ & $\begin{array}{c}4 \\
2-3\end{array}$ & 26-inch & $\begin{array}{l}600 \\
400\end{array}$ & $\begin{array}{l}\text { See } \\
\text { See }\end{array}$ & \\
\hline 1900.320 & & & & 6.0 & 4.22 & & & & & & \\
\hline $\begin{array}{r}1897.384 \\
1899.308 \\
.325 \\
.328 \\
\end{array}$ & $0 \Sigma 234 \cdots \cdots \cdots \cdots \cdots \cdots$ & $\begin{array}{lll}11 & 25 & 25\end{array}$ & +4150 & $\begin{array}{l}129.4 \\
137.0 \\
137.1 \\
137.3 \\
\end{array}$ & $\begin{array}{l}0.31 \\
0.26 \\
0.42 \\
0.36\end{array}$ & $\begin{array}{l}4,4 \\
5,4 \\
4,6 \\
7,4\end{array}$ & $\begin{array}{l}3 \\
3 \\
2 \\
4\end{array}$ & 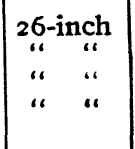 & $\begin{array}{l}888 \\
888 \\
888 \\
888\end{array}$ & $\begin{array}{l}\mathrm{Br} . \\
\mathrm{Br} . \\
\mathrm{Br} . \\
\mathrm{Br} .\end{array}$ & \\
\hline $\begin{array}{l}1897 \cdot 384 \\
1899 \cdot 320\end{array}$ & & & & $\begin{array}{l}129.4 \\
137.1\end{array}$ & $\begin{array}{l}\text { o. } 3 \mathrm{r} \\
\text { o. } 35\end{array}$ & & & & . & & \\
\hline $\begin{array}{r}1897.422 \\
1899.325 \\
.328 \\
.341 \\
\end{array}$ & $0 \Sigma 235 \ldots \ldots \ldots \ldots \ldots \ldots$ & II 264 I & +6138 & $\begin{array}{l}106.1 \\
118.7 \\
109.7 \\
114.8 \\
\end{array}$ & $\begin{array}{l}0.59 \\
0.60 \\
0.61 \\
0.66\end{array}$ & $\begin{array}{l}4,4 \\
4,2 \\
4,4 \\
4,4\end{array}$ & $\begin{array}{l}2 \\
2 \\
3 \\
2\end{array}$ & $\mid \begin{array}{cc}26-\text { inch } \\
“ & 11 \\
“ & \prime \prime\end{array}$ & $\begin{array}{l}600 \\
600 \\
888 \\
888\end{array}$ & $\begin{array}{l}\mathrm{Br} . \\
\mathrm{Br} . \\
\mathrm{Br} \text {. } \\
\mathrm{Br} .\end{array}$ & $\begin{array}{l}\text { Unsteady. } \\
\text { Very poor. }\end{array}$ \\
\hline $\begin{array}{l}1897.422 \\
1899 \cdot 331\end{array}$ & & & & $\begin{array}{l}106.1 \\
114.4\end{array}$ & $\begin{array}{l}0.59 \\
0.62\end{array}$ & & & & & & \\
\hline
\end{tabular}




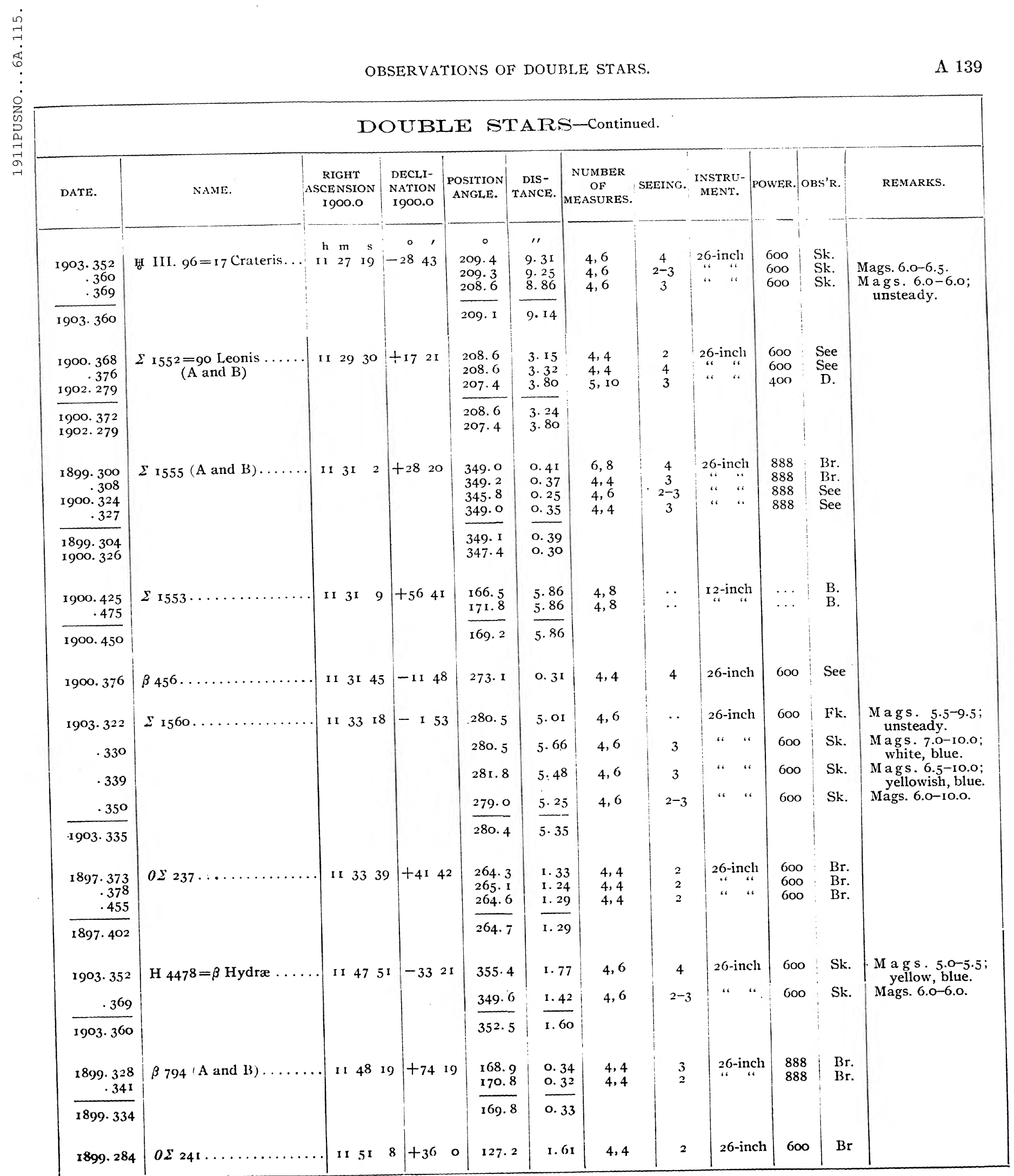




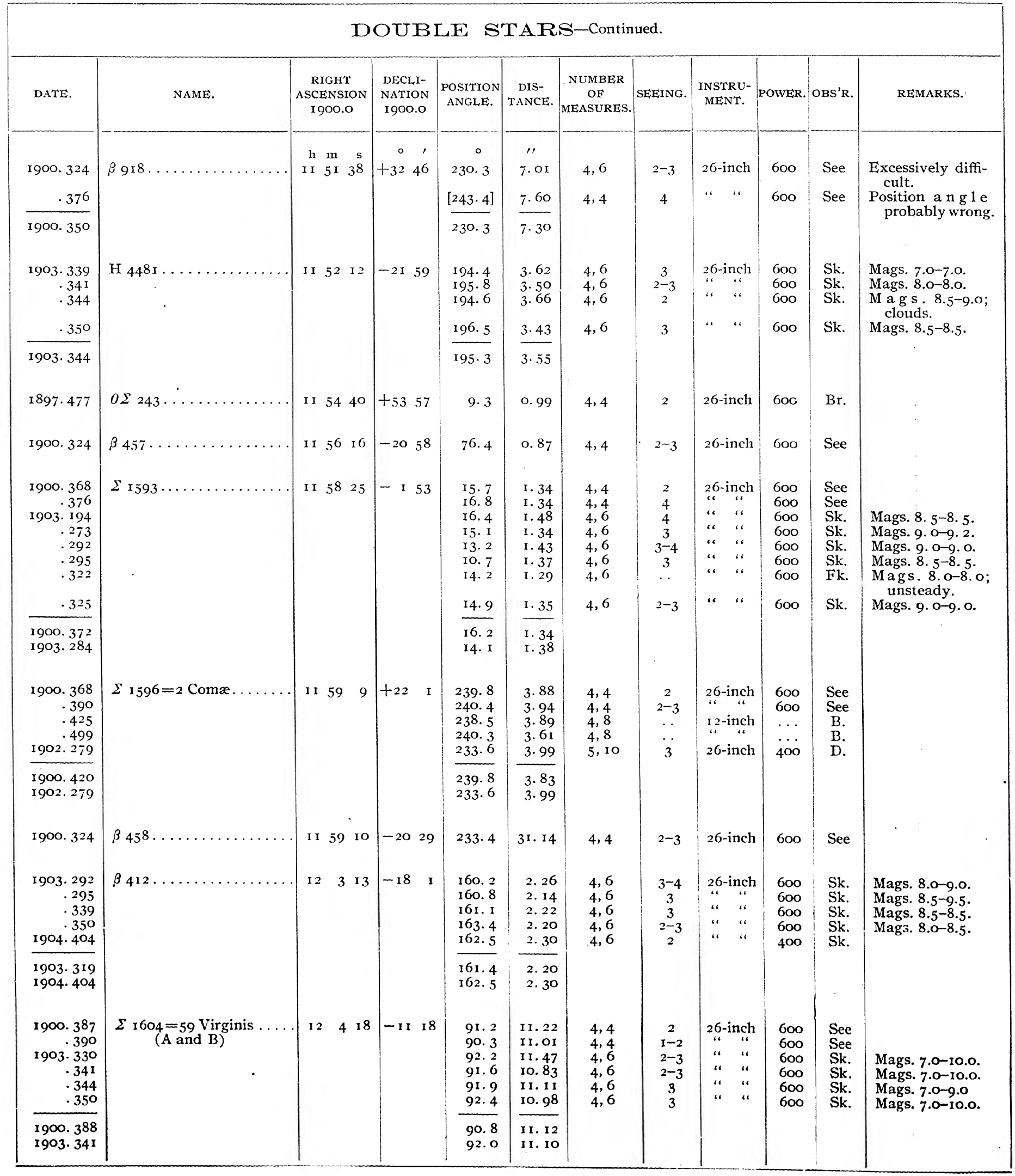


DOUBLE STARS-Continued.

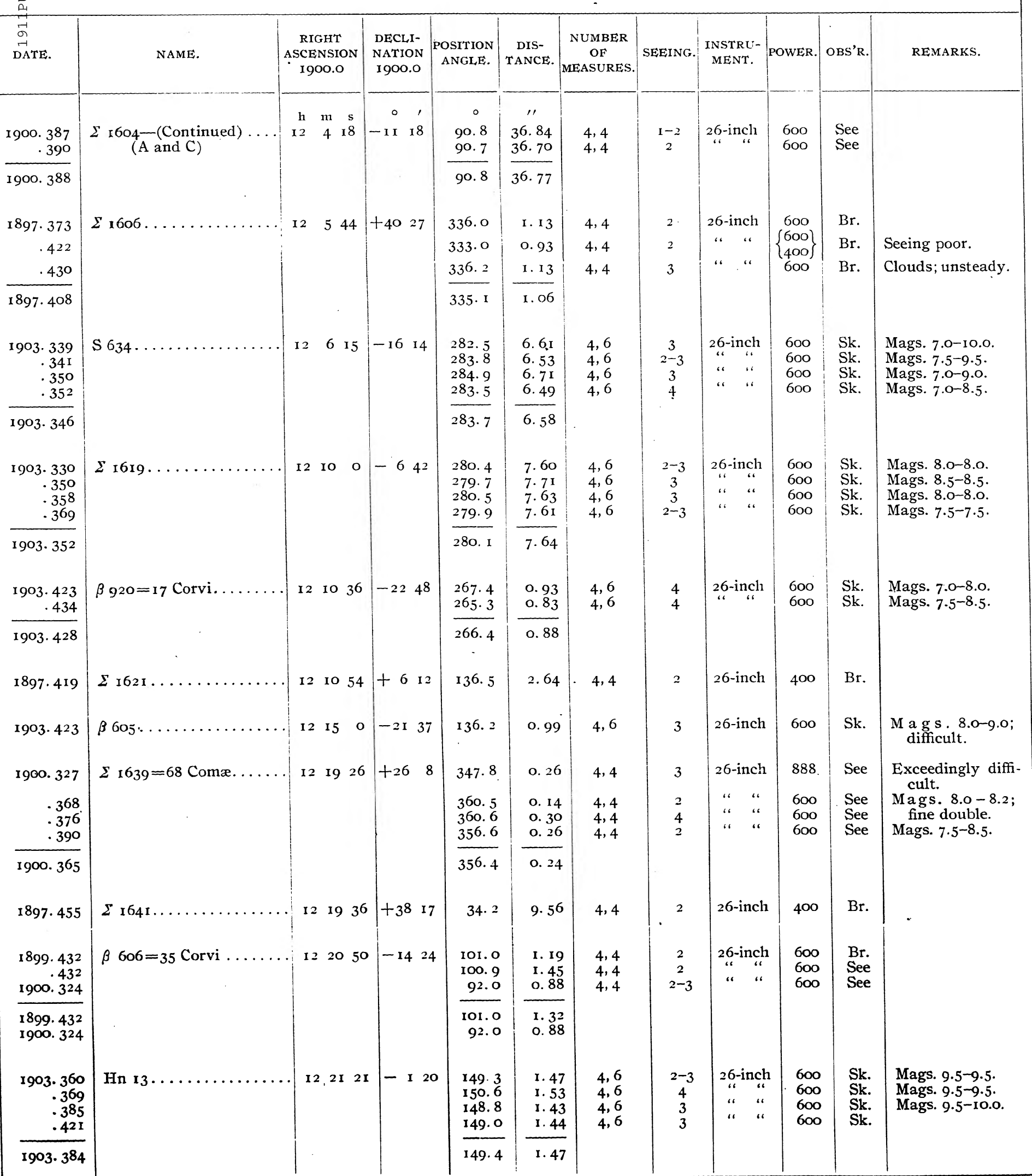




\begin{tabular}{|c|c|c|c|c|c|c|c|c|c|c|c|}
\hline \multicolumn{12}{|c|}{ DOUBLE STARS-Continued. } \\
\hline DATE. & NAME. & $\begin{array}{c}\text { RIGHT } \\
\text { ASCENSION } \\
1900.0\end{array}$ & $\begin{array}{c}\text { DECLI- } \\
\text { NATION } \\
\text { I } 900.0\end{array}$ & $\begin{array}{c}\text { POSITION } \\
\text { ANGLE. }\end{array}$ & $\begin{array}{c}\text { DIS- } \\
\text { TANCE. }\end{array}$ & $\begin{array}{c}\text { NUMBER } \\
\text { OF } \\
\text { MEASURES. }\end{array}$ & SEEING. & $\begin{array}{c}\text { INSTRL- } \\
\text { MENT. }\end{array}$ & POWER. & OBS'R. & REMARKS. \\
\hline $1897 \cdot 408$ & $\Sigma 1643 \ldots \ldots$ & $\begin{array}{ccc}\mathrm{h} & \mathrm{m} & \mathrm{s} \\
\mathrm{I} 2 & 22 & \mathrm{I} 3\end{array}$ & $\begin{array}{r}0 \\
+2735\end{array}$ & $\begin{array}{c}\circ \\
40.2\end{array}$ & $\begin{array}{c}\prime \prime \\
\mathrm{x} .82\end{array}$ & 4,4 & 2 & 26-inch & 4.00 & Br. & \\
\hline $\begin{array}{r}1903.325 \\
.369 \\
.385 \\
.434\end{array}$ & $\beta 28 \ldots \ldots \ldots \ldots \ldots \ldots$ & I 22456 & -1250 & $\begin{array}{r}5.8 \\
13.7 \\
9.9 \\
10.0\end{array}$ & $\begin{array}{l}\text { I. } 63 \\
\text { I. } 63 \\
\text { I. } 45 \\
\text { I. } 67\end{array}$ & $\begin{array}{l}4,6 \\
4,6 \\
4,6 \\
4,6\end{array}$ & $\begin{array}{l}2-3 \\
3 \\
3 \\
3\end{array}$ & 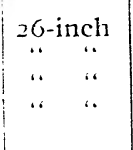 & $\begin{array}{l}600 \\
600 \\
600 \\
600\end{array}$ & $\begin{array}{l}\text { Sk. } \\
\text { Sk. } \\
\text { Sk. } \\
\text { Sk. }\end{array}$ & $\begin{array}{l}\text { Mags. 6.0-10.o. } \\
\text { Mags. 7.0-10.0. } \\
\text { Mags. 7.0-1 1.o. } \\
\text { Mags. 6.0-10.0. }\end{array}$ \\
\hline $1903 \cdot 378$ & & & & 9.8 & 1.60 & & & & & & \\
\hline $\begin{array}{r}1897.419 \\
\text { 1900. } 368 \\
.387\end{array}$ & $\Sigma 1647=191$ Virginis.... & I 22529 & tro 16 & $\begin{array}{l}220.9 \\
222.9 \\
223.9 \\
\end{array}$ & $\begin{array}{l}\text { I. } 29 \\
\text { I. } 62 \\
\text { I. } 54 \\
\end{array}$ & $\begin{array}{l}4,6 \\
4,4 \\
4,4\end{array}$ & $\begin{array}{l}2 \\
2 \\
2\end{array}$ & $\begin{array}{cc}26 \text {-inch } \\
" . & . " \\
" . & .\end{array}$ & $\begin{array}{l}400 \\
600 \\
600\end{array}$ & $\begin{array}{l}\text { Br. } \\
\text { See } \\
\text { See }\end{array}$ & \\
\hline $\begin{array}{l}1897 \cdot 419 \\
1900.378\end{array}$ & & & & $\begin{array}{l}220.9 \\
223.4\end{array}$ & $\begin{array}{l}\text { I. } 29 \\
\text { I. } 58\end{array}$ & & & & & & \\
\hline $\begin{array}{r}1899.432 \\
.432 \\
1900.324\end{array}$ & $\beta 797(A$ and $B) \ldots \ldots$ & I 22927 & $+63 \mathrm{I}$ & $\begin{array}{l}171.1 \\
171.2 \\
166.3\end{array}$ & $\begin{array}{l}0.73 \\
0.97 \\
0.60\end{array}$ & $\begin{array}{l}4,4 \\
4,4 \\
4,4\end{array}$ & $\begin{array}{c}2 \\
2 \\
2-3\end{array}$ & $\begin{array}{c}26-\text { inch } \\
" . " ، \\
“ "\end{array}$ & $\begin{array}{l}600 \\
600 \\
600\end{array}$ & $\begin{array}{l}\text { Br. } \\
\text { See } \\
\text { See }\end{array}$ & \\
\hline $\begin{array}{l}1899.432 \\
1900.324\end{array}$ & & & & $\begin{array}{l}\text { I } 71.2 \\
\text { I } 66.3\end{array}$ & $\begin{array}{l}0.85 \\
0.60\end{array}$ & & & & & & r \\
\hline 1900.324 & $(\mathrm{AB}$ and $\mathrm{C})$ & & ! & 3.0 & $77 \cdot 34$ & 4,4 & $2-3$ & 26-inch & 600 & See & 、 \\
\hline $\begin{array}{r}1897 \cdot 419 \\
1900.368 \\
.387 \\
\end{array}$ & $\Sigma 1658 \ldots \ldots \ldots \ldots \ldots$ & $1230 \quad 2$ & +80 & $\begin{array}{l}356.8 \\
357.2 \\
354.1\end{array}$ & $\begin{array}{l}2.58 \\
2.48 \\
2.42\end{array}$ & $\begin{array}{l}4,4 \\
4,4 \\
4,4\end{array}$ & $\begin{array}{c}2 \\
2 \\
2-1\end{array}$ & $\begin{array}{c}\text { 26-inch } \\
\text { “" } \\
\text { “" }\end{array}$ & $\begin{array}{l}400 \\
600 \\
600\end{array}$ & $\begin{array}{l}\text { Br. } \\
\text { See } \\
\text { See }\end{array}$ & \\
\hline $\begin{array}{l}1897.419 \\
1900.378\end{array}$ & & & ' & $\begin{array}{l}356.8 \\
355.6\end{array}$ & $\begin{array}{l}2.5^{8} \\
2.45\end{array}$ & & & & & & \\
\hline $\begin{array}{r}1897.419 \\
1900.387 \\
.390\end{array}$ & $\Sigma$ г66г............. & 123057 & t II 58 & $\begin{array}{l}236.7 \\
239.5 \\
236.8 \\
\end{array}$ & $\begin{array}{l}2.54 \\
2.37 \\
2.64\end{array}$ & $\begin{array}{l}4,4 \\
4,4 \\
4,4\end{array}$ & $\begin{array}{l}3 \\
2 \\
2\end{array}$ & 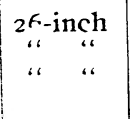 & $\begin{array}{l}400 \\
600 \\
600\end{array}$ & $\begin{array}{l}\text { Br. } \\
\text { See } \\
\text { See }\end{array}$ & \\
\hline $\begin{array}{l}\text { 1 } 897.419 \\
\text { 1900. } 388\end{array}$ & & & $:$ & $\begin{array}{l}236.7 \\
238.2\end{array}$ & $\begin{array}{l}2.54 \\
2.50\end{array}$ & & & & & & \\
\hline $\begin{array}{r}1897.419 \\
.444 \\
\end{array}$ & $\Sigma$ I $668=270$ Virginis $\ldots$ & I 23550 & +923 & $\begin{array}{l}194.6 \\
193.7 \\
\end{array}$ & $\begin{array}{l}\text { I. } 39 \\
\text { I. } 71 \\
\end{array}$ & $\begin{array}{l}4,4 \\
4,6\end{array}$ & $\begin{array}{l}3 \\
2\end{array}$ & 26-inch & $\begin{array}{l}400 \\
600\end{array}$ & $\begin{array}{l}\mathrm{Br} . \\
\mathrm{Br} .\end{array}$ & : \\
\hline $1897 \cdot 4.32$ & & & & I94. 2 & I. 55 & & & & & & \\
\hline $\begin{array}{r}1900.387 \\
.390 \\
1903.330 \\
.360 \\
.418 \\
.421 \\
.423 \\
\end{array}$ & $\Sigma{ }_{1669}=58$ Corvi $\ldots \ldots$ & $1236^{\circ} 4$ & -1228 & $\begin{array}{l}303.5 \\
305.8 \\
304.5 \\
306.1 \\
305.8 \\
305.2 \\
305.9 \\
\end{array}$ & $\begin{array}{l}5 \cdot 82 \\
5 \cdot 96 \\
5 \cdot 76 \\
5 \cdot 74 \\
5 \cdot 68 \\
5 \cdot 74 \\
5.89\end{array}$ & $\begin{array}{l}4,4 \\
4,4 \\
4,6 \\
4,6 \\
4,6 \\
4,6 \\
4,6\end{array}$ & $\begin{array}{c}1 \\
2 \\
2-3 \\
2-3 \\
2-3 \\
2-3 \\
4\end{array}$ & 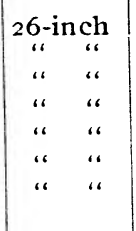 & $\begin{array}{l}600 \\
600 \\
600 \\
600 \\
600 \\
600 \\
600\end{array}$ & $\begin{array}{l}\text { See } \\
\text { See } \\
\text { Sk. } \\
\text { Sk. } \\
\text { Sk. } \\
\text { Sk. } \\
\text { Sk. }\end{array}$ & $\begin{array}{l}\text { Too unsteady to } \\
\text { continue. } \\
\text { Mags. 6.o-6.o. } \\
\text { Mags. } 5 \cdot 5-5 \cdot 5 \text {. } \\
\text { Mags. 6.0-6.o. }\end{array}$ \\
\hline $\begin{array}{l}1900.388 \\
1903 \cdot 390\end{array}$ & & & & $\begin{array}{l}304.6 \\
305.5\end{array}$ & $\begin{array}{l}5.89 \\
5 \cdot 76\end{array}$ & & & & & - & \\
\hline 1900.390 & $(A$ and $C)$ & & & $233 \cdot 7$ & 60.01 & 4,4 & 2 & 26-inch & 600 & See & Mag. of C' I r.o. \\
\hline
\end{tabular}


DOUBLE STARS-Continued.

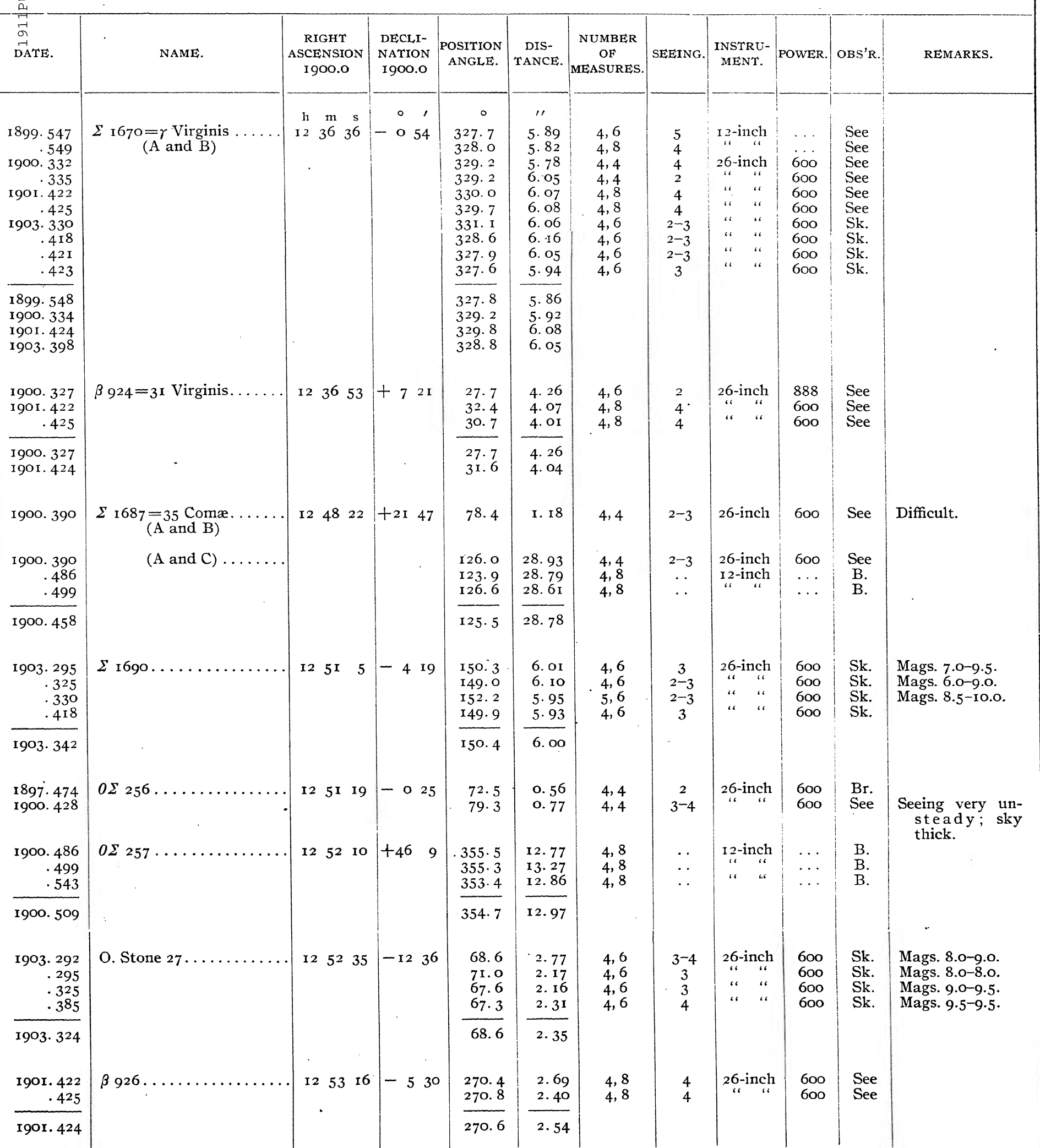




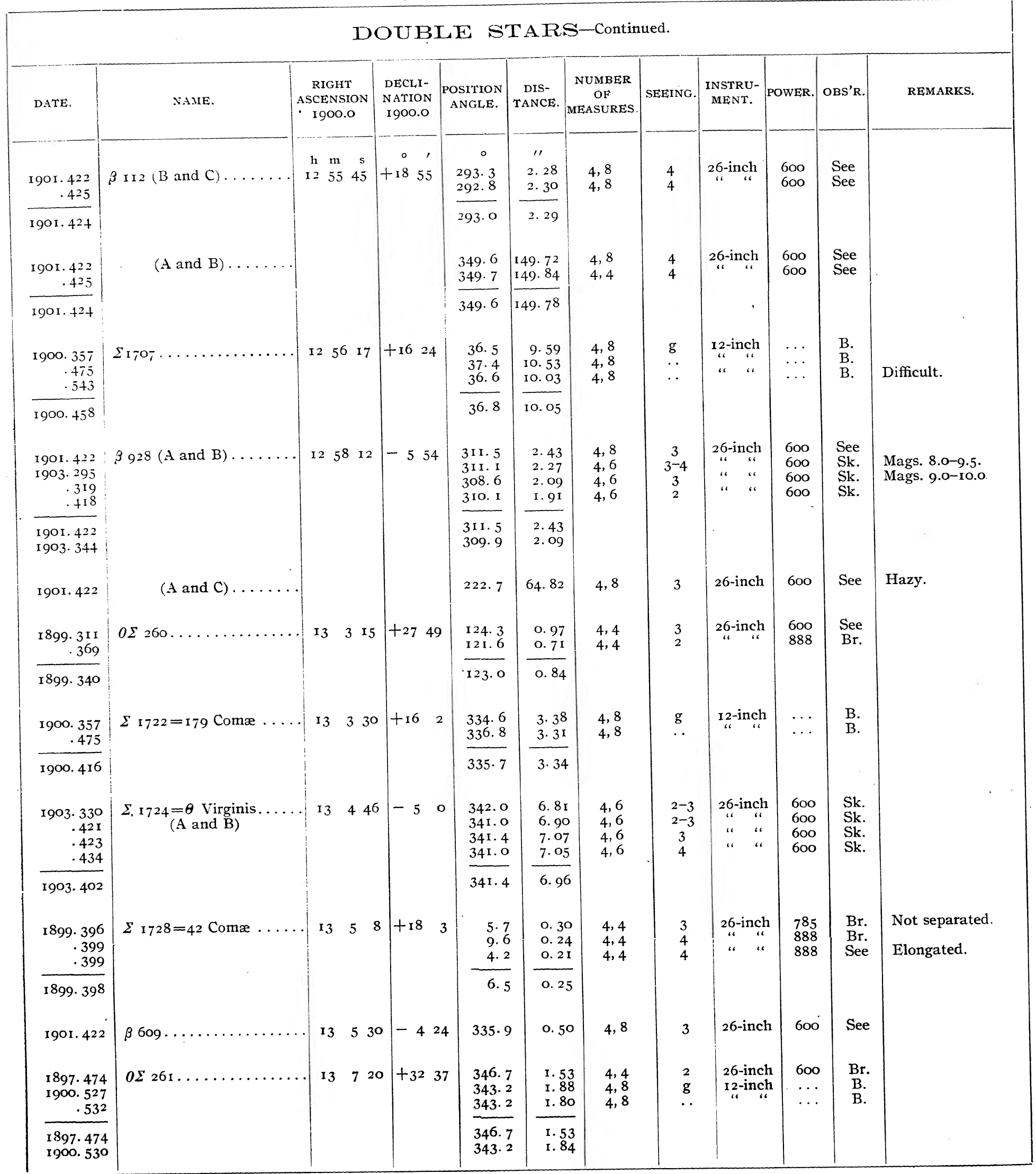




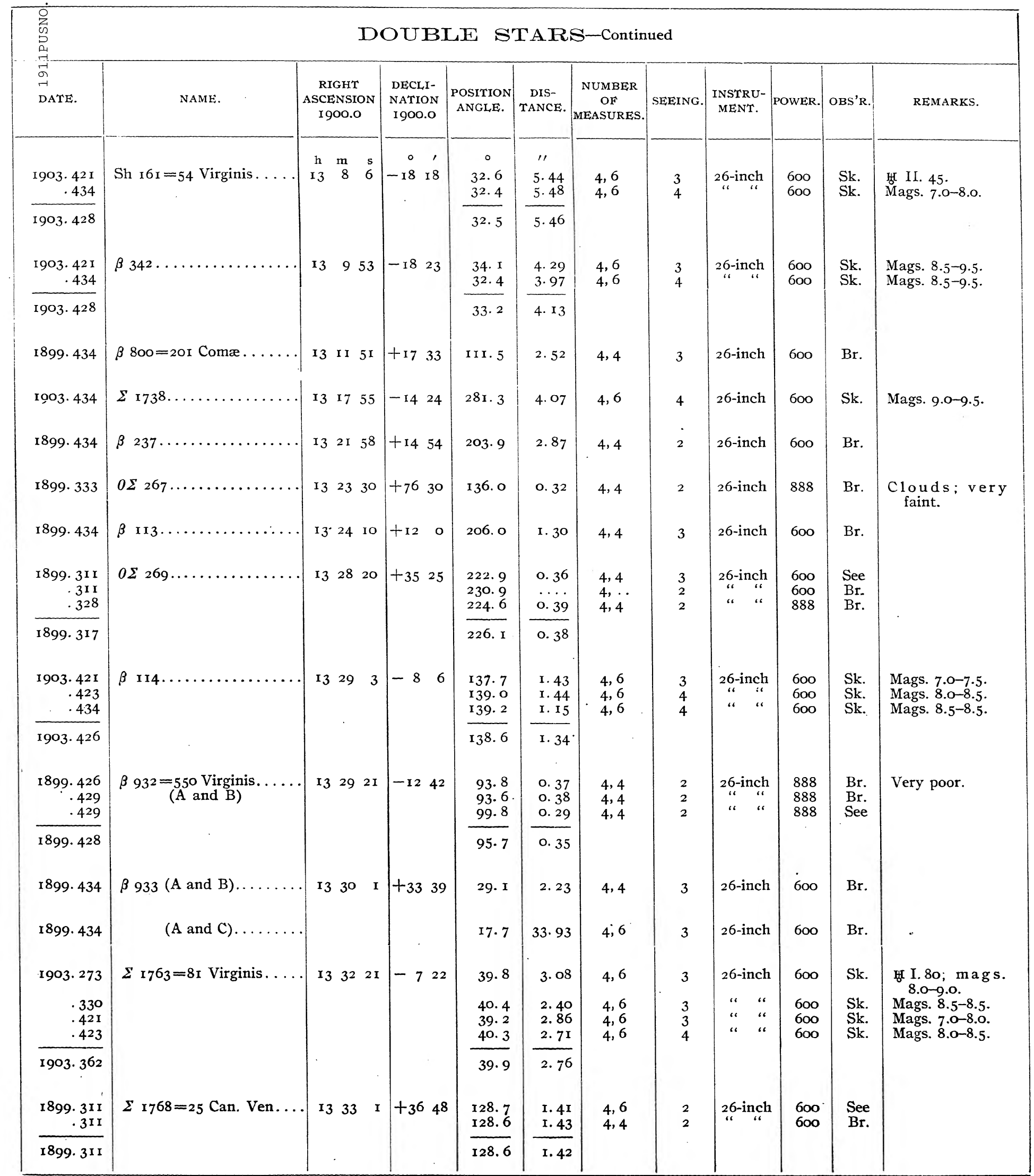

$28167^{\circ}$-VOL, 6-II - IO 


\begin{tabular}{|c|c|c|c|c|c|c|c|c|c|c|c|}
\hline \multicolumn{12}{|c|}{ DOUBLE STARS-Continued. } \\
\hline DATE. & NAME. & $\begin{array}{l}\text { RIGHT } \\
\text { ASCENSION } \\
\text { I900.0 }\end{array}$ & \begin{tabular}{c|c} 
DECLI- \\
$N$ & NATION \\
& I9OO.O
\end{tabular} & $\begin{array}{l}\text { POSITYON } \\
\text { ANGLE. }\end{array}$ & $\begin{array}{c}\text { DIS- } \\
\text { TANCE. }\end{array}$ & $\begin{array}{c}\text { NUMBER } \\
\text { OF } \\
\text { MEASURES }\end{array}$ & SEEING. & $\begin{array}{l}\text { INSTRU- } \\
\text { MENT. }\end{array}$ & POWER. & OBS'R. & REMARKS. \\
\hline $\begin{array}{r}1900.527 \\
.532 \\
\end{array}$ & $\Sigma$ І 77 ז $\ldots \ldots \ldots$ & $\begin{array}{rrr}\mathrm{h} & \mathrm{m} & \mathrm{s} \\
\mathrm{r} & 34 & 4\end{array}$ & 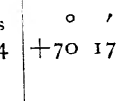 & $\begin{array}{c}\circ \\
71.8 \\
78.9 \\
\end{array}$ & $\begin{array}{l}\prime \prime \\
2.11 \\
2.24 \\
\end{array}$ & $\begin{array}{l}4,8 \\
4,8\end{array}$ & g & $\mid \begin{array}{l}\text { I2-inch } \\
،\end{array}$ & $\cdots$ & $\begin{array}{l}\text { B. } \\
\text { B. }\end{array}$ & \\
\hline x900. 530 & & & & $75 \cdot 4$ & 2. 18 & & & & & & \\
\hline $\begin{array}{r}1899 \cdot 341 \\
.369 \\
.369 \\
\end{array}$ & $\beta 612 \ldots$ & $1334 \quad 39$ & $9+\mathrm{II} \quad \mathrm{I} 5$ & $\begin{array}{l}220.6 \\
225.0 \\
232.9 \\
\end{array}$ & $\begin{array}{l}\text { o. } 35 \\
\text { o. } 32 \\
\text { o. } 29 \\
\end{array}$ & $\begin{array}{l}5,4 \\
4,4 \\
4,6\end{array}$ & $\begin{array}{l}2 \\
2 \\
2\end{array}$ & $\left|\begin{array}{cc}26-i n c h \\
، . \\
، & 4\end{array}\right|$ & $\begin{array}{l}888 \\
888 \\
888\end{array}$ & $\begin{array}{l}\text { Br. } \\
\text { Bir. } \\
\text { See }\end{array}$ & \\
\hline 1899.360 & & & & 226.2 & 0.32 & & . & & & & \\
\hline $\begin{array}{r}1900.527 \\
.532 \\
\end{array}$ & 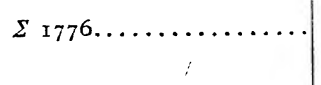 & 133740 & +4644 & $\begin{array}{l}199.2 \\
196.7\end{array}$ & $\begin{array}{l}7.16 \\
6.95\end{array}$ & $\begin{array}{l}4,8 \\
4,8\end{array}$ & $\mathrm{~g}$ & I 2 -inch & $\cdots$ & $\begin{array}{l}\text { B. } \\
\text { B. }\end{array}$ & \\
\hline 1900.530 & & & & 198.0 & 7.06 & & & & & & \\
\hline $\begin{array}{r}1897.466 \\
1899.369 \\
.369 \\
.388 \\
\end{array}$ & $\Sigma_{\mathrm{I} 78 \mathrm{r}} \ldots \ldots \ldots$ & I3 4 I 6 & $6+537$ & $\begin{array}{l}271.0 \\
268.2 \\
275.3 \\
272.9 \\
\end{array}$ & \begin{tabular}{l|} 
I. 00 \\
I. 43 \\
I. 08 \\
1.05 \\
\end{tabular} & $\begin{array}{l}4,4 \\
4,4 \\
5,4 \\
4,4\end{array}$ & $\begin{array}{l}2 \\
2 \\
2 \\
3\end{array}$ & $\begin{array}{cc}26-i n c h \\
" ، & ، \\
، & ، \\
، & ،\end{array}$ & $\begin{array}{l}600 \\
600 \\
600 \\
600\end{array}$ & $\begin{array}{l}\text { Br. } \\
\text { See } \\
\text { Br. } \\
\text { Br. }\end{array}$ & \\
\hline $\begin{array}{l}1897.466 \\
1899.375\end{array}$ & & & & $\begin{array}{l}271.0 \\
272.1\end{array}$ & $\begin{array}{l}\text { I. } 00 \\
\text { I. } 19\end{array}$ & & & & & & \\
\hline $\begin{array}{r}1897.466 \\
1900.527 \\
.532 \\
\end{array}$ & $\Sigma_{1785} \ldots \ldots \ldots \ldots$ & I3 443 I & I +2729 & $\begin{array}{l}266.3 \\
277 \cdot 1 \\
273 \cdot 9\end{array}$ & \begin{tabular}{l|} 
I. 45 \\
I. 94 \\
I. 97 \\
\end{tabular} & $\begin{array}{l}4,4 \\
4,8 \\
4,8\end{array}$ & $\begin{array}{l}2 \\
g \\
\cdots\end{array}$ & $\begin{array}{l}\text { 26-inch } \\
\text { 1 2-inch }\end{array}$ & $\begin{array}{l}600 \\
\cdots \\
\cdots\end{array}$ & $\begin{array}{l}\text { Br. } \\
\text { B. } \\
\text { B. }\end{array}$ & \\
\hline $\begin{array}{l}1897.466 \\
1900.530\end{array}$ & & & & $\begin{array}{l}266.3 \\
275.5\end{array}$ & $\begin{array}{l}\text { I. } 45 \\
\text { I. } 96\end{array}$ & & & & & & \\
\hline $1907 \cdot 435$ & Skinner $\left[\right.$ B. D. $\left.-15^{\circ} 3755\right]$ & I3 47 I2 & $2-1540$ & 344.6 & 3.63 & 4,6 & $\mathrm{p}$ & 26-inch & 600 & $\mathrm{R}$. & Mags. 8.8-10.7. \\
\hline $\begin{array}{r}1900.267 \\
.299\end{array}$ & Skinner $7 \ldots$ & 134846 & $6-1438$ & $\begin{array}{l}292.2 \\
297.1\end{array}$ & $\begin{array}{l}2.75 \\
2.13 \\
\end{array}$ & $\begin{array}{l}4,8 \\
4,8\end{array}$ & $\because$. & 1". & $\begin{array}{l}\cdots \\
\cdots\end{array}$ & $\begin{array}{l}\text { B. } \\
\text { B. }\end{array}$ & Very difficult. \\
\hline 1900.283 & & & & 294.6 & 2.44 & & & & & & \\
\hline $\begin{array}{r}1903.423 \\
.434 \\
.497 \\
.500 \\
\end{array}$ & $\Sigma_{\mathrm{I} 788}$. & 134943 & $3-734$ & $\begin{array}{l}80.5 \\
77.4 \\
79.9 \\
77.7 \\
\end{array}$ & $\begin{array}{l}2.84 \\
2.78 \\
2.68 \\
2.89 \\
\end{array}$ & $\begin{array}{l}4,6 \\
4,6 \\
4,6 \\
4,6\end{array}$ & $\begin{array}{l}4 \\
4 \\
2 \\
4\end{array}$ & $\mid \begin{array}{cc}26-i n c h \\
، \\
، & ، \\
، & ،\end{array}$ & $\begin{array}{l}600 \\
600 \\
600 \\
600\end{array}$ & $\begin{array}{l}\text { Sk. } \\
\text { Sk. } \\
\text { Sk. } \\
\text { Sk. }\end{array}$ & $\begin{array}{l}\text { Mags. 6.0 7.5. } \\
\text { Mags. 6.o-7.0. } \\
\text { Mags. 6.0 7.o. } \\
\text { Mags. 6.5 7.5. }\end{array}$ \\
\hline 1903.464 & & & & 78.9 & 2.80 & & & & & & \\
\hline 1897.474 & $0 \Sigma 273 \ldots$ & 135119 & $9+547$ & 109.2 & $0.9 \mathrm{I}$ & 4,4 & 2 & 26-inch & 600 & $\mathrm{Br}$. & $\begin{array}{l}\text { Distance some- } \\
\text { what uncer- } \\
\text { tain. }\end{array}$ \\
\hline $\begin{array}{r}1903.462 \\
.497 \\
\end{array}$ & $\Sigma \times 799 \ldots$ & 135934 & $34 \mid-6 \quad 4$ & $\begin{array}{r}295.8 \\
295.9 \\
\end{array}$ & $\begin{array}{l}4 \cdot 34 \\
4 \cdot 38 \\
\end{array}$ & $\begin{array}{l}4,6 \\
4,6\end{array}$ & $\begin{array}{c}4 \\
3-4\end{array}$ & "26-inch & $\begin{array}{l}600 \\
600\end{array}$ & $\begin{array}{l}\text { Sk. } \\
\text { Sk. }\end{array}$ & $\begin{array}{l}\text { tain. } \\
\text { Mags. 8.o-9.o. } \\
\text { Mags. 8.0-8.5. }\end{array}$ \\
\hline 1903.480 & & & . & 295.8 & $4 \cdot 36$ & & & & & & \\
\hline 1903.497 & $\Sigma 1802 \ldots$ & 14240 & $40-1227$ & $28 \mathrm{r} .8$ & 5. II & 4,6 & $3-4$ & 26-inch & 600 & Sk. & Mags. 8.0-9.o. \\
\hline
\end{tabular}




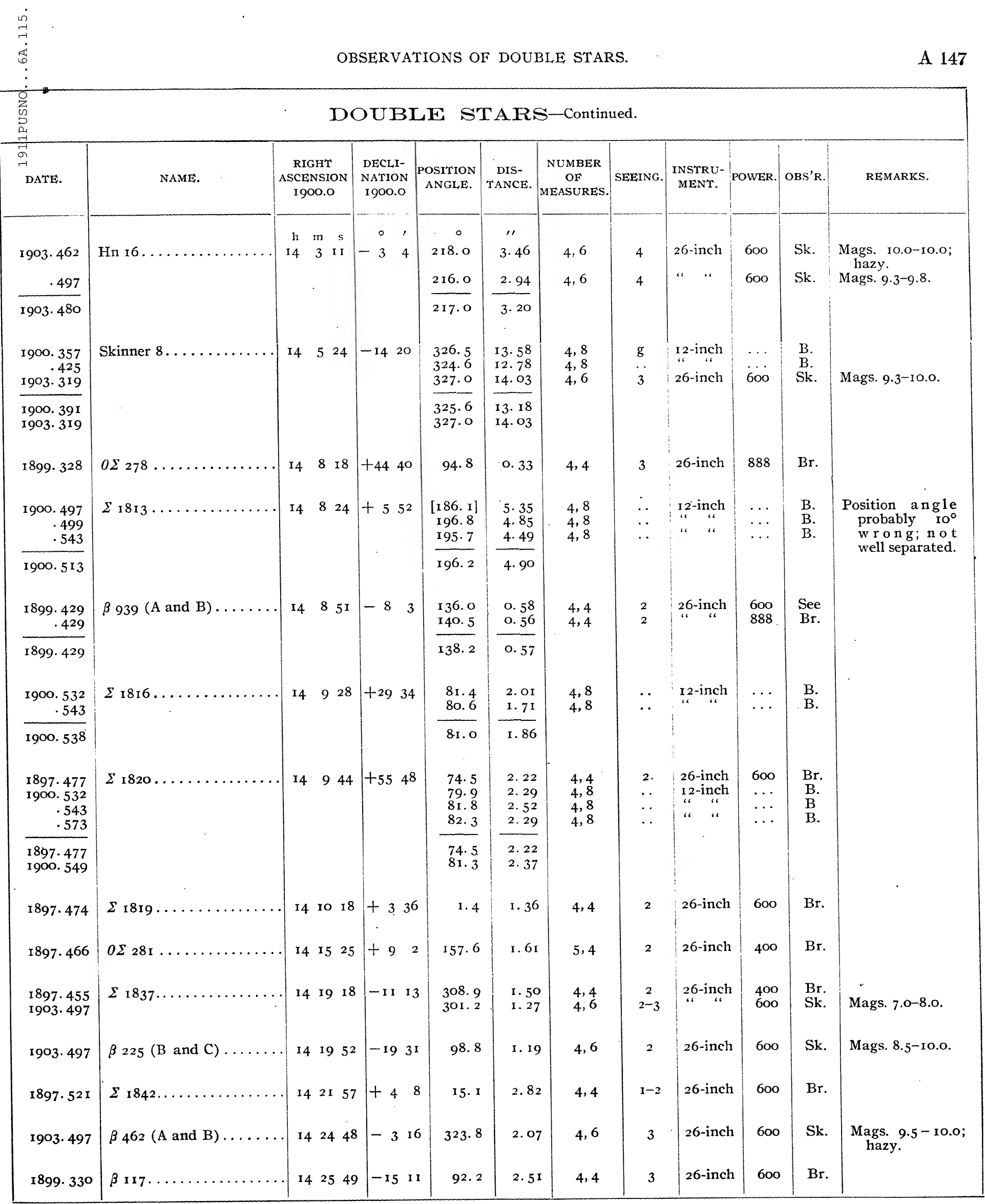




\begin{tabular}{|c|c|c|c|c|c|c|c|c|c|c|c|}
\hline \multicolumn{12}{|c|}{ DOUBLE STARS-Continued. } \\
\hline DATE. & NAME. & $\begin{array}{c}\text { RIGHT } \\
\text { ASCENSION } \\
1900.0\end{array}$ & $\begin{array}{c}\text { DECLI- } \\
\text { NATION } \\
\text { I9OO.O }\end{array}$ & $\begin{array}{c}\text { POSITION } \\
\text { ANGLE. }\end{array}$ & $\begin{array}{c}\text { DIS- } \\
\text { TANCE. }\end{array}$ & $\begin{array}{c}\text { NUMBER } \\
\text { OF } \\
\text { MEASURES. }\end{array}$ & SEEING. & $\begin{array}{c}\text { INSTRU- } \\
\text { MENT. }\end{array}$ & POWER. & OBS'R. & REMARKS. \\
\hline $\begin{array}{r}1900.497 \\
.527 \\
.573 \\
1901.499 \\
\end{array}$ & $\Sigma{ }_{1858} \ldots \ldots \ldots \ldots \ldots$ & $\begin{array}{rrr}\mathrm{h} & \mathrm{m} & \mathrm{s} \\
\mathrm{I} 4 & 29 & 30\end{array}$ & $\begin{array}{r}0 \\
+36 \\
\end{array}$ & $\begin{array}{c}\circ \\
44.2 \\
35.3 \\
39.4 \\
34.8\end{array}$ & $\begin{array}{l}\prime \prime \\
3.57 \\
3.02 \\
2.40 \\
2.66\end{array}$ & $\begin{array}{l}4,8 \\
4,8 \\
4,8 \\
4,8\end{array}$ & $\begin{array}{l}\mathrm{p} \\
\mathrm{g} \\
. \\
\cdots\end{array}$ & $\begin{array}{l}\text { I } 2 \text {-inch } \\
\text { "، } \\
\text { 26-inch }\end{array}$ & $\begin{array}{l}\cdots \\
\cdots \\
\cdots \\
400\end{array}$ & $\begin{array}{c}\text { B. } \\
\text { B. } \\
\text { B. } \\
\text { La. }\end{array}$ & \\
\hline $\begin{array}{l}1900.532 \\
1901.499\end{array}$ & & & & $\begin{array}{l}39.6 \\
34.8\end{array}$ & $\begin{array}{l}3.00 \\
2.66\end{array}$ & & & & & & \\
\hline $\begin{array}{r}1899.557 \\
1900.497 \\
.527 \\
\end{array}$ & $\Sigma_{\mathrm{I} 864}=\pi$ Bootis $\ldots$ & $1436 \quad 2$ & $+165 \mathrm{I}$ & $\begin{array}{l}\text { 104. } 8 \\
\text { IOI. } 2 \\
\text { I04. } \\
\end{array}$ & $\begin{array}{l}6.08 \\
6.59 \\
6.57 \\
\end{array}$ & $\begin{array}{l}8,16 \\
4,8 \\
4,8\end{array}$ & $\begin{array}{l}4 \\
\mathrm{p} \\
\mathrm{g}\end{array}$ & $\mid \begin{array}{cc}\text { 12-inch } \\
\text { “" } \\
\text { " }\end{array}$ & $\begin{array}{l}\cdots \\
\cdots \\
\cdots\end{array}$ & $\begin{array}{l}\text { See } \\
\text { B. } \\
\text { B. }\end{array}$ & \\
\hline $\begin{array}{l}899.557 \\
\text { I900. } 512\end{array}$ & & & & $\begin{array}{l}104.8 \\
102.6\end{array}$ & $\begin{array}{l}6.08 \\
6.5^{8}\end{array}$ & & & & & & \\
\hline I 899. 399 & $\Sigma{ }_{1865}=\zeta$ Bootis $\ldots \ldots$ & $14 \quad 3622$ & +149 & I6I. 4 & 0.22 & 3,4 & 3 & 26-inch & 888 & See & \\
\hline 1897.499 & $\Sigma \times 866 \ldots \ldots$ & $14365^{2}$ & +957 & 23.0 & 0.80 & 4,4 & $2-1$ & 26-inch & 600 & $\mathrm{Br}$. & $\begin{array}{l}\text { Very faint and } \\
\text { unsteady. }\end{array}$ \\
\hline $1901.44 \mathrm{I}$ & $\beta 807 \ldots$ & $14374 \mathrm{I}$ & -623 & 242.0 & I. 30 & 4,8 & 4 & 26-inch & 600 & See & \\
\hline $\begin{array}{r}1897.477 \\
1900.497 \\
.527 \\
\end{array}$ & $\Sigma{ }_{1871 \ldots \ldots \ldots \ldots \ldots}$ & 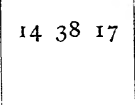 & +5150 & $\begin{array}{l}292.4 \\
289.2 \\
294.6 \\
\end{array}$ & $\begin{array}{l}\text { I. } 83 \\
2.39 \\
2.06 \\
\end{array}$ & $\begin{array}{l}4,4 \\
4,8 \\
4,8\end{array}$ & $\begin{array}{l}2 \\
\mathrm{p} \\
\mathrm{g}\end{array}$ & $\begin{array}{l}\text { 26-inch } \\
\text { I 2-inch }\end{array}$ & $\begin{array}{l}600 \\
\cdots \\
\cdots\end{array}$ & $\begin{array}{l}\mathrm{Br} . \\
\mathrm{B} . \\
\mathrm{B} .\end{array}$ & \\
\hline $\begin{array}{l}1897.477 \\
1900.512\end{array}$ & & & & \begin{tabular}{l|}
292.4 \\
291.9
\end{tabular} & $\begin{array}{l}1.83 \\
2.22\end{array}$ & & & & & & \\
\hline $\begin{array}{r}1899.544 \\
.547 \\
.557 \\
1900.617 \\
.650 \\
.658 \\
\end{array}$ & $\Sigma_{\mathrm{I} 877}=\varepsilon$ Bootis... & I4 4037 & +2730 & $\begin{array}{l}327.5 \\
327.6 \\
327.5 \\
326.3 \\
331.3 \\
323.1 \\
\end{array}$ & \begin{tabular}{l|}
3.63 \\
2.96 \\
2.80 \\
3.09 \\
2.97 \\
$3.4 \mathrm{I}$ \\
\end{tabular} & $\begin{array}{l}4,8 \\
4,6 \\
4,8 \\
4,8 \\
4,8 \\
4,8\end{array}$ & $\begin{array}{l}3 \\
5 \\
4 \\
\cdots \\
\cdots \\
\cdots\end{array}$ & 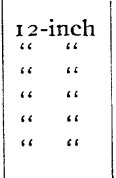 & $\begin{array}{l}\ldots \\
\cdots \\
\cdots \\
\cdots \\
\cdots\end{array}$ & $\begin{array}{l}\text { See } \\
\text { See } \\
\text { See } \\
\text { B. } \\
\text { B. } \\
\text { B. }\end{array}$ & \\
\hline $\begin{array}{l}1899.549 \\
1900.642\end{array}$ & & & & $\begin{array}{l}327.5 \\
326.9\end{array}$ & $\begin{array}{l}\text { 3. } 13 \\
3.16\end{array}$ & & & & & & \\
\hline $\begin{array}{l}1897 \cdot 521 \\
1903 \cdot 497\end{array}$ & $\Sigma_{1876 \ldots}$ & I4 $4 \mathrm{I} \quad 4$ & $-65^{8}$ & $\begin{array}{l}74 \cdot 4 \\
74 \cdot 2\end{array}$ & $\begin{array}{l}\text { I. } 45 \\
\text { I. } 27\end{array}$ & $\begin{array}{l}4,4 \\
4,6\end{array}$ & $\begin{array}{c}\mathrm{I}-2 \\
3\end{array}$ & "26-inch & $\begin{array}{l}600 \\
600\end{array}$ & $\begin{array}{l}\text { Br. } \\
\text { Sk. }\end{array}$ & Mags. 8.5-9.o. \\
\hline $\begin{array}{r}\mathrm{I} 897.5 \mathrm{IO} \\
.523 \\
\mathrm{I} 899.300 \\
.308 \\
.31 \mathrm{I} \\
.3 \mathrm{II} \\
.42 \mathrm{I} \\
\end{array}$ & $\Sigma$ I $879 \ldots$ & $\begin{array}{lll}14 & 4 & 2\end{array}$ & tro 5 & $\begin{array}{l}149.9 \\
148.0 \\
139.1 \\
140.2 \\
138.8 \\
\ldots \ldots . \\
138.2 \\
\end{array}$ & \begin{tabular}{l|} 
o. 44 \\
o. 59 \\
o. 48 \\
o. $5 \mathrm{O}$ \\
o. 66 \\
O. 46 \\
O. 40 \\
\end{tabular} & $\begin{array}{l}4,4 \\
4,4 \\
4,4 \\
4,1 \\
4,4 \\
., 4 \\
4,4\end{array}$ & $\begin{array}{l}2 \\
2 \\
3 \\
2 \\
3 \\
3 \\
2 \\
2\end{array}$ & 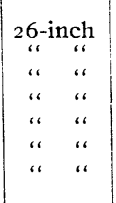 & $\begin{array}{l}600 \\
600 \\
888 \\
600 \\
600 \\
600 \\
888\end{array}$ & $\begin{array}{l}\mathrm{Br} . \\
\mathrm{Br} . \\
\mathrm{Br} . \\
\mathrm{Br} . \\
\text { See } \\
\mathrm{Br} . \\
\mathrm{Br} .\end{array}$ & \\
\hline $\begin{array}{l}1897.516 \\
1899.330\end{array}$ & . & & & $\begin{array}{l}\text { I49. } \\
\text { I39.1 }\end{array}$ & $\begin{array}{l}\text { o. } 52 \\
\text { o. } 5^{\circ}\end{array}$ & & & & & & \\
\hline $\begin{array}{r}1899.396 \\
.399 \\
\end{array}$ & $O \Sigma 285 \ldots$ & $144 \mathrm{r} 44$ & +4248 & $\begin{array}{l}137.5 \\
135.9 \\
\end{array}$ & $\begin{array}{l}\text { o. } 36 \\
\text { o. } 28 \\
\end{array}$ & $\begin{array}{l}4,4 \\
4,4\end{array}$ & $\begin{array}{l}3 \\
2\end{array}$ & $\underset{.1}{26-\text { inch }}$ & $\begin{array}{l}785 \\
888\end{array}$ & $\begin{array}{l}\mathrm{Br} . \\
\mathrm{Br}\end{array}$ & \\
\hline $1899 \cdot 398$ & & . & & 136.7 & 0.32 & & & & & & \\
\hline I90I. 44I & $\beta 346=23$ Libræ. & $\begin{array}{lll}14 & 42 & 57\end{array}$ & -1655 & $247 \cdot 5$ & I. 65 & 4,8 & 4 & 26-inch & 600 & See & \\
\hline
\end{tabular}




\begin{tabular}{|c|c|c|c|c|c|c|c|c|c|c|c|}
\hline 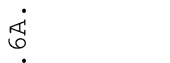 & \multicolumn{10}{|c|}{ OBSERVATIONS OF DOUBLE STARS. } & \multirow[t]{2}{*}{ A 149} \\
\hline \multicolumn{11}{|c|}{ DOUBIE STARS-Continued. } & \\
\hline$\stackrel{\text { ने }}{\rightarrow-1}$ DATE. & NAME. & $\begin{array}{l}\text { RIGHT } \\
\text { ASCENSION } \\
\text { I9O0.0 }\end{array}$ & $\begin{array}{l}\text { DECLI- } \\
\text { NATION } \\
\text { I9O0.0 }\end{array}$ & $\begin{array}{l}\text { POSITION } \\
\text { ANGLE. }\end{array}$ & $\begin{array}{c}\text { DIS- } \\
\text { TANCE. }\end{array}$ & $\begin{array}{c}\text { NUMBER } \\
\text { OF } \\
\text { MEASURES. }\end{array}$ & SEEING. & $\begin{array}{l}\text { INSTRU- } \\
\text { MENT. }\end{array}$ & POWER. & OBS'R. & REMARKS. \\
\hline $\begin{array}{l}1897.515 \\
1901.441\end{array}$ & $\Sigma 1883 \ldots$ & $\begin{array}{ccc}\mathrm{h} & \mathrm{m} & \mathrm{s} \\
\mathrm{I} 4 & 43 & 55\end{array}$ & $\begin{array}{r}01 \\
+622\end{array}$ & $\begin{array}{c}\circ \\
63.6 \\
66.4\end{array}$ & $\begin{array}{l}\prime \prime \\
\text { o. } 58 \\
\text { o. } 5^{2}\end{array}$ & $\begin{array}{l}4,4 \\
4,8\end{array}$ & $\begin{array}{l}2 \\
4\end{array}$ & 26-inch & $\begin{array}{l}600 \\
600\end{array}$ & $\begin{array}{l}\text { Br. } \\
\text { See }\end{array}$ & $\begin{array}{l}\text { Mags; a bout } \\
\text { equal; } \\
\text { divided. }\end{array}$ \\
\hline $1897 \cdot 589$ & $\Sigma$ I $884=286$ Bootis.... & $1443 \quad 5^{8}$ & +2447 & $55 \cdot 2$ & I. 69 & 4,4 & $I-2$ & 26-inch & 600 & $\mathrm{Br}$ & \\
\hline $\begin{array}{r}\mathrm{I} 897.466 \\
.474 \\
\mathrm{I} 899.412 \\
.421 \\
.423 \\
.429 \\
.429 \\
.432 \\
.544 \\
.549 \\
\mathrm{I} 901.592 \\
\mathrm{I} 902.525 \\
.525 \\
.525 \\
\end{array}$ & $\Sigma$ I $888=\xi$ Bootis . . & 144647 & +1931 & $\begin{array}{l}218.7 \\
219.3 \\
208.8 \\
208.6 \\
208.3 \\
205.2 \\
208.6 \\
209.5 \\
208.5 \\
209.6 \\
196.2 \\
191.3 \\
194.4 \\
191.5 \\
\end{array}$ & $\begin{array}{l}2.94 \\
2.90 \\
2.56 \\
2.72 \\
2.74 \\
3.02 \\
2.90 \\
2.98 \\
3.22 \\
3.30 \\
2.71 \\
2.74 \\
2.60 \\
2.72\end{array}$ & $\begin{array}{l}4,4 \\
4,4 \\
4,6 \\
6,4 \\
4,6 \\
4,8 \\
4,4 \\
8,8 \\
4,6 \\
4,8 \\
5,10 \\
5,10 \\
5,10 \\
5,10\end{array}$ & $\begin{array}{l}2 \\
2 \\
2 \\
3 \\
3 \\
3 \\
3 \\
2 \\
3 \\
\mathrm{I} \\
3 \\
4 \\
\mathrm{~g} \\
\mathrm{~g}\end{array}$ & 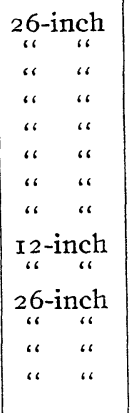 & $\begin{array}{l}400 \\
400 \\
600 \\
600 \\
600 \\
600 \\
600 \\
600 \\
\ldots . \\
\cdots \\
400 \\
600 \\
600 \\
600\end{array}$ & $\begin{array}{l}\text { Br. } \\
\text { Br. } \\
\text { Br. } \\
\text { Br. } \\
\text { Br. } \\
\text { See } \\
\text { Br. } \\
\text { See } \\
\text { See } \\
\text { See } \\
\text { See } \\
\text { See } \\
\text { Fk. } \\
\text { D. }\end{array}$ & $\begin{array}{l}\text { Clouds. } \\
\text { Companion red- } \\
\text { dish. } \\
\text { Companion red- } \\
\text { dish. } \\
\text { Clouds. }\end{array}$ \\
\hline $\begin{array}{l}1897 \cdot 470 \\
1899 \cdot 455 \\
1901.592 \\
1902.525\end{array}$ & & & . & $\begin{array}{l}219.0 \\
208.4 \\
196.2 \\
192.4\end{array}$ & $\begin{array}{l}2.92 \\
2.93 \\
2.71 \\
2.69\end{array}$ & & & & & & . \\
\hline $\mathbf{1 8 9 7 \cdot 4 7 7}$ & $0 \Sigma 287 \ldots \ldots$ & I4 $475 \mathrm{I}$ & +4520 & 138.7 & 0.68 & 4,4 & 2 & 26-inch & 600 & Br. & \\
\hline $\begin{array}{r}1897.499 \\
1900.532 \\
.573 \\
.612 \\
\end{array}$ & $0 \Sigma 288 \ldots \ldots \ldots$ & I 44842 & $+\mathrm{I} 67$ & $\begin{array}{l}\text { I } 89.6 \\
\text { I } 89.1 \\
\text { I } 94.8 \\
\text { I } 94.2 \\
\end{array}$ & $\begin{array}{l}\text { I. } 64 \\
2.22 \\
\text { I. } 35 \\
\text { I. } 69 \\
\end{array}$ & $\begin{array}{l}4,4 \\
4,8 \\
4,8 \\
4,8\end{array}$ & $\begin{array}{l}2 \\
\cdots \\
\cdots \\
\cdots\end{array}$ & $\begin{array}{c}\text { 26-inch } \\
\text { 1 } 2 \text {-inch } \\
\text { "، }\end{array}$ & $\begin{array}{l}600 \\
\cdots \\
\cdots \\
\cdots\end{array}$ & $\begin{array}{l}\mathrm{Br} . \\
\mathrm{B} . \\
\mathrm{B} . \\
\mathrm{B} .\end{array}$ & $\begin{array}{l}\text { Seen quite well } \\
\text { not yet dark. } \\
\text { Fainter than sup- } \\
\text { posed. }\end{array}$ \\
\hline $\begin{array}{l}\text { I } 897.499 \\
\text { I900. } 572\end{array}$ & & & & $\begin{array}{l}189.6 \\
192.7\end{array}$ & $\begin{array}{l}\text { I. } 64 \\
\text { I. } 75\end{array}$ & & & & & & \\
\hline $1899 \cdot 557$ & $0 \Sigma 289 \ldots$ & I 4 5I 50 & +3242 & II 9.2 & 4.66 & 4,8 & 4 & I 2 -inch & $\cdots$ & See & \\
\hline เǵor. 44I & $\beta 239=59$ Hydræ $\ldots \ldots$ & 145244 & $-27 \quad 15$ & 313. I & 1.08 & 4,8 & 4 & 26-inch & 600 & See & $\begin{array}{l}\text { M a gs. 6.0 } 7.0 \\
\text { divided at best } \\
\text { moments. }\end{array}$ \\
\hline I9OI. 44I & $\beta 808(A$ and $B) \ldots \ldots$ & I4 $525 \mathrm{I}$ & $-8 \mathrm{I} 7$ & I 89.6 & $0.5^{2}$ & 4,8 & 4 & 26-inch & 600 & See & Mags. IO.O-IO.O. \\
\hline I9OI. $44 \mathrm{I}$ & $(\mathrm{AB}$ and $\mathrm{C})$ & & & $305 \cdot 5$ & $93 \cdot 77$ & 4,8 & 4 & 26-inch & 600 & See & \\
\hline $\begin{array}{r}\mathrm{r} 899.300 \\
.388 \\
\end{array}$ & $\beta 348=2$ Serpentis. . & I4 5642 & to I5 & $\begin{array}{l}\text { II9.2. } \\
\mathbf{I 2 2 . 3} \\
\end{array}$ & $\begin{array}{l}\text { o. } 59 \\
\text { o. } 75\end{array}$ & $\begin{array}{l}4,4 \\
5,4\end{array}$ & $\begin{array}{l}2 \\
2\end{array}$ & $\underset{\text { 26-inch }}{ }$ & $\begin{array}{l}888 \\
600\end{array}$ & $\begin{array}{l}\mathrm{Br} . \\
\mathrm{Br} \text {. }\end{array}$ & \\
\hline I 899.344 & & & & I20. 8 & o. 67 & & & & & & \\
\hline $\begin{array}{r}\mathrm{x} 903.514 \\
.517 \\
.519 \\
\end{array}$ & 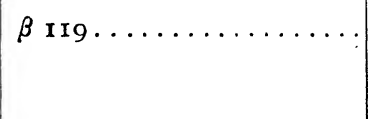 & $15 \quad 0 \quad 14$ & $-63^{8}$ & $\begin{array}{l}301.8 \\
302.0 \\
302.0 \\
\end{array}$ & $\begin{array}{l}\text { I. } 89 \\
\text { I. } 70 \\
\text { I. } 7 \mathrm{I} \\
\end{array}$ & $\begin{array}{l}4,6 \\
4,6 \\
4,6\end{array}$ & $\begin{array}{l}3 \\
3 \\
3\end{array}$ & $\begin{array}{cc}26-i n c h \\
\text { ، } \\
\text { " }\end{array}$ & $\begin{array}{l}600 \\
600 \\
600\end{array}$ & $\begin{array}{l}\text { Sk. } \\
\text { Sk. } \\
\text { Sk. }\end{array}$ & $\begin{array}{l}\text { Mags. } 7.5-8.0 . \\
\text { Mags. } 8.0-8.5 . \\
\text { Mags. } 8.0-8.5\end{array}$ \\
\hline 1903.517 & & & . & 301.9 & I. 77 & & & & & & \\
\hline
\end{tabular}




\begin{tabular}{|c|c|c|c|c|c|c|c|c|c|c|c|}
\hline \multicolumn{12}{|c|}{ DOUBLE STARS-Continued. } \\
\hline DATE. & NAME. & $\begin{array}{c}\text { RIGHT } \\
\text { ASCENSIO } \\
\text { I900.0 }\end{array}$ & $\begin{array}{c}\text { DECLI- } \\
\text { NATION } \\
\text { I } 900.0\end{array}$ & POSITION & $\begin{array}{c}\text { DIS- } \\
\text { TANCE. }\end{array}$ & $\begin{array}{c}\text { NUMBER } \\
\text { OF } \\
\text { MEASURES. }\end{array}$ & SEEING. & $\begin{array}{l}\text { INSTRU- } \\
\text { MENT. }\end{array}$ & POWER & OBS'R. & REMARKS. \\
\hline $\begin{array}{r}1899.547 \\
.557 \\
1900.527 \\
.532 \\
.590 \\
\end{array}$ & $\Sigma$ I909 $=44$ Bootis. & $\begin{array}{rrrr}h & m & s \\
15 & 0 & 30\end{array}$ & $\begin{array}{r}0 \\
+48 \\
0\end{array}$ & $\begin{array}{c}\circ \\
244.0 \\
243.4 \\
244.8 \\
236.9 \\
247.1 \\
\end{array}$ & $\begin{array}{l}\prime \prime \\
4.78 \\
4.74 \\
4.69 \\
4.94 \\
4.44 \\
\end{array}$ & $\begin{array}{l}4,6 \\
8,16 \\
4,8 \\
4,8 \\
4,8\end{array}$ & $\begin{array}{l}5 \\
4 \\
\mathrm{~g} \\
. \\
\cdots\end{array}$ & \begin{tabular}{cc|} 
I 2-inch \\
$،$ & “ \\
$،$ & $،$ \\
$،$ & $،$ \\
$،$ & $،$
\end{tabular} & $\begin{array}{l}\cdots \\
\cdots \\
\cdots \\
\cdots\end{array}$ & $\begin{array}{l}\text { See } \\
\text { See } \\
\text { B. } \\
\text { B. } \\
\text { B. }\end{array}$ & \\
\hline $\begin{array}{l}1899 \cdot 552 \\
1900.550\end{array}$ & & & & $\begin{array}{l}243.7 \\
242.9\end{array}$ & $\begin{array}{l}4.76 \\
4.69\end{array}$ & & & & & & \\
\hline $1897 \cdot 52 \mathrm{I}$ & $\Sigma 1908 \ldots \ldots$ & $15 \quad 0 \quad 55$ & $5+345^{I}$ & 148.8 & I. 4 I & 4,4 & $1-2$ & 26-inch & 600 & $\mathrm{Br}$. & \\
\hline I899. 557 & $\Sigma 1910 \ldots$. & $15 \quad 243$ & $3 \mid+937$ & 210.7 & 4. 49 & 4,8 & 4 & I 2 -inch & .. & See & \\
\hline $\begin{array}{r}1903.514 \\
.517 \\
.519 \\
\end{array}$ & $\Sigma_{3090} \ldots \ldots \ldots \ldots$ & $15 \quad 334$ & $4-036$ & $\begin{array}{l}278.0 \\
280.8 \\
278.9 \\
\end{array}$ & $\begin{array}{l}\text { I. } 5 \text { I } \\
\text { I. } 43 \\
\text { I. } 54 \\
\end{array}$ & $\begin{array}{l}4,6 \\
4,6 \\
4,6\end{array}$ & $\begin{array}{l}4 \\
4 \\
4\end{array}$ & $\begin{array}{c}\text { 26-inch } \\
\text { ". ". } \\
\text { " }\end{array}$ & $\begin{array}{l}600 \\
600 \\
600\end{array}$ & $\begin{array}{l}\text { Sk. } \\
\text { Sk. } \\
\text { Sk. }\end{array}$ & $\begin{array}{l}\text { Mags. 9.2-9.6. } \\
\text { Mags. 9.0-9.o. } \\
\text { Mags. 9.0-9.0. }\end{array}$ \\
\hline 1903.517 & & & & 279.2 & I. 49 & & & & & & \\
\hline I90I. $44 \mathrm{I}$ & $\beta 349 \ldots$ & $15 \quad 353$ & $3+24$ & 38.5 & 4.07 & 4,8 & $2-4$ & 26-inch & $600-$ & See & $\begin{array}{l}\text { Seeing getting } \\
\text { worse. }\end{array}$ \\
\hline $1903.5^{14}$ & $\beta 809 \ldots \ldots$ & $\mathrm{I}_{5} \quad 4 \quad 13$ & $3-2221$ & $123 \cdot 7$ & I. 99 & 4,6 & $3-4$ & 26-inch & 600 & Sk. & Mags. 8.5-10.o. \\
\hline $1903 \cdot 514$ & $\beta 350 \ldots$ & $\begin{array}{lll}15 & 9 & 4\end{array}$ & $\begin{array}{lll}-27 & 14\end{array}$ & 148.5 & I. 18 & 4,6 & 3 & 26-inch & 600 & Sk. & Mags. 8.o-8.5. \\
\hline $\mathrm{I} 897 \cdot 52 \mathrm{I}$ & $O \Sigma 294 \ldots$ & $\begin{array}{lll}15 & 10 \quad 19\end{array}$ & $9+56=25$ & 246.4 & 3.64 & 4,4 & $1-2$ & 26-inch & 600 & $\mathrm{Br}$. & \\
\hline I $899 \cdot 557$ & $\Sigma 1926 \ldots$ & 15119 & $9+3840$ & 254.7 & 0.94 & 4,8 & 4 & I 2 -inch & $\cdots$ & See & \\
\hline $\begin{array}{r}1903.5^{14} \\
.519 \\
\end{array}$ & $\Sigma 1925 \ldots$ & $15 \quad 1132$ & $2-755$ & $\begin{array}{l}12.4 \\
12.5 \\
\end{array}$ & $\begin{array}{l}5.11 \\
5.14 \\
\end{array}$ & $\begin{array}{l}4,6 \\
4,6\end{array}$ & $3-4$ & "1"inch & $\begin{array}{l}600 \\
600\end{array}$ & $\begin{array}{l}\text { Sk. } \\
\text { Sk. }\end{array}$ & $\begin{array}{l}\text { Mags. 8.5-9.5. } \\
\text { Mags. 8.5-10.0. }\end{array}$ \\
\hline 1903.516 & & & & 12.4 & 5.12 & & & & & & \\
\hline $\begin{array}{r}1903.514 \\
.519 \\
\end{array}$ & $\beta 227 \ldots \ldots$ & $\begin{array}{lll}15 & 13 & 17\end{array}$ & $7 \mid-2354$ & $\begin{array}{r}178.2 \\
177.2 \\
\end{array}$ & $\begin{array}{l}1.87 \\
2.33 \\
\end{array}$ & $\begin{array}{l}4,6 \\
4,6\end{array}$ & $\begin{array}{l}3 \\
3\end{array}$ & 26-inch & $\begin{array}{l}600 \\
600\end{array}$ & $\begin{array}{l}\text { Sk. } \\
\text { Sk. }\end{array}$ & $\begin{array}{l}\text { Mags. 8.0-8.5. } \\
\text { Mags. 8.0-9.5. }\end{array}$ \\
\hline$\overline{1903 \cdot 5 \times 6}$ & & & & $177 \cdot 7$ & 2. 10 & & & & & & \\
\hline 1903.519 & $\beta 228 \ldots$ & $\begin{array}{lll}15 & 1348\end{array}$ & \begin{tabular}{l|ll}
8 & -23 & 54
\end{tabular} & 320.3 & I. 02 & 4,6 & 3 & 26-inch & 600 & Sk. & Mags. $8.5-8.5$ \\
\hline $\begin{array}{r}1900.527 \\
.532 \\
\end{array}$ & $\Sigma \times 934 \ldots$ & $\begin{array}{lll}15 & 13 & 53\end{array}$ & $3+44$ 10 & $\begin{array}{l}29.7 \\
29.1 \\
\end{array}$ & $\begin{array}{l}6.80 \\
6.73 \\
\end{array}$ & $\begin{array}{l}4,8 \\
4,8\end{array}$ & $\mathrm{~g}$ & I"-inch & $\begin{array}{l}\cdots \\
\cdots\end{array}$ & $\begin{array}{l}\text { B. } \\
\text { B. }\end{array}$ & . \\
\hline 1900. 530 & & & & $29 \cdot 4$ & 6.76 & & & & & & \\
\hline $\begin{array}{r}1897.559 \\
.581 \\
\end{array}$ & $\Sigma 1932=1$ Coronæ & $1514 \quad 4$ & $4+27 \times 2$ & $\begin{array}{r}326.8 \\
327.7 \\
\end{array}$ & $\begin{array}{l}0.90 \\
0.84 \\
\end{array}$ & $\begin{array}{l}4,4 \\
4,4\end{array}$ & $\begin{array}{l}2 \\
3\end{array}$ & 26-inch & $\begin{array}{l}600 \\
600\end{array}$ & $\begin{array}{l}\mathrm{Br} . \\
\mathrm{Br} .\end{array}$ & \\
\hline 1897.570 & & & & 327.2 & 0.87 & & & & & & \\
\hline 1897.581 & $\Sigma{ }_{1937}=\eta$ Coronæ & 15194 & $4+3039$ & 336.5 & 0.49 & 4,4 & 3 & 26-inch & 888 & $\mathrm{Br}$ & \\
\hline
\end{tabular}




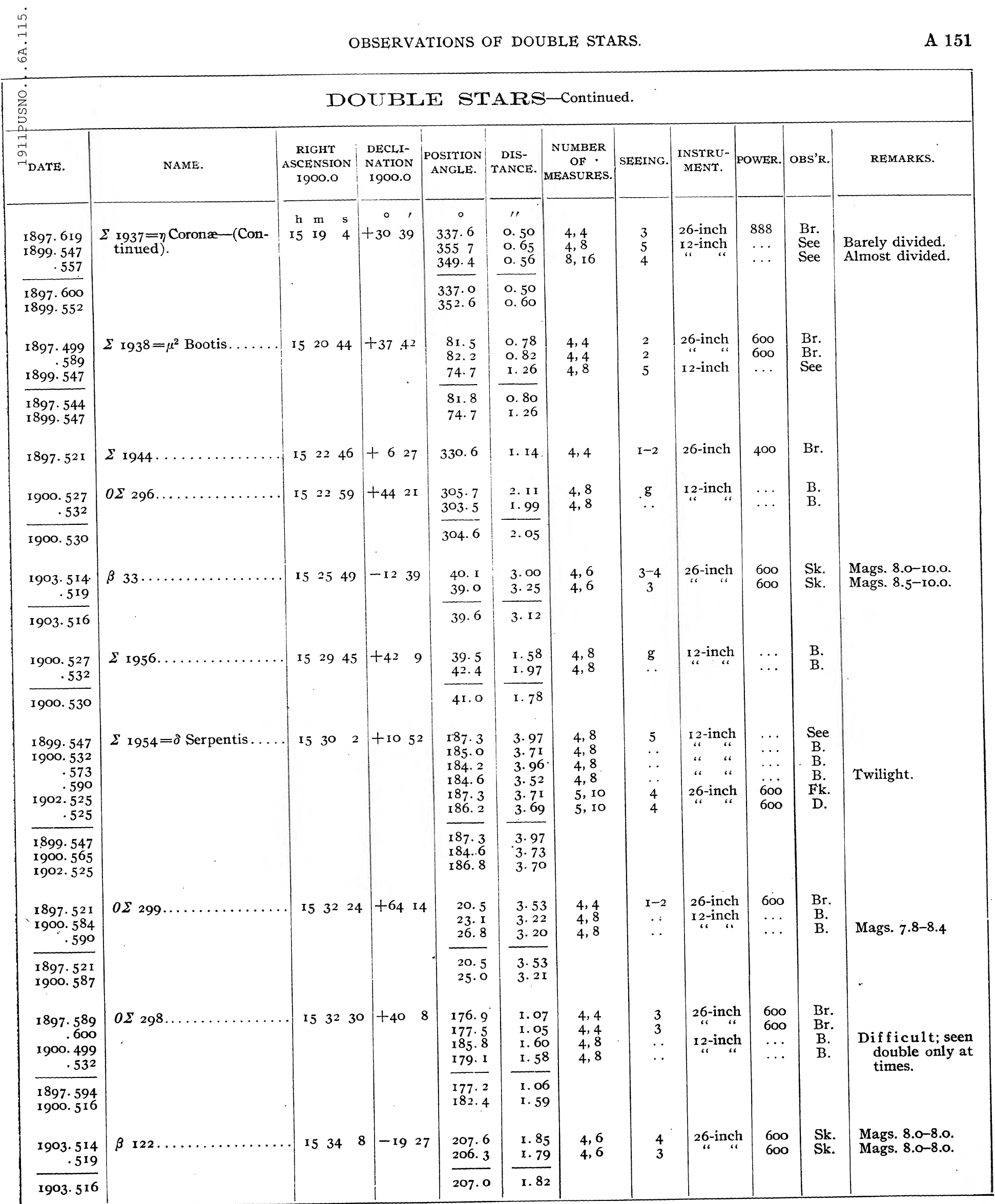




\begin{tabular}{|c|c|c|c|c|c|c|c|c|c|c|c|}
\hline \multicolumn{12}{|c|}{ DOUBLE STARS-Continued. } \\
\hline DATE. & NAME. & $\begin{array}{c}\text { RIGHT } \\
\text { ASCENSION } \\
\text { I } 900.0\end{array}$ & $\begin{array}{c}\text { DECLI- } \\
\text { NATION } \\
\text { I9OO.O }\end{array}$ & $\begin{array}{l}\text { POSITION } \\
\text { ANGLE. }\end{array}$ & $\begin{array}{c}\text { DIS- } \\
\text { TANCE. }\end{array}$ & \begin{tabular}{|l} 
NUMBER \\
OF \\
MEASURES.
\end{tabular} & SEEING. & $\begin{array}{l}\text { INSTRU- } \\
\text { MENT. }\end{array}$ & POWER. & OBS'R. & REMARKS. \\
\hline 1907.517 & Howe $37 \ldots$ & $\begin{array}{rrr}\text { h } & \text { m } & \text { s } \\
15 & 35 & 26\end{array}$ & $\mid \begin{array}{rr}0 & 1 \\
-14 & 30\end{array}$ & $\begin{array}{l}\circ \\
89.9\end{array}$ & $5 \cdot 52$ & 4,6 & $f-g$ & 26-inch & 400 & R. & Mags. 9.o-9.o. \\
\hline $\begin{array}{r}1900.584 \\
.590 \\
.658 \\
\end{array}$ & $\Sigma{ }_{1965}=\zeta$ Coronæ........ & 153537 & +3658 & $\begin{array}{l}305 \cdot 2 \\
304 \cdot 3 \\
304.6 \\
\end{array}$ & $\begin{array}{l}6.50 \\
6.39 \\
6.43 \\
\end{array}$ & $\begin{array}{l}4,8 \\
4,8 \\
4,8\end{array}$ & $\begin{array}{l}\cdots \\
\cdots \\
\cdots\end{array}$ & $\begin{array}{c}\text { I 2-inch } \\
\text { ". } \\
" . "\end{array}$ & $\begin{array}{l}\cdots \\
\cdots \\
\cdots\end{array}$ & $\begin{array}{l}\text { B. } \\
\text { B. } \\
\text { B. }\end{array}$ & \\
\hline $1900.6 \mathrm{II}$ & & & & $304 \cdot 7$ & 6.44 & & & & & & \\
\hline $\begin{array}{r}1903.514 \\
.519\end{array}$ & $\beta 35 \ldots \ldots \ldots \ldots$ & 15379 & -1542 & $\begin{array}{l}\text { 100. } 6 \\
\text { 10I. } 6\end{array}$ & $\begin{array}{l}2.50 \\
2.74\end{array}$ & $\begin{array}{l}4,6 \\
4,6\end{array}$ & $\begin{array}{c}3-4 \\
3\end{array}$ & $\underset{\text { "26-inch }}{4}$ & $\begin{array}{l}600 \\
600\end{array}$ & $\begin{array}{l}\text { Sk. } \\
\text { Sk. }\end{array}$ & $\begin{array}{l}\text { Mags. 8.0-10.0. } \\
\text { Mags. 7.0-9.5. }\end{array}$ \\
\hline 1903.516 & & & & IOI. I & 2.62 & & & & & & \\
\hline $\begin{array}{r}1897.616 \\
.619 \\
1899.547 \\
.582 \\
\end{array}$ & $\Sigma$ I967 $=r$ Coronæ.. & I 53833 & +2637 & $\begin{array}{l}120.3 \\
120.5 \\
116.0 \\
117.3 \\
\end{array}$ & $\begin{array}{l}0.60 \\
0.65 \\
\text { I. } 14 \\
0.60 \\
\end{array}$ & $\begin{array}{l}4,4 \\
4,4 \\
4,8 \\
8,14\end{array}$ & $\begin{array}{c}3 \\
2-3 \\
5 \\
5\end{array}$ & $\begin{array}{l}\text { 26-inch } \\
\text { "i" " } \\
\text { I 2-inch } \\
\text { " " }\end{array}$ & $\begin{array}{l}888 \\
600 \\
\cdots \\
\cdots\end{array}$ & $\begin{array}{l}\text { Br. } \\
\text { Br. } \\
\text { See } \\
\text { See }\end{array}$ & $\begin{array}{l}\text { Clearly divided. } \\
\text { Clearly divided. }\end{array}$ \\
\hline $\begin{array}{l}1897.618 \\
1899.564\end{array}$ & & & & $\begin{array}{l}\text { I20. } 4 \\
116.6\end{array}$ & $\begin{array}{l}0.62 \\
\text { o. } 87\end{array}$ & & & & & & \\
\hline I $899 \cdot 40 \mathrm{I}$ & $\Sigma$ 1969.. & 153924 & $+60 \times 8$ & $54 \cdot 3$ & 0.63 & 5,6 & I & 26-inch & 600 & $\mathrm{Br}$. & \\
\hline $\begin{array}{r}1903.514 \\
.519 \\
\end{array}$ & $\Sigma_{1974 \ldots}$ & 154359 & $|-256|$ & $\begin{array}{l}162.9 \\
\mathrm{I} 61.7 \\
\end{array}$ & $\begin{array}{l}2.56 \\
2.52 \\
\end{array}$ & $\begin{array}{l}4,6 \\
4,6\end{array}$ & $\begin{array}{l}4 \\
4\end{array}$ & "26-inch & $\begin{array}{l}600 \\
600\end{array}$ & $\begin{array}{l}\text { Sk. } \\
\text { Sk. }\end{array}$ & $\begin{array}{l}\text { Mags. 9.0-9.o. } \\
\text { Mags. 9.0-9.o. }\end{array}$ \\
\hline $1903 \cdot 516$ & & & & 162.3 & 2.54 & & & & & & \\
\hline $\mathbf{1 8 9 5 . 5 4 9}$ & Skinner $9 \ldots$ & 154641 & $-16 \quad 56$ & $279 \cdot 5$ & 2. 19 & 4,4 & .. & 26-inch & 400 & F. & $\begin{array}{l}\text { Mags., about } \\
\text { equa } 1, \quad 9.0 ; \\
\text { seeing poor. }\end{array}$ \\
\hline $\begin{array}{r}1900.357 \\
.475\end{array}$ & & & & $\begin{array}{l}278.9 \\
281.0\end{array}$ & $\begin{array}{l}2.34 \\
2.6 \mathrm{I}\end{array}$ & $\begin{array}{l}4,8 \\
4,8\end{array}$ & $\begin{array}{l}\mathrm{g} \\
. .\end{array}$ & 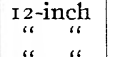 & $\cdots$ & B. & \\
\hline .573 & & & & 278.0 & 2.01 & 4,8 & .. & “" “ & $\cdots$ & B. & $\begin{aligned} \text { M a gs. } & 9.6-9.8 \\
\text { very } & \text { difficult; }\end{aligned}$ \\
\hline $\begin{array}{l}\mathrm{r} 895.549 \\
\mathrm{rg00.468}\end{array}$ & & & & $\begin{array}{l}279 \cdot 5 \\
279.3\end{array}$ & $\begin{array}{l}\text { 2. } 19 \\
2.32\end{array}$ & & & & & & faint. \\
\hline $\begin{array}{r}1899.399 \\
.423 \\
\end{array}$ & $\beta 810$. & I5 4736 & +4246 & $\begin{array}{l}82.9 \\
87.8 \\
\end{array}$ & $\begin{array}{l}0.96 \\
0.88 \\
\end{array}$ & $\begin{array}{l}4,4 \\
4,4\end{array}$ & $\begin{array}{l}2 \\
3\end{array}$ & 26-inch & $\begin{array}{l}600 \\
600\end{array}$ & $\begin{array}{l}\mathrm{Br} . \\
\mathrm{Br} .\end{array}$ & $\begin{array}{l}\text { Mags. } 8.5 \text { - Ir.o; } \\
\text { very difficult. }\end{array}$ \\
\hline $\mathrm{I} 899.4 \mathrm{II}$ & & & & $85 \cdot 4$ & 0.92 & & & & & & - \\
\hline $\begin{array}{r}1899 \cdot 582 \\
1900.584 \\
.590 \\
1903 \cdot 519 \\
\end{array}$ & $\Sigma 1985 \ldots$ & I 55044 & - I 52 & $\begin{array}{l}336.5 \\
338.3 \\
335.3 \\
335.8 \\
\end{array}$ & $\begin{array}{l}6.02 \\
5.49 \\
6.02 \\
6.13 \\
\end{array}$ & $\begin{array}{l}8,16 \\
4,8 \\
4,8 \\
4,6\end{array}$ & $\begin{array}{l}5 \\
\cdots \\
\ddot{3}\end{array}$ & $\begin{array}{l}\text { I2-inch } \\
\text { "." " } \\
26 \text {-inch }\end{array}$ & $\begin{array}{l}\cdots \\
\cdots \\
600\end{array}$ & $\begin{array}{l}\text { See } \\
\text { B. } \\
\text { B. } \\
\text { Sk. }\end{array}$ & $\begin{array}{l}\text { H્ II. } 85 . \\
\text { Mags. 6.0-5.5. } \\
\text { Mags. 7.0-9.5. }\end{array}$ \\
\hline $\begin{array}{l}1899.582 \\
1900.587 \\
1903.519\end{array}$ & & & & $\begin{array}{l}336.5 \\
336.8 \\
335.8\end{array}$ & $\begin{array}{l}6.02 \\
5.76 \\
6.13\end{array}$ & & & & & & \\
\hline 1903.519 & $\Sigma$ 3101.. & I $5534 \mathrm{I}$ & -247 & 61.3 & 2.53 & 4,6 & 3 & 26 -inch & 600 & Sk. & Mags. 9.0-9.o. \\
\hline $\begin{array}{r}1903.5^{14} \\
.517 \\
\end{array}$ & $\beta 623 \ldots \ldots$ & I5 5555 & $-64 \mathrm{I}$ & $\begin{array}{r}232.8 \\
233.2 \\
\end{array}$ & $\begin{array}{l}\text { I. OI } \\
\text { O. } 97 \\
\end{array}$ & $\begin{array}{l}4,6 \\
4,6\end{array}$ & $\begin{array}{c}3-4 \\
3\end{array}$ & 26-inch & $\begin{array}{l}600 \\
600\end{array}$ & $\begin{array}{l}\text { Sk. } \\
\text { Sk. }\end{array}$ & $\begin{array}{l}\text { Mags. } 9 \cdot 5^{-9}-5 . \\
\text { Mags. } 9 \cdot 3-9 \cdot 3 \text {. }\end{array}$ \\
\hline 1903.516 & & & & 233.0 & 0.99 & & . & & & & \\
\hline
\end{tabular}




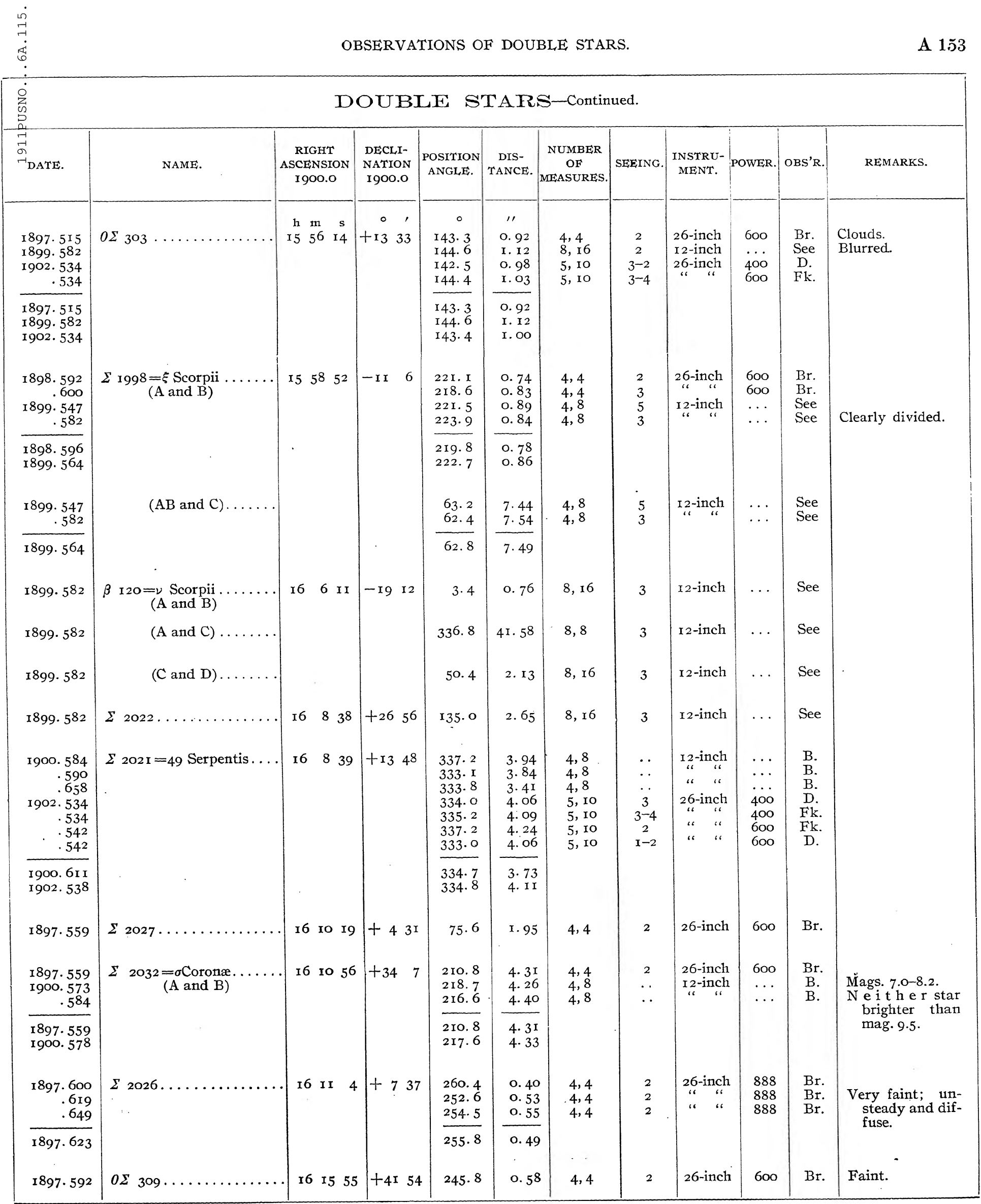




\begin{tabular}{|c|c|c|c|c|c|c|c|c|c|c|c|}
\hline \multicolumn{12}{|c|}{ DOUBLE STARS-Continued. } \\
\hline DATE. & NAME. & $\begin{array}{l}\text { RIGHT } \\
\text { ASCENSION } \\
\text { I } 900.0\end{array}$ & $\begin{array}{l}\text { DECLI- } \\
\text { NATION } \\
\text { I9OO.O }\end{array}$ & $\begin{array}{l}\text { POSITION } \\
\text { ANGLE. }\end{array}$ & $\begin{array}{c}\text { DIS- } \\
\text { TANCE. }\end{array}$ & $\mid \begin{array}{c}\text { NUMBER } \\
\text { OF } \\
\text { MEASURES. }\end{array}$ & SEEING. & $\begin{array}{c}\text { INSTRU- } \\
\text { MENT. }\end{array}$ & POWER. & OBS'R. & REMARKS. \\
\hline 1897.575 & $\Sigma 2041 \ldots \ldots \ldots \ldots \ldots$ & $\begin{array}{rrr}11 & n & s \\
\text { I } & 16 & 43\end{array}$ & $\begin{array}{r}0 \\
+\quad 127\end{array}$ & $\begin{array}{c}\circ \\
359.0\end{array}$ & 2.68 & 4,4 & 2 & 26 -inch & 600 & Br. & \\
\hline $\begin{array}{r}1900.595 \\
.612 \\
\end{array}$ & $\Sigma 2044 \ldots \ldots \ldots \ldots \ldots$ & 162036 & +3716 & $\begin{array}{l}342.6 \\
344.0\end{array}$ & $\begin{array}{l}8.60 \\
8.93\end{array}$ & $\begin{array}{l}4,8 \\
4,8\end{array}$ & $\begin{array}{l}\cdots \\
\cdots\end{array}$ & I 2 -inch & $\begin{array}{l}\cdots \\
\cdots\end{array}$ & $\begin{array}{l}\text { B. } \\
\text { B. }\end{array}$ & \\
\hline 1900.604 & & & & $343 \cdot 3$ & 8.76 & & & & & & \\
\hline $\begin{array}{r}1900.584 \\
.590 \\
\end{array}$ & $\Sigma 2054=99$ Draconis.... & $1622 \quad 28$ & +6155 & $\begin{array}{l}356.5 \\
360.2\end{array}$ & $\begin{array}{l}\text { 1. } 32 \\
1.28\end{array}$ & $\begin{array}{l}4,8 \\
4,8\end{array}$ & $\cdots$ & 1 2 -inch & $\begin{array}{l}\cdots \\
\cdots\end{array}$ & $\begin{array}{l}\text { B. } \\
\text { B. }\end{array}$ & \\
\hline I900. 587 & & & & 358.4 & I. 30 & & & & & & \\
\hline $\begin{array}{r}1899.544 \\
1900.595 \\
.612 \\
1902.525 \\
.525 \\
\end{array}$ & $\alpha$ Scorpii............. & 162316 & $\begin{array}{ll}-26 & 13\end{array}$ & $\begin{array}{l}276.1 \\
265.5 \\
268.3 \\
273 \cdot 3 \\
275.5\end{array}$ & $\begin{array}{l}3 \cdot 5 \mathrm{I} \\
3 \cdot 16 \\
3 \cdot 43 \\
3 \cdot 22 \\
3 \cdot 04\end{array}$ & $\begin{array}{l}4,6 \\
4,8 \\
4,8 \\
5,10 \\
5,10\end{array}$ & $\begin{array}{l}3 \\
\cdots \\
\because \\
4 \\
4\end{array}$ & $\begin{array}{c}\text { 12-inch } \\
\text { “" } " ، \\
\text { 26-inch } \\
\text { ". }\end{array}$ & $\begin{array}{l}\cdots \\
\cdots \\
600 \\
600\end{array}$ & $\begin{array}{l}\text { See } \\
\text { B. } \\
\text { B. } \\
\text { Fk. } \\
\text { D. }\end{array}$ & Cloudy. \\
\hline $\begin{array}{l}1899.544 \\
1900.604 \\
1902.525\end{array}$ & & & & $\begin{array}{l}276.1 \\
266.9 \\
274.4\end{array}$ & $\begin{array}{l}3.5 \mathrm{I} \\
3 \cdot 30 \\
3.13\end{array}$ & & & & & & \\
\hline 1903.563 & $\Sigma 2048 \ldots \ldots$ & 162325 & -754 & 299.8 & $5 \cdot 15$ & 4,6 & $3-4$ & 26-inch & 600 & Sk. & Mags. 7.0-ro.o. \\
\hline I 896.567 & $\Sigma 2049 \ldots \ldots \ldots$ & 162348 & $+26 \quad 12$ & 208.7 & 1.23 & 3,4 & 2 & 26-inch & 600 & $\mathrm{Br}$. & \\
\hline $\begin{array}{r}1897.559 \\
.575 \\
1900.595 \\
.612 \\
\end{array}$ & $\Sigma 2052=71$ Herculis. & 162430 & tr8 37 & $\begin{array}{l}95 \cdot 3 \\
92 \cdot 4 \\
95 \cdot 9 \\
97 \cdot 1\end{array}$ & $\begin{array}{l}1.83 \\
2.01 \\
2.07 \\
2.19\end{array}$ & $\begin{array}{l}4,4 \\
4,4 \\
4,8 \\
4,8\end{array}$ & $\begin{array}{l}\mathbf{I} \\
2 \\
\cdots \\
\cdots\end{array}$ & $\begin{array}{l}26-\text { inch } \\
\text { 1 } \\
\text { 12-inch }\end{array}$ & $\begin{array}{l}600 \\
600 \\
\cdots \\
\cdots\end{array}$ & $\begin{array}{l}\mathrm{Br} . \\
\mathrm{Br} . \\
\mathrm{B} . \\
\mathrm{B} .\end{array}$ & \\
\hline $\begin{array}{l}1897.567 \\
1900.604\end{array}$ & & & & $\begin{array}{l}93.8 \\
96.5\end{array}$ & $\begin{array}{l}\text { I. } 92 \\
2.13\end{array}$ & & & & & & \\
\hline 1907.517 & $\Sigma 3104 \ldots \ldots \ldots \ldots \ldots$ & $162+53$ & -1420 & $47 \cdot 8$ & $9 \cdot 5^{2}$ & 4,6 & $f-g$ & 26-inch & 400 & $\mathrm{R}$. & Mags. 8.8-9.2. \\
\hline $\begin{array}{r}1896.567 \\
1899.544 \\
1900.595 \\
.612 \\
.699 \\
\end{array}$ & $\Sigma 2055=\lambda$ Ophiuchi & 162552 & $1+212$ & $\begin{array}{l}49 \cdot 9 \\
55 \cdot 5 \\
47 \cdot 3 \\
60.2 \\
53.8\end{array}$ & $\begin{array}{l}\text { I. } 47 \\
\text { I. } 78 \\
\text { I. } 60 \\
\text { I. } 92 \\
\text { I. } 58\end{array}$ & $\begin{array}{l}3,4 \\
4,6 \\
4,8 \\
4,8 \\
4,8\end{array}$ & $\begin{array}{l}2 \\
3 \\
\cdots \\
\cdots \\
\cdots\end{array}$ & 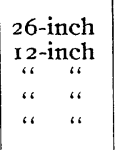 & $\begin{array}{l}600 \\
\cdots \\
\cdots \\
\cdots\end{array}$ & $\begin{array}{l}\text { Br. } \\
\text { See } \\
\text { B. } \\
\text { B. } \\
\text { B. }\end{array}$ & $\begin{array}{l}\text { Unsteady. } \\
\text { Ve r y difficult; } \\
\text { indistinct. }\end{array}$ \\
\hline $\begin{array}{l}1896.567 \\
1899.544 \\
1900.635\end{array}$ & & & & $\begin{array}{l}49.9 \\
55 \cdot 5 \\
53.8\end{array}$ & $\begin{array}{l}\text { I. } 47 \\
\text { I. } 78 \\
\text { I. } 70\end{array}$ & & & & & & \\
\hline $1897 \cdot 592$ & $O \Sigma_{313} \ldots \ldots$ & 162910 & +4020 & I5O. 0 & $0.9 \mathrm{r}$ & 4,4 & 3 & 26-inch & 600 & $\mathrm{Br}$. & \\
\hline $1897 \cdot 655$ & $0 \Sigma 314 \ldots$ & 163431 & +2040 & 236.9 & 3.59 & 4,4 & 2 & 26-inch & 400 & $\mathrm{Br}$. & Bad. \\
\hline $\begin{array}{r}\text { I } 899.859 \\
\quad .861 \\
\end{array}$ & $\Sigma 2084=\zeta$ Herculis $\ldots$ & 163732 & $+3 \times 47$ & $\begin{array}{r}25^{8.3} \\
27^{2} .3 \\
\end{array}$ & $\begin{array}{l}\text { o. } 33 \\
\text { o. } 37 \\
\end{array}$ & $\begin{array}{l}3,4 \\
3,4\end{array}$ & $\begin{array}{l}\cdots \\
\cdots\end{array}$ & 26-inch & $\cdots$ & $\begin{array}{l}\text { See } \\
\text { See }\end{array}$ & $\begin{array}{l}\text { Not separated. } \\
\text { Unsteady; not } \\
\text { separated. }\end{array}$ \\
\hline 1899.860 & & & & $265 \cdot 3$ & o. 35 & & & & & & \\
\hline
\end{tabular}




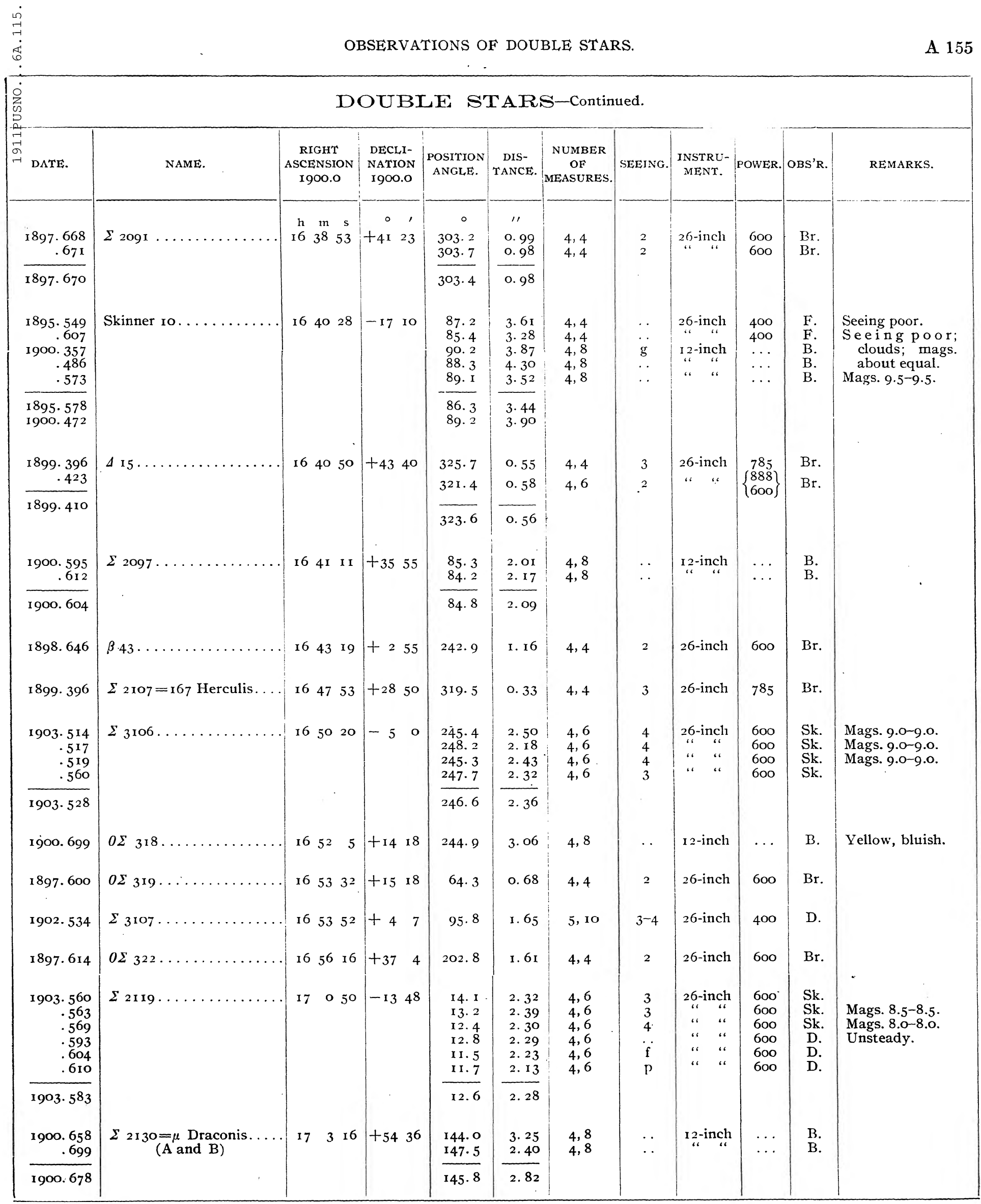




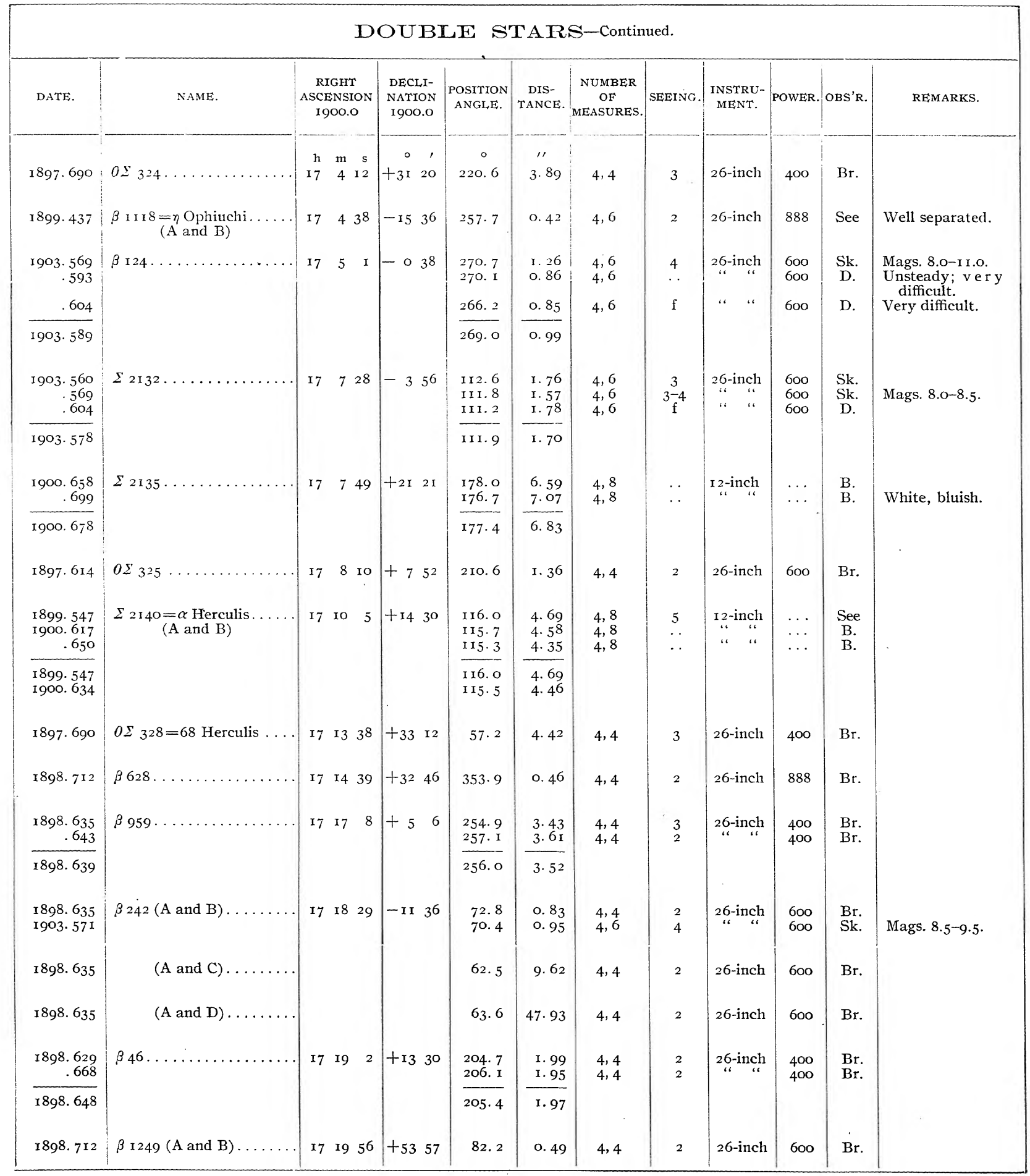




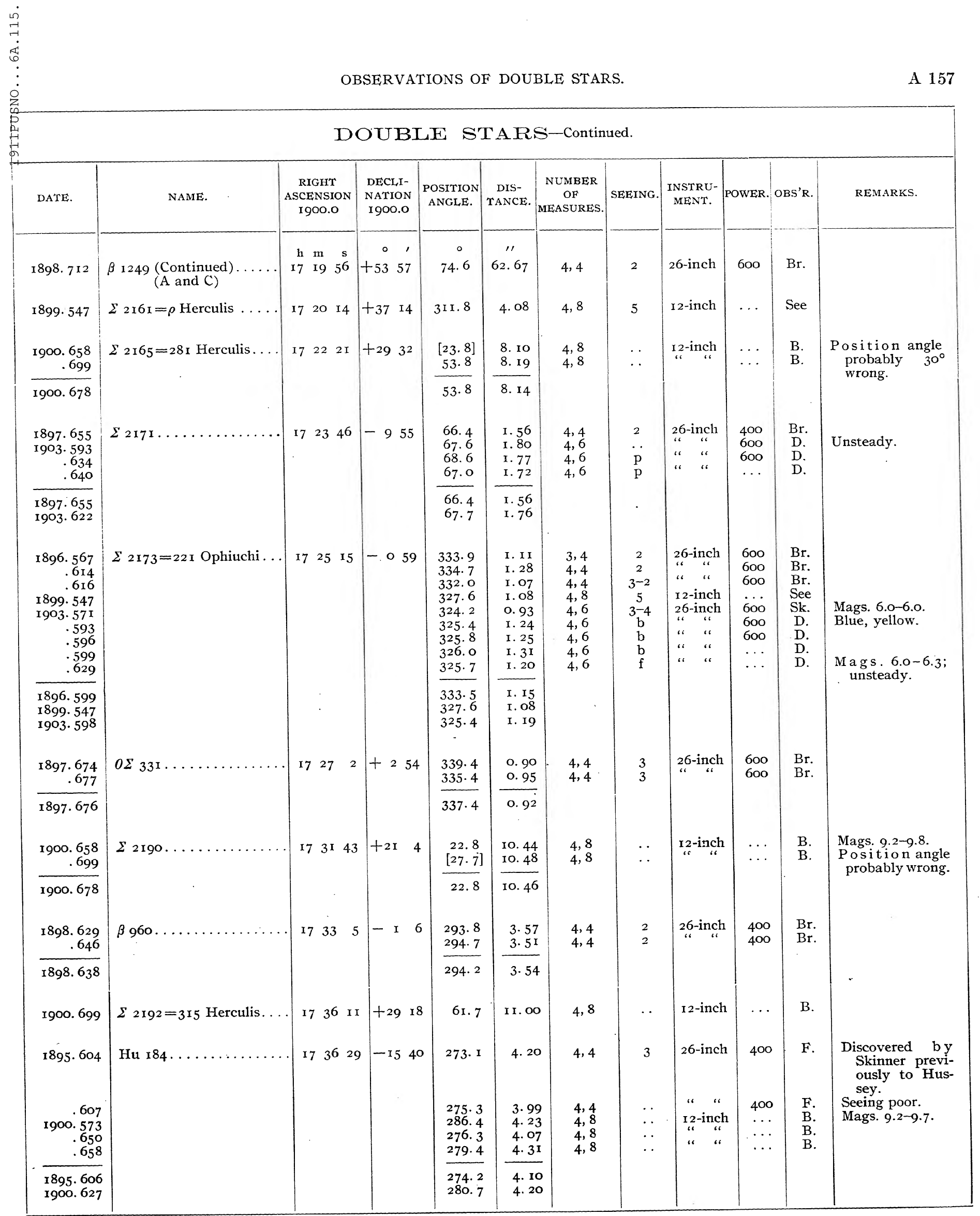




\begin{tabular}{|c|c|c|c|c|c|c|c|c|c|c|c|}
\hline \multicolumn{12}{|c|}{ DOUBLE STARS-Continued. } \\
\hline DATE. & NAME. & $\begin{array}{c}\text { RIGHT } \\
\text { ASCENSION } \\
\text { I } 900.0\end{array}$ & $\begin{array}{l}\text { DECLI- } \\
\text { NATION } \\
\text { I9O0.0 }\end{array}$ & $\begin{array}{c}\text { POSITION } \\
\text { ANGLE. }\end{array}$ & $\begin{array}{c}\text { DIS- } \\
\text { TANCE. }\end{array}$ & $\begin{array}{c}\text { NUMBER } \\
\text { OF } \\
\text { MEASURES. }\end{array}$ & SEEING. & $\begin{array}{l}\text { INSTRU- } \\
\text { MENT. }\end{array}$ & POWER. & OBS'R. & REMARKS. \\
\hline 1898.676 & $\beta 1251 \ldots \ldots \ldots \ldots \ldots$ & \begin{tabular}{ccc|}
$\mathrm{h}$ & $\mathrm{m}$ & $\mathrm{s}$ \\
17 & 37 & 29
\end{tabular} & 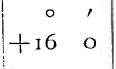 & $\begin{array}{c}\circ \\
69.2\end{array}$ & 1. 40 & 4,4 & 2 & 26-inch & 600 & Br. & \\
\hline $\begin{array}{r}1900.696 \\
.699 \\
\end{array}$ & $\Sigma 2218 \ldots \ldots \ldots \ldots \ldots$ & 173940 & +6343 & $\begin{array}{l}338.7 \\
338.5 \\
\end{array}$ & $\begin{array}{l}2.01 \\
2.24 \\
\end{array}$ & $\begin{array}{l}4,8 \\
4,8\end{array}$ & $\begin{array}{l}\cdots \\
\cdots\end{array}$ & 1 2 -inch & $\begin{array}{l}\cdots \\
\cdots\end{array}$ & $\begin{array}{l}\text { B. } \\
\text { B. }\end{array}$ & . \\
\hline 1900.698 & & & & 338.6 & 2.12 & & & & & & \\
\hline $\begin{array}{r}1900.573 \\
.595 \\
\end{array}$ & $\Sigma 2205 \ldots \ldots \ldots$ & $1741 \quad 16$ & +1745 & $\begin{array}{r}309 \cdot 4 \\
308 \cdot 5 \\
\end{array}$ & $\begin{array}{l}1.78 \\
2.57 \\
\end{array}$ & $\begin{array}{l}4,8 \\
4,8\end{array}$ & $\cdots$ & I 2 -inch & $\cdots$ & $\begin{array}{l}\text { B. } \\
\text { B. }\end{array}$ & \\
\hline I900. $5^{84}$ & & & & 309.0 & 2. 18 & & & & & & \\
\hline $\begin{array}{r}1897.619 \\
.649 \\
.655 \\
1906.422 \\
.482 \\
.482 \\
.668 \\
.668 \\
\end{array}$ & $\begin{array}{c}\text { A. Clark } 7=\mu \text { Herculis... } \\
(\mathrm{B} \text { and } \mathrm{C})\end{array}$ & I7 4233 & +2747 & $\begin{array}{l}50.0 \\
52.6 \\
52.7 \\
74.0 \\
72.1 \\
74.2 \\
73.1 \\
71.6 \\
\end{array}$ & $\begin{array}{l}1.42 \\
1.51 \\
\text { I. } 50 \\
\text { I. } 54 \\
1.45 \\
\text { I. } 40 \\
\text { I. } 36 \\
\text { I. } 69 \\
\end{array}$ & $\begin{array}{l}4,4 \\
4,4 \\
4,4 \\
4,6 \\
4,6 \\
3,6 \\
4,6 \\
3,6\end{array}$ & $\begin{array}{c}2 \\
I-2 \\
I-2 \\
f \\
g \\
g \\
f \\
p-f\end{array}$ & 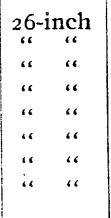 & $\begin{array}{l}400 \\
600 \\
400 \\
400 \\
785 \\
785 \\
600 \\
600\end{array}$ & $\begin{array}{l}\text { Br. } \\
\text { Br. } \\
\text { Br. } \\
\text { Hd. } \\
\text { Hd. } \\
\text { Fr. } \\
\text { Hd. } \\
\text { Fr. }\end{array}$ & $\begin{array}{l}\text { A and } B=\Sigma \\
2220 . \\
\text { Bright field. }\end{array}$ \\
\hline $\begin{array}{l}\text { I } 897.641 \\
1906.544\end{array}$ & & & & $\begin{array}{l}5 \mathrm{I} .8 \\
73.0\end{array}$ & $\begin{array}{l}\text { I. } 48 \\
\text { I. } 49\end{array}$ & & & & & & \\
\hline $\begin{array}{l}1898.670 \\
1903.629\end{array}$ & $\beta 824 \ldots \ldots \ldots \ldots \ldots$ & I7 4342 & - I 50 & $\begin{array}{l}35 \mathrm{r} \cdot 3 \\
35 \mathrm{r} \cdot 4\end{array}$ & $\begin{array}{l}0.63 \\
0.72\end{array}$ & $\begin{array}{l}4,4 \\
4,6\end{array}$ & f & 26 -inch & $\begin{array}{l}888 \\
\cdots\end{array}$ & $\begin{array}{l}\mathrm{Br} \\
\mathrm{D} .\end{array}$ & \\
\hline I898. 597 & $0 \Sigma 338 \ldots \ldots$ & 17 4728 & +1521 & 19.0 & 0.66 & 4,8 & 3 & 26-inch & $\left\{\begin{array}{l}600 \\
888\end{array}\right\}$ & Br. & \\
\hline $\begin{array}{l}1899 \cdot 328 \\
1897.616\end{array}$ & $\begin{array}{l}\text { A. Clark } 9 \ldots \\
\Sigma 2244 \ldots \ldots\end{array}$ & $\begin{array}{lll}17 & 50 & 17 \\
17 & 51 & 57\end{array}$ & $\begin{array}{l}+2950 \\
+05\end{array}$ & $\begin{array}{l}148.8 \\
275.6\end{array}$ & $\begin{array}{l}\text { I. } 38 \\
\text { I. } 12\end{array}$ & $\begin{array}{l}4,4 \\
4,4\end{array}$ & $\begin{array}{c}2 \\
2-3\end{array}$ & $\begin{array}{l}\text { 26-inch } \\
\text { 26-inch }\end{array}$ & $\begin{array}{l}888 \\
600\end{array}$ & $\begin{array}{l}\mathrm{Br} . \\
\mathrm{Br} .\end{array}$ & $\begin{array}{l}\text { Position angle } \\
\text { not in agree- } \\
\text { ment with } \\
\text { other observ- } \\
\text { ers. }\end{array}$ \\
\hline $\begin{array}{r}1898.643 \\
.670 \\
\end{array}$ & $\beta 417 \ldots$ & I 75254 & +3927 & $\begin{array}{r}274.3 \\
272.0 \\
\end{array}$ & $\begin{array}{l}\text { I. } 52 \\
\text { I. } 57 \\
\end{array}$ & $\begin{array}{l}4,4 \\
4,4\end{array}$ & $\begin{array}{l}3 \\
2\end{array}$ & 26-inch & $\begin{array}{l}400 \\
600\end{array}$ & $\begin{array}{l}\mathrm{Br} . \\
\mathrm{Br}\end{array}$ & \\
\hline 1898.656 & & & & 273.2 & I. 54 & & & & & & \\
\hline $\begin{array}{r}1896.598 \\
.601 \\
.616 \\
1899.547 \\
1900.617 \\
.650 \\
1903.593 \\
.596 \\
.599 \\
.604 \\
.610 \\
\end{array}$ & $\Sigma 2262=\tau$ Ophiuchi.... & I7 $57 \quad 38$ & $-8 \mathrm{II}$ & $\begin{array}{l}255.8 \\
256.1 \\
258.6 \\
255 \cdot 4 \\
259.1 \\
259.6 \\
260.0 \\
259.9 \\
259.3 \\
261.1 \\
260.4 \\
\end{array}$ & $\begin{array}{l}1.99 \\
1.96 \\
1.93 \\
2.24 \\
2.06 \\
2.08 \\
1.97 \\
2.06 \\
2.09 \\
2.07 \\
2.01 \\
\end{array}$ & $\begin{array}{l}3,4 \\
3,4 \\
4,4 \\
4,8 \\
4,8 \\
4,8 \\
4,6 \\
4,6 \\
4,6 \\
4,6 \\
4,6\end{array}$ & $\begin{array}{c}\cdots \\
3 \\
\mathrm{I}-2 \\
5 \\
\cdots \\
\cdots \\
\mathrm{b} \\
\mathrm{b} \\
\mathrm{b} \\
\mathrm{f} \\
\mathrm{p}\end{array}$ & 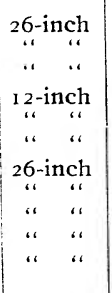 & $\begin{array}{l}400 \\
600 \\
600 \\
\ldots \\
\ldots \\
\ldots \\
600 \\
600 \\
\ldots \\
400 \\
600\end{array}$ & $\begin{array}{l}\mathrm{Br} . \\
\mathrm{Br} . \\
\mathrm{Br} . \\
\text { See } \\
\text { B. } \\
\text { B. } \\
\text { D. } \\
\text { D. } \\
\text { D. } \\
\text { D. } \\
\text { D. }\end{array}$ & Unsteady. \\
\hline $\begin{array}{l}1896.605 \\
\text { I } 899.547 \\
1900.634 \\
1903.600\end{array}$ & & & & $\begin{array}{l}256.8 \\
255.4 \\
259.4 \\
260.1\end{array}$ & $\begin{array}{l}1.96 \\
2.24 \\
2.07 \\
2.04\end{array}$ & & & . & & & . \\
\hline
\end{tabular}




\begin{tabular}{|c|c|c|c|c|c|c|c|c|c|c|c|}
\hline \multicolumn{12}{|l|}{$\begin{array}{l}\dot{0} \\
Z_{2}\end{array}$} \\
\hline $\begin{array}{l}\text { ने } \\
\text { DATE. }\end{array}$ & NAME. & $\begin{array}{c}\text { RIGHT } \\
\text { ASCENSION } \\
\text { IgOO.O }\end{array}$ & $\begin{array}{l}\text { DECLI- } \\
\text { NATION } \\
\text { I } 900.0\end{array}$ & $\begin{array}{c}\text { POSITION } \\
\text { ANGLE. }\end{array}$ & $\begin{array}{c}\text { DIS- } \\
\text { TANCE. }\end{array}$ & $\begin{array}{c}\text { NUMBER } \\
\text { OF } \\
\text { MEASURES. }\end{array}$ & SEEING. & $\begin{array}{l}\text { INSTRU- } \\
\text { MENT. }\end{array}$ & POWER. & OBS'R. & REMARKS. \\
\hline $\begin{array}{r}\mathrm{r} 898.629 \\
.668 \\
\end{array}$ & $\beta 635(\mathrm{~A}$ and $\mathrm{B}) \ldots \ldots$ & $\begin{array}{llc}\mathrm{h} & \mathrm{m} & \mathrm{s} \\
\mathrm{I} 7 & 57 & 42\end{array}$ & $\begin{array}{r}0 \\
+\quad 137\end{array}$ & $\begin{array}{l}\text { I } 6.6 \\
\text { I } 6.8\end{array}$ & $\begin{array}{l}\text { ' } \\
\text { I. } 45 \\
\text { I. } 53\end{array}$ & $\begin{array}{l}5,4 \\
4,6\end{array}$ & $\begin{array}{l}2 \\
2\end{array}$ & 26-inch & $\begin{array}{l}600 \\
600\end{array}$ & $\begin{array}{l}\mathrm{Br} . \\
\mathrm{Br} .\end{array}$ & \\
\hline 1898.648 & & & & II 6.7 & I. 49 & & & & & 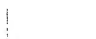 & \\
\hline $\begin{array}{r}1898.629 \\
.668\end{array}$ & $(A$ and $C) \ldots \ldots$. & & & $\begin{array}{l}\text { I } 22.0 \\
\text { I } 22.1\end{array}$ & $\begin{array}{l}69 \cdot 27 \\
69 \cdot 16\end{array}$ & $\begin{array}{l}4,4 \\
4,4\end{array}$ & $\begin{array}{l}2 \\
2\end{array}$ & 26-inch & $\begin{array}{l}600 \\
600\end{array}$ & $\begin{array}{l}\mathrm{Br} . \\
\mathrm{Br} \text {. }\end{array}$ & \\
\hline I 898.648 & & & & 122.0 & 69.22 & & & & & & \\
\hline I900. 699 & $\Sigma 2271 \ldots \ldots \ldots \ldots \ldots$ & $175^{8} 8$ & $+5^{2} 5^{x}$ & $267 \cdot 9$ & 2.42 & 4,8 & $\cdots$ & I 2-inch & $\cdots$ & B. & \\
\hline I900. 699 & $\Sigma 2267 \ldots \ldots \ldots \ldots \ldots$ & I7 $5^{8} 27$ & $+40 \cdot 11$ & 246.2 & I. 28 & 4,8 & $\ldots$ & I 2 -inch & $\ldots$ & B. & \\
\hline I 897.649 & $\Sigma 2275 \ldots \ldots \ldots \ldots \ldots$ & I8 00 & +3921 & I 35.8 & 0.75 & 4,4 & 3 & 26-inch & 600 & $\mathrm{Br}$ & \\
\hline $\begin{array}{r}\mathrm{I} 896.598 \\
.601 \\
.671 \\
\mathrm{I} 898.720 \\
.722 \\
\mathrm{I} 899.412 \\
.421 \\
.426 \\
.429 \\
.434 \\
.544 \\
.547 \\
.703 \\
\mathrm{I} 900.535 \\
.538 \\
.540 \\
.595 \\
.595 \\
.612 \\
.617 \\
.628 \\
.650 \\
.658 \\
\mathrm{I} 901.490 \\
.586 \\
\mathrm{I} 902.632\end{array}$ & $\begin{array}{c}\Sigma 2272=70 \text { Ophiuchi } \ldots . \\
(\mathrm{A} \text { and } \mathrm{B})\end{array}$ & I 8 o 24 & $+23 I$ & $\begin{array}{l}293 \cdot 1 \\
290.3 \\
281.2 \\
271 \cdot 3 \\
271.8 \\
263.2 \\
262.4 \\
261.4 \\
260.2 \\
261.4 \\
261.4 \\
260.3 \\
263 \cdot 6 \\
248.1 \\
250.5 \\
245 \cdot 6 \\
247 \cdot 6 \\
247.8 \\
256.4 \\
244.0 \\
242.2 \\
247 \cdot 9 \\
\\
244 \cdot 7 \\
232.7 \\
231.2 \\
210.3\end{array}$ & $\begin{array}{l}2.33 \\
2.29 \\
2.07 \\
2.00 \\
1.86 \\
1.77 \\
1.80 \\
1.79 \\
1.98 \\
1.97 \\
2.08 \\
1.79 \\
2.07 \\
1.91 \\
1.81 \\
1.91 \\
1.72 \\
1.85 \\
\text { I. } 88 \\
1.92 \\
1.90 \\
1.67 \\
1.69 \\
\text { I. } 88 \\
\text { I. } 90 \\
\text { I. } 89\end{array}$ & $\begin{array}{l}3,4 \\
3,4 \\
4,4 \\
4,4 \\
4,4 \\
8,8 \\
4,6 \\
4,4 \\
4,6 \\
4,6 \\
4,8 \\
4,8 \\
4,8 \\
5,8 \\
5,8 \\
9,10 \\
5,8 \\
4,8 \\
4,8 \\
4,8 \\
4,8 \\
4,8 \\
\\
4,8 \\
8,15 \\
4,8 \\
5,10\end{array}$ & $\begin{array}{c}3 \\
3 \\
3 \\
2 \\
4 \\
2 \\
3 \\
2 \\
3 \\
\cdots \\
3 \\
5 \\
\ldots \\
4-5 \\
4 \\
3-4 \\
2 \\
\ldots \\
\ldots \\
\ldots \\
4 \\
\ldots \\
\ldots \\
3 \\
3 \\
3\end{array}$ & 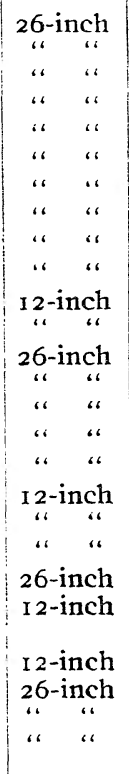 & 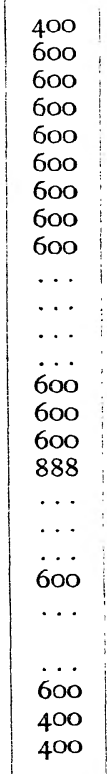 & \begin{tabular}{|c} 
Br. \\
Br. \\
Br. \\
Br. \\
Br. \\
Br. \\
Br. \\
Br. \\
See \\
See \\
See \\
See \\
See \\
See \\
See \\
See \\
See \\
B. \\
B. \\
B. \\
See \\
B. \\
B. \\
B. \\
See \\
See \\
See
\end{tabular} & $\begin{array}{l}\text { Unsteady; blaz- } \\
\text { ing. } \\
\text { Star blazing ; } \\
\text { poor. } \\
\\
\text { Better than for- } \\
\text { mer measures. } \\
\text { Twilight; no illu- } \\
\text { mination. } \\
\text { Blurred and un- } \\
\text { steady. }\end{array}$ \\
\hline $\begin{array}{l}1896.623 \\
1898.721 \\
1899.490 \\
\text { 1900. } 597 \\
\text { 1901. } 538 \\
1902.632\end{array}$ & . & & & $\begin{array}{l}288.2 \\
271.6 \\
261.7 \\
247.5 \\
232.0 \\
210.3\end{array}$ & $\begin{array}{l}\text { 2. } 23 \\
\text { I. } 93 \\
\text { I. } 9 \text { I } \\
\text { I. } 83 \\
\text { I. } 89 \\
\text { I. } 89\end{array}$ & & & & & & $*$ \\
\hline $\begin{array}{r}1899.429 \\
.434 \\
1900.535 \\
.538 \\
.540 \\
.628 \\
\end{array}$ & $(B$ and $C) \ldots \ldots$ & & & $\begin{array}{l}207.4 \\
209.0 \\
209.9 \\
210.4 \\
209.8 \\
209.7\end{array}$ & $\begin{array}{l}50.83 \\
50.77 \\
49.51 \\
50.28 \\
49.79 \\
49.30\end{array}$ & $\begin{array}{l}4,8 \\
4,8 \\
4,8 \\
4,8 \\
5,10 \\
4,8\end{array}$ & $\begin{array}{c}3 \\
\cdots \\
4-5 \\
4 \\
3-4 \\
4\end{array}$ & 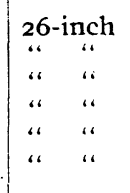 & $\begin{array}{l}600 \\
6 \\
600 \\
600 \\
600 \\
600\end{array}$ & $\begin{array}{l}\text { See } \\
\text { See } \\
\text { See } \\
\text { See } \\
\text { See } \\
\text { See }\end{array}$ & $\begin{array}{l}\text { Measures with } \\
\text { great care. }\end{array}$ \\
\hline $\begin{array}{l}\text { I } 899.432 \\
\text { 1900. } 560\end{array}$ & & & & $\begin{array}{l}208.2 \\
209.9\end{array}$ & $\begin{array}{l}50.80 \\
49.72\end{array}$ & & & & & & \\
\hline
\end{tabular}




\begin{tabular}{|c|c|c|c|c|c|c|c|c|c|c|c|c|}
\hline \multicolumn{13}{|c|}{ DOUBLE STARS-Continued. } \\
\hline DATE. & NAME. & $\begin{array}{r}\text { RI } \\
\text { ASCE } \\
19\end{array}$ & $\begin{array}{l}\text { GHT } \\
\text { ENSION } \\
\text { OOO.O }\end{array}$ & $\begin{array}{l}\text { DECLI- } \\
\text { NATION } \\
\text { I9OO.0 }\end{array}$ & $\begin{array}{c}\text { POSITION } \\
\text { ANGLE. }\end{array}$ & \begin{tabular}{|c|} 
DIS- \\
TANCE.
\end{tabular} & $\begin{array}{c}\text { NUMBER } \\
\text { OF } \\
\text { MEASURES. }\end{array}$ & SEEING. & $\begin{array}{l}\text { INSTRU- } \\
\text { MENT. }\end{array}$ & POWER. & OBS'R. & REMARKS. \\
\hline 1899.426 & $\underset{(A \text { and } \mathrm{C})}{\Sigma 2272-(\text { Continued }) \ldots}$ & $\begin{array}{r}\mathrm{h} \\
\mathrm{I} 8\end{array}$ & $\begin{array}{lr}m & s \\
0 & 24\end{array}$ & $\begin{array}{r}0 \\
+231\end{array}$ & $\begin{array}{c}\circ \\
209 \cdot 7\end{array}$ & $\begin{array}{c}\prime \prime \\
51.97\end{array}$ & 4,4 & 2 & 26-inch & 600 & $\mathrm{Br}$. & \\
\hline 1900.628 & (B and $E$ ). & & & & 167.8 & 68.40 & 4,8 & 3 & 26-inch & 600 & See & \\
\hline 1900. 628 & (B and D). & & & & $247 \cdot 4$ & 69.28 & 4,8 & 3 & 26-inch & 600 & See & \\
\hline $1898.6,6$ & $\beta 636(A$ and $B)$ & 18 & 35 & +2 I 2 & 126.0 & 4. 65 & 4,4 & 2 & 26-inch & 600 & $\mathrm{Br}$. & \\
\hline $\begin{array}{r}1897.616 \\
.619 \\
.633 \\
\end{array}$ & $\Sigma 2283$. & 18 & 441 & +68 & $\begin{array}{l}86.0 \\
78.9 \\
82.7 \\
\end{array}$ & $\begin{array}{l}\text { I. } 07 \\
0.92 \\
1.03 \\
\end{array}$ & $\begin{array}{l}4,4 \\
6,4 \\
4,4\end{array}$ & $\begin{array}{c}\mathrm{I}-2 \\
2 \\
2\end{array}$ & \begin{tabular}{|cc}
$26-i n c h$ \\
"." \\
"“
\end{tabular} & $\begin{array}{l}600 \\
600 \\
600\end{array}$ & $\begin{array}{l}\mathrm{Br} . \\
\mathrm{Br} . \\
\mathrm{Br} .\end{array}$ & \\
\hline 1897.623 & & & & & 82.5 & I. or & & & & & & \\
\hline $\begin{array}{r}1903.604 \\
.629 \\
.634 \\
.637 \\
\end{array}$ & $\beta$ I32, $\ldots \ldots \ldots$ & 18 & $5 \quad 19$ & $|-1952|$ & \begin{tabular}{l|}
220.3 \\
223.4 \\
222.2 \\
$22 \mathrm{I} .2$ \\
\end{tabular} & \begin{tabular}{l|} 
o. 99 \\
I. 18 \\
o. 96 \\
I. 25 \\
\end{tabular} & $\begin{array}{l}4,6 \\
4,6 \\
4,6 \\
4,6\end{array}$ & $\begin{array}{l}f \\
f \\
b \\
b\end{array}$ & 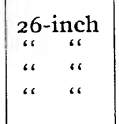 & $\begin{array}{l}600 \\
\ldots \\
600 \\
\cdots\end{array}$ & $\begin{array}{l}\text { D. } \\
\text { D. } \\
\text { D. } \\
\text { D. }\end{array}$ & $\begin{array}{l}\text { V e ry difficult; } \\
\text { blue, yellow. } \\
\text { Mags.equal; blue, } \\
\text { red. }\end{array}$ \\
\hline 1903.626 & & & & & 221.8 & I. 10 & & & & & & \\
\hline 1898.670 & $\beta 638(\mathrm{~B}$ and $\mathrm{C}) \ldots$ & 18 & 520 & +234 & 6.5 & I. 75 & 4,4 & 2 & 26-inch & 600 & $\mathrm{Br}$ & \\
\hline 1898.670 & (A and $B$ ). & & & & $15 x .8$ & $22.4 \mathrm{I}$ & 4,4 & 2 & 26-inch & 600 & $\mathrm{Br}$. & \\
\hline 1903.640 & $\mathrm{Hu}_{3} \mathbf{1}_{7} \ldots \ldots \ldots \ldots$ & I 8 & 654 & $+17 \times 2$ & 20.3 & I. 98 & 4,6 & $\mathrm{p}$ & 26-inch & $\cdots$ & D. & \\
\hline $\begin{array}{r}1903.593 \\
.604 \\
.610 \\
.629 \\
\end{array}$ & $\beta$ I3I $(A$ and $B)$ & 18 & $75 \mathrm{I}$ & -1537 & $\begin{array}{l}279.0 \\
279.1 \\
279.1 \\
279.0 \\
\end{array}$ & \begin{tabular}{l|}
2.83 \\
2.75 \\
2.92 \\
2.86
\end{tabular} & $\begin{array}{l}4,6 \\
4,6 \\
4,6 \\
4,6\end{array}$ & $\begin{array}{l}\mathrm{b} \\
\mathrm{g} \\
\mathrm{p} \\
\mathrm{g}\end{array}$ & $\begin{array}{cc}26-i n c h \\
\text { "، } \\
\text { “" } \\
\text { “" }\end{array}$ & $\begin{array}{l}600 \\
600 \\
600 \\
\cdots\end{array}$ & $\begin{array}{l}\text { D. } \\
\text { D. } \\
\text { D. } \\
\text { D. }\end{array}$ & $\begin{array}{l}\text { M a gs. } 7.7-9.2 \\
\text { yellow, red } \\
\text { Unsteady. }\end{array}$ \\
\hline 1903.609 & & & & & 279.0 & 2.84 & & & & & & . \\
\hline $\begin{array}{r}1903.593 \\
.604 \\
.629 \\
\end{array}$ & $(A$ and $C) .$. & & & & $\begin{array}{l}283.2 \\
284.7 \\
283.2 \\
\end{array}$ & $\begin{array}{l}7.13 \\
7.66 \\
7.5^{2} \\
\end{array}$ & $\begin{array}{l}4,6 \\
4,7 \\
4,6\end{array}$ & $\begin{array}{l}\mathrm{b} \\
\mathrm{g} \\
\mathrm{g}\end{array}$ & $\mid \begin{array}{c}26-\text { inch } \\
\text { ". } \\
\text { “ }\end{array}$ & $\begin{array}{l}600 \\
600 \\
\cdots\end{array}$ & $\begin{array}{l}\text { D. } \\
\text { D. } \\
\text { D. }\end{array}$ & $\begin{array}{l}\text { Mag. of C ro.4. } \\
\text { Mag. of C ro.2. }\end{array}$ \\
\hline 1903.609 & & & & & 283.7 & $7 \cdot 44$ & & & & & & \\
\hline 1897.619 & $0 \Sigma 345$ & I 8 & 755 & $+54^{8}$ & 62.9 & I. 18 & 4,4 & 2 & 26-inch & 600 & $\mathrm{Br}$ & $\begin{array}{l}\text { Unsteady and dif- } \\
\text { fuse. }\end{array}$ \\
\hline $\begin{array}{r}\mathrm{r} 903.593 \\
.604 \\
.629 \\
.634 \\
\end{array}$ & $\mathrm{Hn} \times 42 \ldots \ldots$ & 18 & 95 & $-11 \quad x 6$ & $\begin{array}{l}248.6 \\
247.8 \\
248.4 \\
249.8 \\
\end{array}$ & $\begin{array}{l}\text { I. } 10 \\
0.88 \\
0.85 \\
\text { I. } 05 \\
\end{array}$ & $\begin{array}{l}4,6 \\
4,6 \\
4,6 \\
4,6\end{array}$ & $\begin{array}{l}\mathrm{b} \\
\mathrm{g} \\
\mathrm{f} \\
\mathrm{b}\end{array}$ & 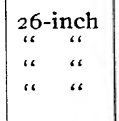 & $\begin{array}{l}600 \\
600 \\
600\end{array}$ & $\begin{array}{l}\text { D. } \\
\text { D. } \\
\text { D. } \\
\text { D. }\end{array}$ & Clouds. \\
\hline 1903.615 & & & & & 248.6 & 0.97 & & & & & & \\
\hline $\begin{array}{r}1903.604 \\
.610 \\
.629 \\
.634 \\
\end{array}$ & $\Sigma 2296 \ldots \ldots$ & r8 1 & 10 27 & -323 & $\begin{array}{l}8.1 \\
8.0 \\
7.4 \\
7.7 \\
\end{array}$ & \begin{tabular}{l|} 
3. 14 \\
3. 08 \\
3. 45 \\
3. 25 \\
\end{tabular} & $\begin{array}{l}4,6 \\
4,6 \\
4,6 \\
4,6\end{array}$ & $\begin{array}{l}\mathrm{g} \\
\mathrm{b} \\
\mathrm{f} \\
\mathrm{b}\end{array}$ & 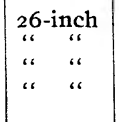 & $\begin{array}{l}600 \\
600 \\
\ldots \\
600\end{array}$ & $\begin{array}{l}\text { D. } \\
\text { D. } \\
\text { D. } \\
\text { D. }\end{array}$ & \\
\hline 1903.619 & & & & & $7 \cdot 8$ & 3. 23 & & & & & & \\
\hline
\end{tabular}




\begin{tabular}{|c|c|c|c|c|c|c|c|c|c|c|c|}
\hline \multirow{2}{*}{ 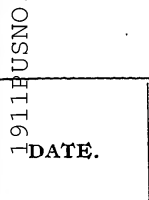 } & \multicolumn{11}{|c|}{ DOUBLE } \\
\hline & NAME. & $\begin{array}{l}\text { RIGHT } \\
\text { ASCENSION } \\
\text { I900.0 }\end{array}$ & $\begin{array}{l}\text { DECLI- } \\
\text { NATION } \\
\text { I9OO.O }\end{array}$ & $\begin{array}{c}\text { POSITION } \\
\text { ANGLE. }\end{array}$ & $\begin{array}{c}\text { DIS- } \\
\text { TANCE. }\end{array}$ & $\mid \begin{array}{c}\text { NUMBER } \\
\text { OF } \\
\text { MEASURES. }\end{array}$ & SEEING. & \begin{tabular}{|} 
INSTRU- \\
MENT.
\end{tabular} & POWER. & OBS'R. & REMARKS. \\
\hline $\begin{array}{r}1903.634 \\
.637 \\
\end{array}$ & $\beta_{463} \ldots \ldots$ & $\begin{array}{rrr}\mathrm{h} & \mathrm{m} & \mathrm{s} \\
\mathrm{I} 8 & \mathrm{II} & 53\end{array}$ & $\begin{array}{rr}0 & 1 \\
-16 & 54\end{array}$ & $\begin{array}{c}\circ \\
\text { IOI. } 4 \\
\text { IOI. } 6 \\
\end{array}$ & \begin{tabular}{l|}
$\prime \prime$ \\
1.86 \\
2.07
\end{tabular} & $\begin{array}{l}4,6 \\
4,6\end{array}$ & $\begin{array}{l}b \\
b\end{array}$ & "26-inch & $\begin{array}{l}600 \\
\cdots\end{array}$ & $\begin{array}{l}\text { D. } \\
\text { D. }\end{array}$ & Mags. 9.9-10.3. \\
\hline 1903.636 & & & & 101.5 & I. 96 & & & & & & \\
\hline $\begin{array}{r}1903.634 \\
.637 \\
.640 \\
.642\end{array}$ & Schj. I6 . . . . . . & $18 \quad 14 \quad 28$ & $-\begin{array}{ll}5 & 0\end{array}$ & $\begin{array}{l}196.5 \\
198.2 \\
197.5 \\
197.4\end{array}$ & $\begin{array}{l}3.03 \\
2.70 \\
2.72 \\
2.47\end{array}$ & $\begin{array}{l}4,6 \\
4,6 \\
4,6 \\
4,6\end{array}$ & $\begin{array}{l}b \\
p \\
p \\
f\end{array}$ & 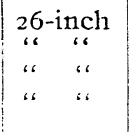 & $\begin{array}{l}600 \\
\cdots \\
\cdots \\
\cdots\end{array}$ & $\begin{array}{l}\text { D. } \\
\text { D. } \\
\text { D. } \\
\text { D. }\end{array}$ & \\
\hline 1903.638 & & & & $197 \cdot 4$ & 2.73 & & & & . & & \\
\hline $\begin{array}{r}\mathrm{I} 897.674 \\
\mathrm{I} 900.650 \\
.658 \\
\mathrm{I} 903.637 \\
.642 \\
.645\end{array}$ & $\Sigma 2303 \ldots \ldots \ldots \ldots \ldots$ & I $8 \quad 1439$ & $-8 \mathrm{I}$ & $\begin{array}{l}226.9 \\
229.2 \\
233.5 \\
225.8 \\
225.4 \\
222.5\end{array}$ & $\begin{array}{l}2.46 \\
2.45 \\
2.38 \\
2.63 \\
2.60 \\
2.64\end{array}$ & $\begin{array}{l}4,4 \\
4,8 \\
4,8 \\
4,6 \\
4,6 \\
4,6\end{array}$ & $\begin{array}{l}2 \\
\cdots \\
\cdots \\
\mathrm{p} \\
\mathrm{f} \\
\mathrm{f}\end{array}$ & 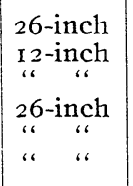 & $\begin{array}{l}600 \\
\ldots \\
\ldots \\
\ldots \\
\ldots \\
\ldots\end{array}$ & $\begin{array}{l}\mathrm{Br} . \\
\mathrm{B} . \\
\mathrm{B} . \\
\mathrm{D} . \\
\mathrm{D} . \\
\mathrm{D} .\end{array}$ & \\
\hline $\begin{array}{l}1897.674 \\
1900.654 \\
1903.641\end{array}$ & & & & $\begin{array}{l}226.9 \\
231.4 \\
224.6\end{array}$ & $\begin{array}{l}2.46 \\
2.42 \\
2.62\end{array}$ & & & & & & \\
\hline $\begin{array}{r}1903.637 \\
.642 \\
.645 \\
\end{array}$ & $\beta_{48} \ldots$ & $18 \quad 156$ & -1942 & $\begin{array}{l}359.9 \\
358.4 \\
360.6\end{array}$ & $\begin{array}{l}2.56 \\
2.34 \\
2.26\end{array}$ & $\begin{array}{l}4,6 \\
4,6 \\
4,6\end{array}$ & $\begin{array}{l}\mathrm{p} \\
\mathrm{p} \\
\mathrm{b}\end{array}$ & $\begin{array}{l}26-\text { inch } \\
" ، " ، \\
" ، " ،\end{array}$ & $\begin{array}{l}\cdots \\
\cdots \\
\cdots\end{array}$ & $\begin{array}{l}\text { D. } \\
\text { D. } \\
\text { D. }\end{array}$ & $\begin{array}{l}\text { Mags. } 8.6-9.5 . \\
\text { Mag s. } 8.2-9.2 \\
\text { yellow, red. }\end{array}$ \\
\hline $\mathrm{I} 903.64 \mathrm{I}$ & & & & 359.6 & 2. 39 & & & & & & \\
\hline I 897.655 & $\Sigma 2312 \ldots \ldots \ldots \ldots$ & $\begin{array}{lll}18 & \text { I } 7 & 14\end{array}$ & $+28 \mathrm{I7}$ & $\begin{array}{c}33^{8.9} \\
-\end{array}$ & I. 43 & 4,4 & 2 & 26-inch & 400 & $\mathrm{Br}$. & $\begin{array}{l}\text { Images unsteady } \\
\text { and diffuse. }\end{array}$ \\
\hline $\begin{array}{r}1903.593 \\
.637 \\
.642 \\
.645 \\
\end{array}$ & $\Sigma 2313 \ldots \ldots$ & 181921 & -639 & $\begin{array}{l}196.8 \\
197.3 \\
197.2 \\
197.5 \\
\end{array}$ & $\begin{array}{l}\text { 6. } 22 \\
6.11 \\
6.11 \\
6.12\end{array}$ & $\begin{array}{l}4,7 \\
4,6 \\
4,6 \\
4,6\end{array}$ & $\begin{array}{l}\mathrm{b} \\
\mathrm{p} \\
\mathrm{p} \\
\mathrm{f}\end{array}$ & \begin{tabular}{cc|}
$26-i n c h$ \\
$، "$ & 6 \\
$،$ & $،$ \\
$،$ & $،$
\end{tabular} & $\begin{array}{l}600 \\
\cdots \\
\cdots \\
\cdots\end{array}$ & $\begin{array}{l}\text { D. } \\
\text { D. } \\
\text { D. } \\
\text { D. }\end{array}$ & \\
\hline 1903.629 & & & & I97. 2 & 6. 14 & & & & & & \\
\hline I 897.693 & $0 \Sigma 350 \ldots \ldots$ & I8 220 & +622 & 167.2 & I. $8 \mathrm{I}$ & 4,4 & 2 & 26-inch & 600 & $\mathrm{Br}$. & \\
\hline $\begin{array}{r}1900.573 \\
.658 \\
.683\end{array}$ & $\Sigma 2316=59$ Serpentis. . & 18226 & to 8 & $\begin{array}{l}312.9 \\
321.5 \\
317.6\end{array}$ & $\begin{array}{l}\text { 4. } 26 \\
3 \cdot 71 \\
3.87\end{array}$ & $\begin{array}{l}4,8 \\
4,8 \\
4,8\end{array}$ & $\begin{array}{l}. \\
\cdots \\
\cdots\end{array}$ & $\begin{array}{c}\text { I2-inch } \\
" ، " ، \\
" ،\end{array}$ & $\begin{array}{l}\cdots \\
\cdots \\
\cdots\end{array}$ & $\begin{array}{l}\text { B. } \\
\text { B. } \\
\text { B. }\end{array}$ & Mags. 6.o-8.o. \\
\hline I900. 638 & & & 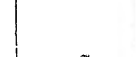 & $317 \cdot 3$ & $3 \cdot 95$ & & & & & & \\
\hline 1903.645 & $\beta 419 \ldots \ldots \ldots \ldots$ & I8 2647 & -754 & $49 \cdot 2$ & I. 17 & 4,6 & $\mathrm{~b}$ & 26-inch & $\cdots$ & D. & Yellow, orange. \\
\hline 1901.523 & $\Sigma 2345 \ldots \ldots \ldots \ldots \ldots$ & $\begin{array}{lll}18 & 31 & 14\end{array}$ & $+2 \mathrm{I} \quad 0$ & 205.2 & 8. 96 & 4,8 & $2-3$ & 26-inch & 400 & La. & \\
\hline 1897.693 & $0 \Sigma 359 \ldots \ldots \ldots \ldots \ldots$ & $18312 I$ & +2332 & 341.6 & o. 39 & 4,4 & 3 & 26-inch & 888 & $\mathrm{Br}$. & \\
\hline $\begin{array}{r}1903.637 \\
.642 \\
\end{array}$ & $\Sigma 2347=196$ Serpentis $\ldots$ & 183248 & -028 & $\begin{array}{l}257.8 \\
258.2 \\
\end{array}$ & $\begin{array}{l}3.20 \\
3 \cdot 38 \\
\end{array}$ & $\begin{array}{l}4,6 \\
4,6\end{array}$ & $\begin{array}{l}\mathrm{p} \\
\mathrm{p}\end{array}$ & 26-inch & $\begin{array}{l}\cdots \\
\cdots\end{array}$ & $\begin{array}{l}\text { D. } \\
\text { D. }\end{array}$ & Yellow, red. \\
\hline 1903.640 & & & & 258.0 & 3.29 & & & & & & \\
\hline
\end{tabular}

$28167^{\circ}-$ VOL $6-\mathrm{II}-\mathrm{II}$ 


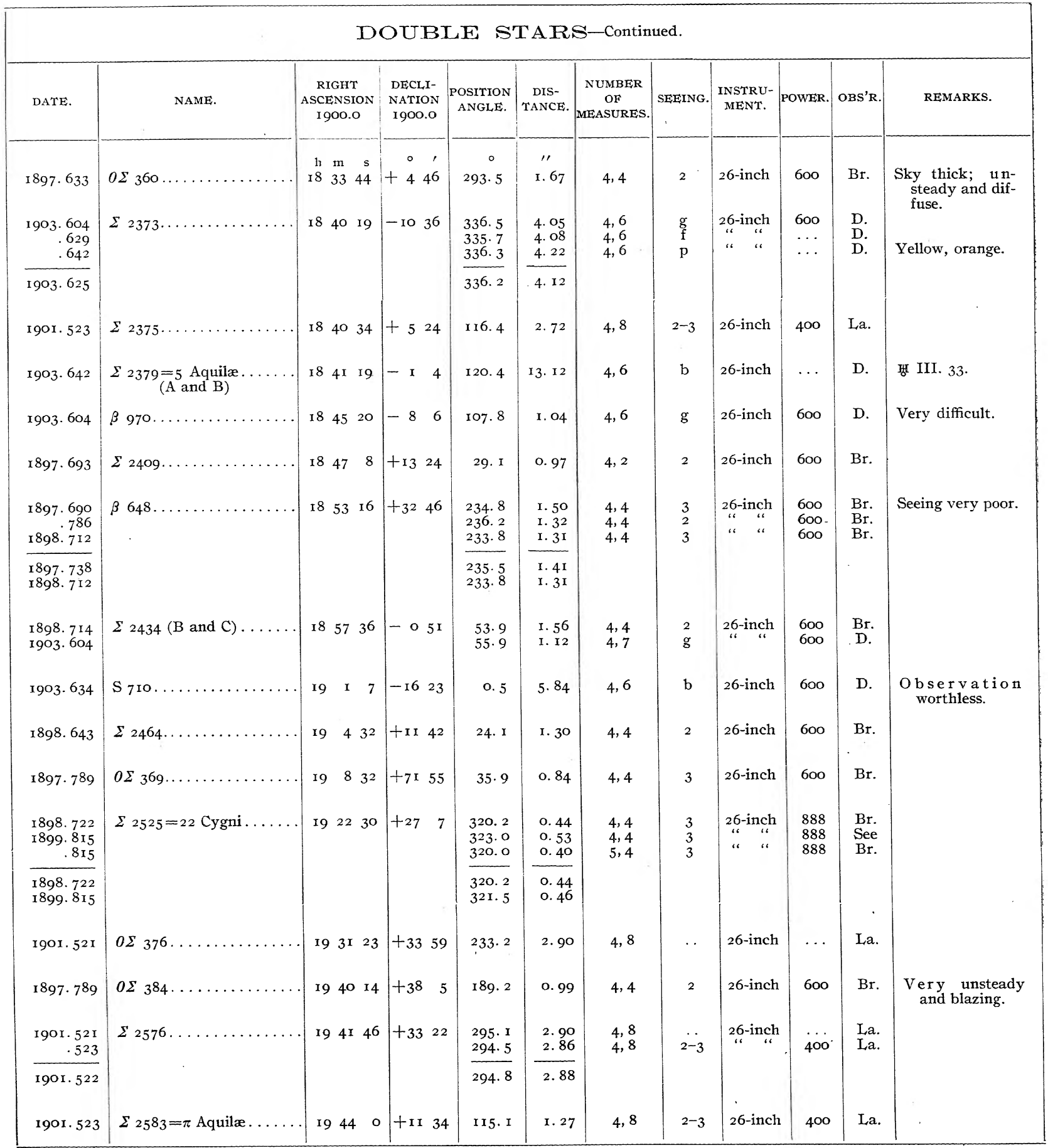




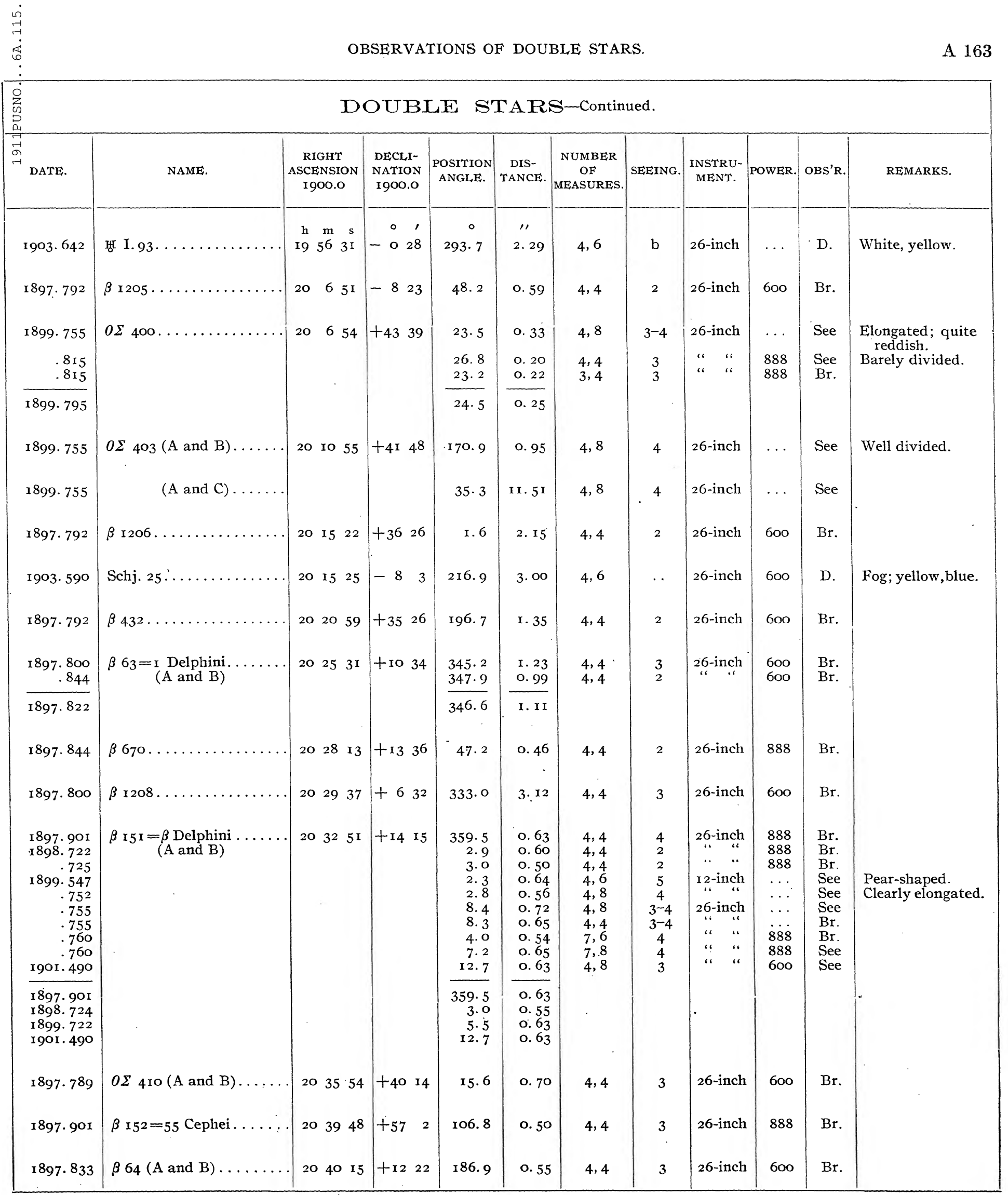




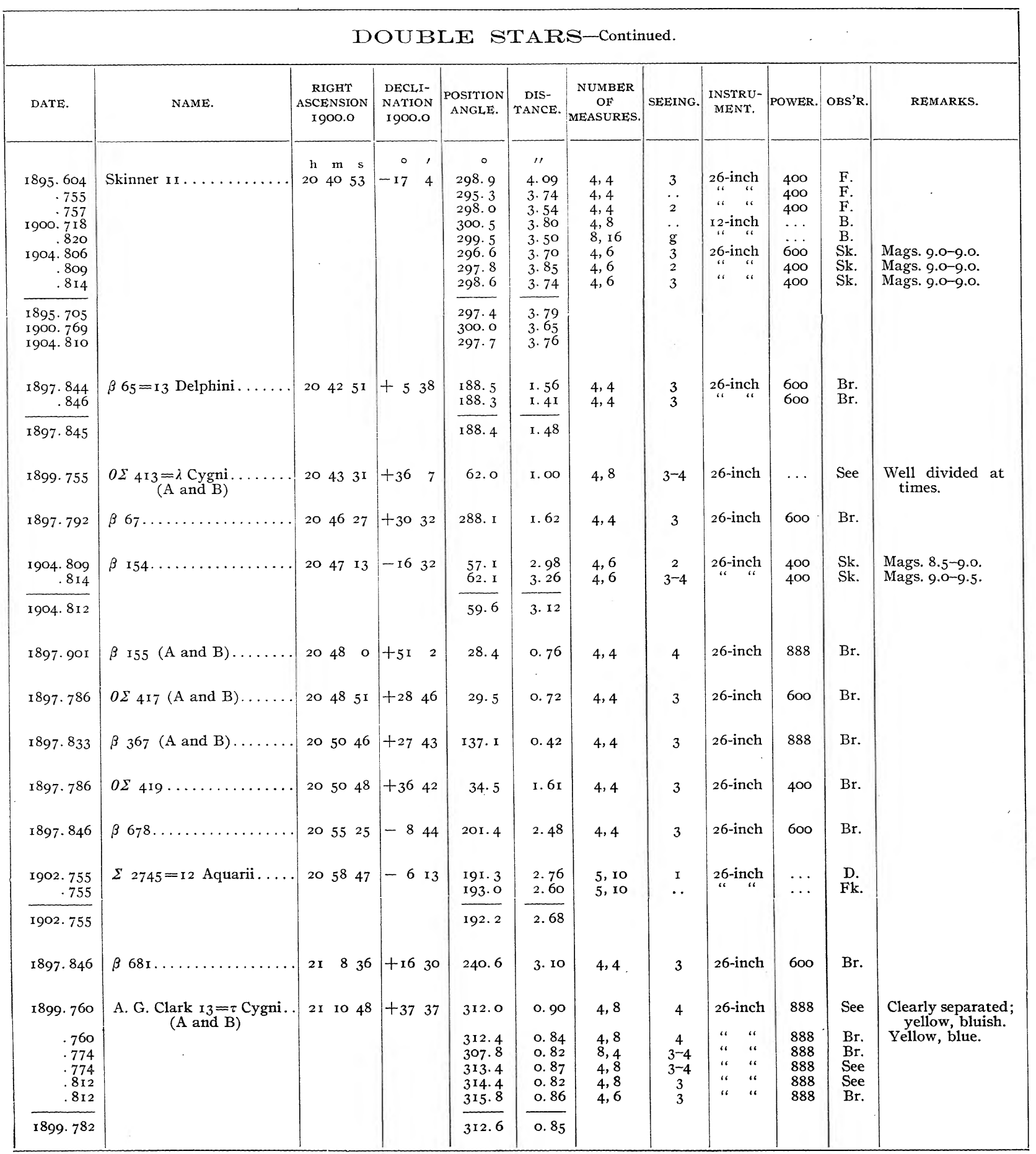


DOUBIE STARS-Continued.

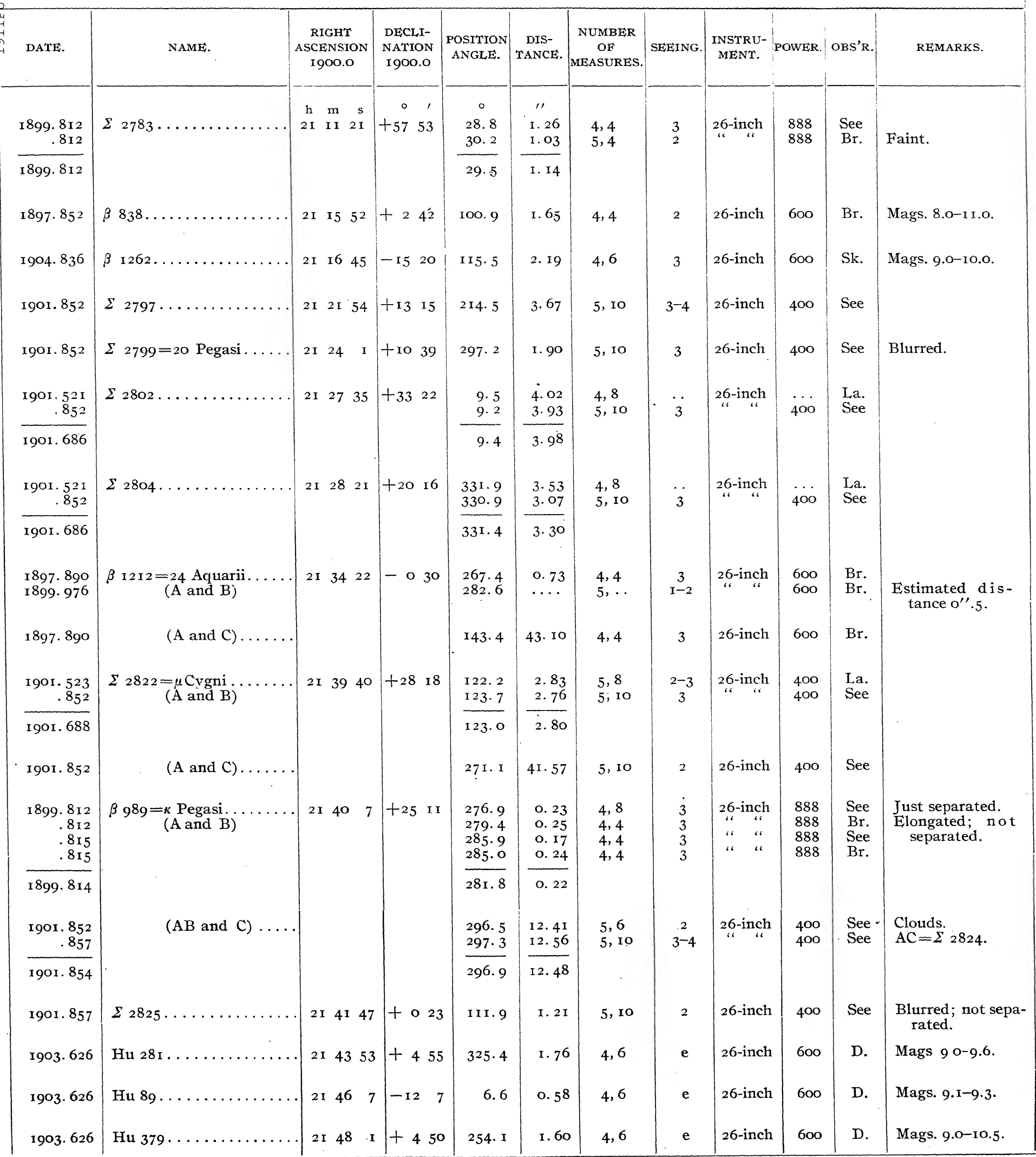




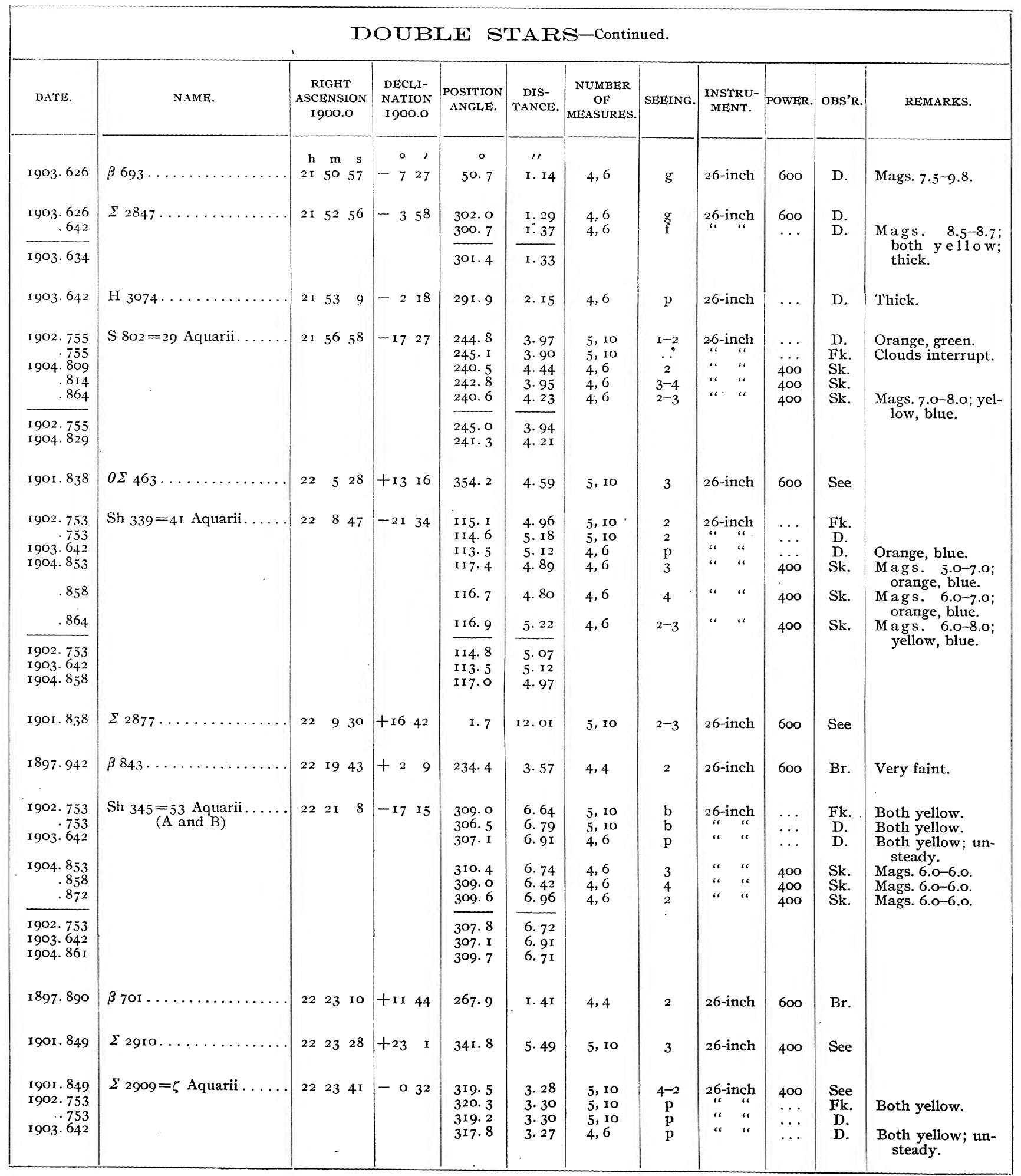




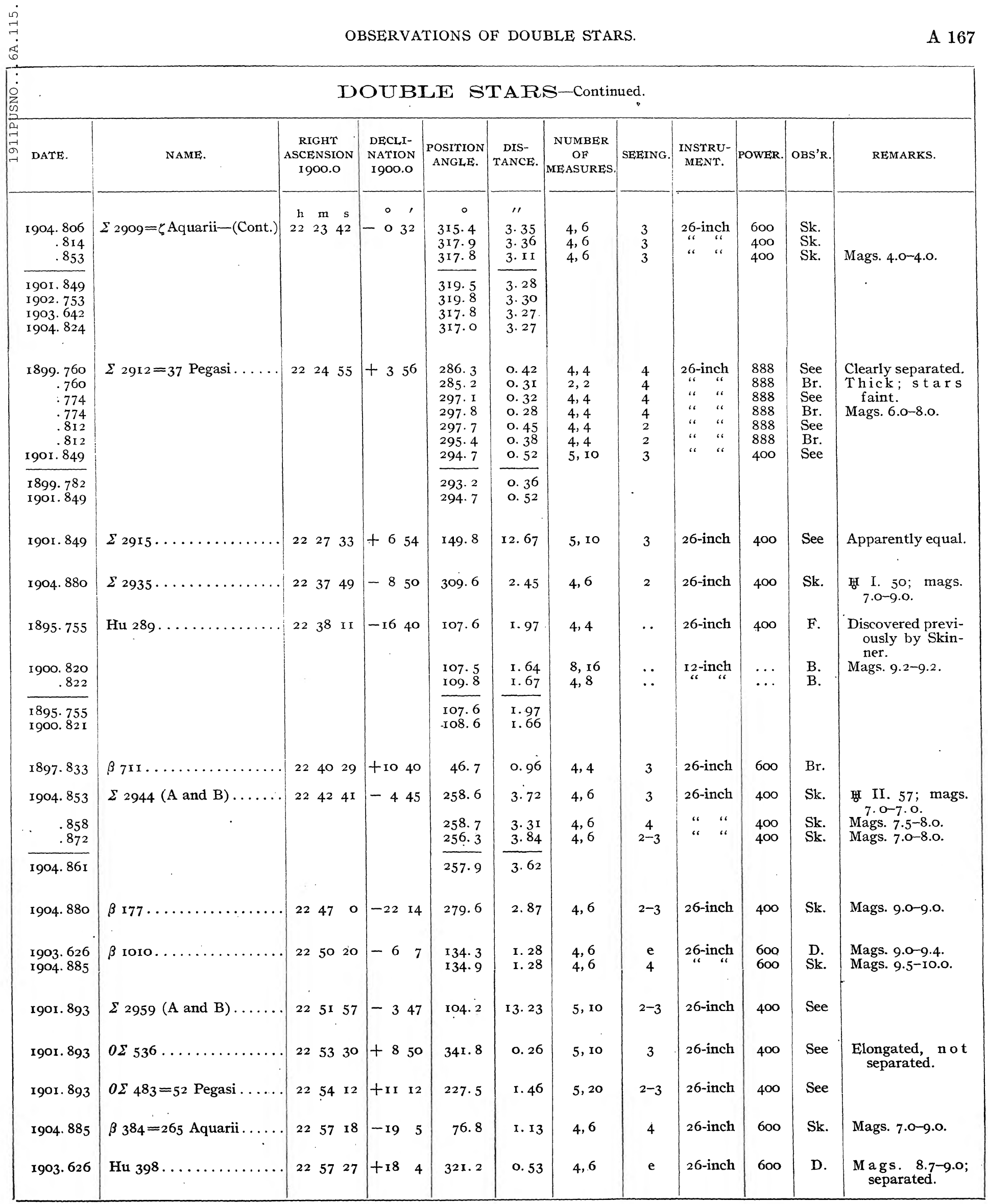




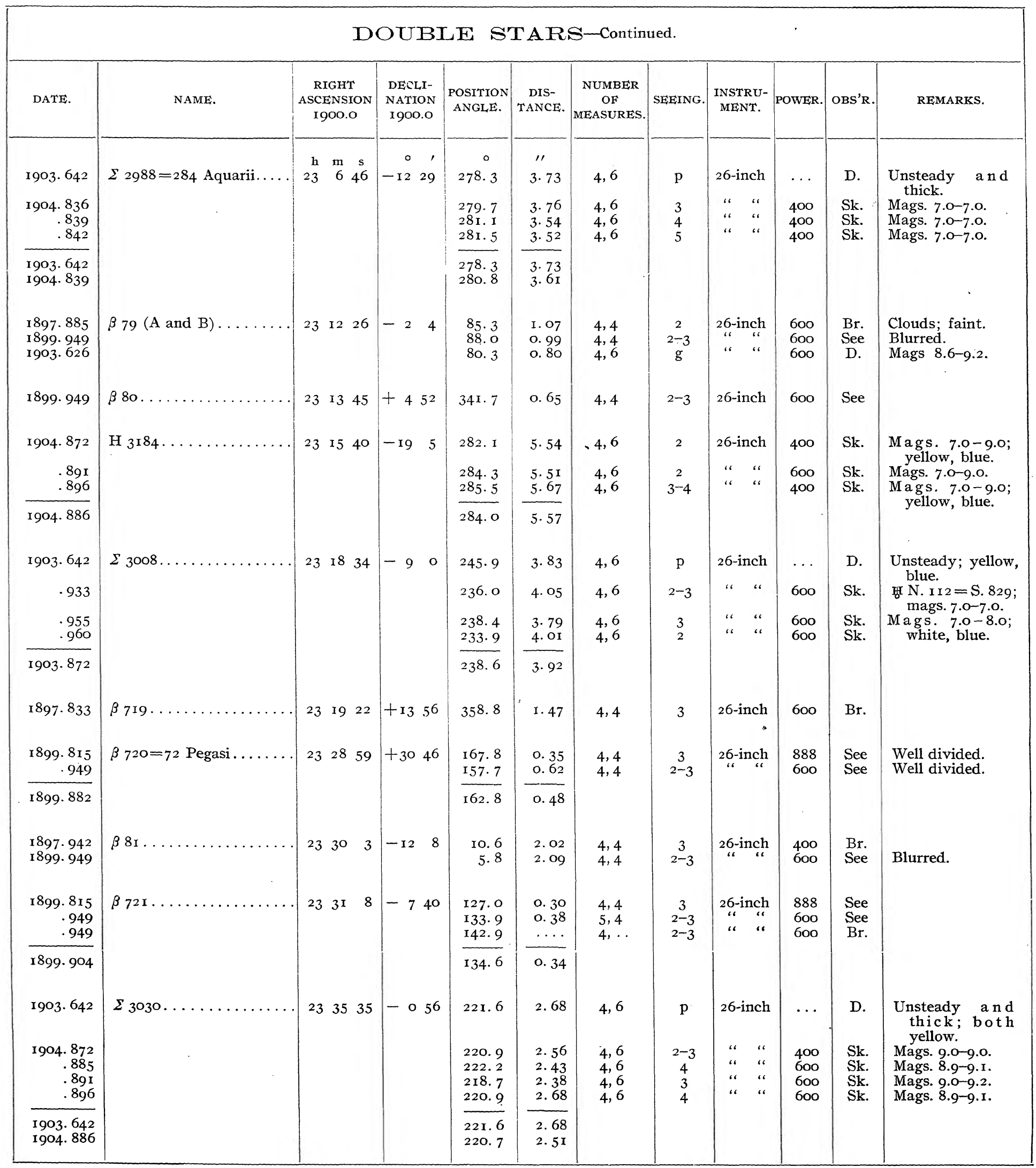


DOUBLE STARS-Continued.

\begin{tabular}{|c|c|c|c|c|c|c|c|c|c|c|c|}
\hline $\begin{array}{l}\text { م } \\
\text { 검 } \\
\text { DATE. }\end{array}$ & NAME. & $\begin{array}{l}\text { RIGHT } \\
\text { ASCENSION } \\
\text { I9OO.O }\end{array}$ & $\begin{array}{l}\text { DECLI- } \\
\text { NATION } \\
\text { I9O0.0 }\end{array}$ & $\begin{array}{c}\text { POSITION } \\
\text { ANGLE. }\end{array}$ & $\begin{array}{l}\text { DIS- } \\
\text { TANCE. }\end{array}$ & $\begin{array}{c}\text { NUMBER } \\
\text { OF } \\
\text { MEASURES. }\end{array}$ & SEEING. & $\begin{array}{l}\text { INSTRU- } \\
\text { MENT. }\end{array}$ & POWER. & OBS'R. & REMARKS. \\
\hline I 899.949 & $\beta$ 1223 $\ldots \ldots \ldots \ldots \ldots$ & $\begin{array}{rrr}\mathrm{h} & \mathrm{m} & \mathrm{s} \\
23 & 40 & \mathrm{I} 2\end{array}$ & $\begin{array}{r}01 \\
+\quad 434\end{array}$ & $\begin{array}{c}\circ \\
293 \cdot 9\end{array}$ & $\begin{array}{l}\prime \prime \\
\text { I. } 44\end{array}$ & 4,4 & 2 & 26-inch & 600 & See & \\
\hline I903. 626 & $\mathrm{Hu} 300 \ldots \ldots \ldots \ldots$ & $2340 \cdot 24$ & $+55^{6}$ & I $22 . \mathrm{I}$ & I. I 5 & 4,6 & $\mathrm{e}$ & 26-inch & 600 & D. & Mags. 8.9-9.0. \\
\hline $\begin{array}{r}1904.839 \\
.842 \\
.858\end{array}$ & Sh $356=107$ Aquarii. . . . & 234049 & $-19 \quad 14$ & $\begin{array}{l}\text { I38. } 2 \\
\text { 137. } 7 \\
\text { 140. } 2\end{array}$ & $\begin{array}{l}5 \cdot 7^{8} \\
6.15 \\
5 \cdot 97\end{array}$ & $\begin{array}{l}4,6 \\
4,6 \\
4,6\end{array}$ & $\begin{array}{l}4 \\
4\end{array}$ & $\begin{array}{l}\text { 26-inch } \\
\text { "، } ، \\
\text { " }\end{array}$ & $\begin{array}{l}400 \\
400\end{array}$ & $\begin{array}{l}\text { Sk. } \\
\text { Sk. } \\
\text { Sk. }\end{array}$ & $\begin{array}{l}\text { HI II. } 24 ; \text { mags. } \\
5.0-7.0 ; \text { sky } \\
\text { very thick. } \\
\text { Mags. 6.0-7.0. } \\
\text { Mags. 6.0-7.0. }\end{array}$ \\
\hline I904. 846 & & & & 138.7 & $5 \cdot 97$ & & & & & & \\
\hline I904. $89 \mathrm{I}$ & $\Sigma 3036 \ldots \ldots \ldots \ldots$ & 234053 & - 0 I 8 & $2,23 \cdot 2$ & 2.82 & 4,6 & $2-3$ & 26-inch & 600 & Sk. & $\begin{array}{l}\text { Mags. } 8.0-1 \text { i.o; } \\
\text { straw, blue. }\end{array}$ \\
\hline I899. 815 & $\beta 726 \ldots \ldots \ldots \ldots \ldots$ & $2341 \quad 25$ & $-13 \quad 19$ & 318.0 & o. 75 & 4,4 & 3 & 26-inch & 600 & See & \\
\hline $\begin{array}{r}1903.955 \\
.960 \\
1904.891\end{array}$ & $\Sigma 3046 \ldots \ldots \ldots \ldots$ & 235 I I 5 & -103 & $\begin{array}{l}249 \cdot 8 \\
247 \cdot 4 \\
248 \cdot 9 \\
\end{array}$ & $\begin{array}{l}\text { 3. } 19 \\
\text { 3. } 18 \\
\text { 3. } 16 \\
\end{array}$ & $\begin{array}{l}4,6 \\
4,6 \\
4,6\end{array}$ & $\begin{array}{l}2 \\
2 \\
3\end{array}$ & $\begin{array}{cc}26 \text {-inch } \\
" ، \\
" 1\end{array}$ & $\begin{array}{l}600 \\
600 \\
600\end{array}$ & $\begin{array}{l}\text { Sk. } \\
\text { Sk. } \\
\text { Sk. }\end{array}$ & $\begin{array}{l}\text { Mags. 8.o-8.o. } \\
\text { Very unsteady. } \\
\text { Mags. 8.8-9.0. }\end{array}$ \\
\hline $\begin{array}{l}1903.958 \\
1904.891\end{array}$ & - & & & $\begin{array}{l}248.6 \\
248.9\end{array}$ & $\begin{array}{l}\text { 3. } 18 \\
\text { 3. } 16\end{array}$ & & & & & & \\
\hline $\begin{array}{r}1902.917 \\
.917 \\
\end{array}$ & $\Sigma 3050=37$ Andromedæ. & 235423 & +33 xo & $\begin{array}{l}214 \cdot 5 \\
214 \cdot 5 \\
\end{array}$ & $\begin{array}{l}2.68 \\
2.66\end{array}$ & $\begin{array}{l}5, \text { Io } \\
5, \text { Io }\end{array}$ & $\begin{array}{l}2 \\
3\end{array}$ & 26-inch & $\begin{array}{l}600 \\
600\end{array}$ & $\begin{array}{l}\text { Sk. } \\
\text { Fk. }\end{array}$ & \\
\hline 1902.917 & & & & $2 \mathrm{I} 4 \cdot 5$ & 2.67 & & & & & & \\
\hline $\begin{array}{r}1903.626 \\
1904.891 \\
.896\end{array}$ & $\beta 731 \ldots \ldots \ldots \ldots \ldots$ & 235429 & -822 & $\begin{array}{l}266.3 \\
265.0 \\
267.8 \\
266.3 \\
266.4\end{array}$ & $\begin{array}{l}\text { I. } 70 \\
\text { I. } 53 \\
\text { I. } 75 \\
\text { I. } 70 \\
\text { I. } 64\end{array}$ & $\begin{array}{l}4,6 \\
4,6 \\
4,6\end{array}$ & $\begin{array}{c}\mathrm{e} \\
2-3 \\
3\end{array}$ & $\begin{array}{c}26 \text {-inch } \\
\text { " } 64 \\
\end{array}$ & $\begin{array}{l}600 \\
600 \\
600\end{array}$ & $\begin{array}{l}\text { D. } \\
\text { Sk. } \\
\text { Sk. }\end{array}$ & $\begin{array}{l}\text { Mags. } 8.3-9 \cdot 4 \\
\text { Mags. } 8.5-9 \cdot 5 \\
\text { M ag s. } 8.5-9.5 \\
\text { thin c } 1 \text { oud } \\
\text { but steady. }\end{array}$ \\
\hline $\begin{array}{r}1899.785 \\
.815 \\
.815 \\
\end{array}$ & $\beta 733=85$ Pegasi. $\ldots \ldots \ldots$ & 235655 & +2634 & $\begin{array}{l}235 \cdot 8 \\
227 \cdot 2 \\
233 \cdot 4 \\
\end{array}$ & $\begin{array}{l}\text { o. } 82 \\
\text { o. } 81 \\
\text { o. } 86\end{array}$ & $\begin{array}{l}4,4 \\
4,4 \\
4,4\end{array}$ & $\begin{array}{l}3 \\
3 \\
3\end{array}$ & $\begin{array}{c}26-\mathrm{inch} \\
6 " 6 \\
،\end{array}$ & $\begin{array}{l}888 \\
600 \\
600\end{array}$ & $\begin{array}{l}\text { See } \\
\text { See } \\
\text { Br. }\end{array}$ & Reddish, blue. \\
\hline $\mathrm{r} 899.805$ & & & & 232.1 & o. 83 & & & & & & \\
\hline
\end{tabular}


